\title{
Holographic Data Storage Systems
}

\section{LAMBERTUS HESSELINK, SERGEI S. ORLOV, AND MATTHEW C. BASHAW}

\author{
Invited Paper
}

In this paper, we discuss fundamental issues underlying holographic data storage: grating formation, recording and readout of thick and thin holograms, multiplexing techniques, signal-to-noise ratio considerations, and readout techniques suitable for conventional, phase conjugate, and associative search data retrieval. Next, we consider holographic materials characteristics for digital data storage, followed by a discussion on photorefractive media, fixing techniques, and noise in photovoltaic and other media with a local response. Subsequently, we discuss photopolymer materials, followed by a discussion on system tradeoffs and a section on signal processing and en/decoding techniques, succeeded by a discussion on electronic implementations for control, signal encoding, and recovery. We proceed further by presenting significant demonstrations of digital holographic systems. We close by discussing the outlook for future holographic data storage systems and potential applications for which holographic data storage systems would be particularly suited.

Keywords-Diffraction gratings, gratings, high density data storage, holographic data storage, holographic recording materials, holography, optical data storage, optical storage materials, photopolymer media, photorefractive media, volumetric data storage.

\section{INTRODUCTION}

\section{A. Motivation}

Optical data storage is a commercial success story. Each year billions of recordable disks are sold worldwide, and in almost every household with a computer there is a CD-ROM or CD-recordable drive. The industry published roadmap shows future DVD products to reach capacities of near 100 $\mathrm{GB}$ on a disk, and data transfer rates exceeding $300 \mathrm{Mb} / \mathrm{s}$ sometime in the latter half of the current decade, shown in Fig. 1. Such high capacities are obtained by using a very high numerical aperture (NA) optical stylus for reading data

Manuscript received March 7, 2003; revised March 9, 2004. This work was primarily supported by the Defense Advanced Research Projects Agency Industry/University PRISM and HDSS Programs and, subsequent to 2000 , in part by the Japanese Science and Technology program.

L. Hesselink and S. S. Orlov are with the Solid State Photonics Lab, Department of Electrical Engineering, Stanford University, Palo Alto, CA 94305-4070 USA (e-mail: orlov@wireless.net).

M. C. Bashaw is with Lockheed Martin Corporation, Integrated Systems \& Solutions, San Jose, CA 95134 USA.

Digital Object Identifier 10.1109/JPROC.2004.831212

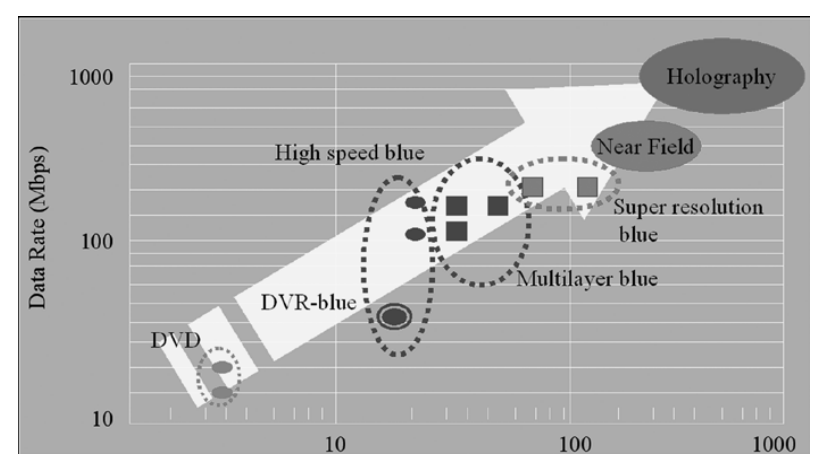

Fig. 1. Optical roadmap. (Courtesy TDK, with permission).

from and writing data onto the optical disk. Blue lasers achieve spot sizes of a few hundred micrometers, providing approximately $25 \mathrm{~GB}$ of storage capacity per layer. Up to four layers - two on each side-increase capacity to near 100 GB per disk. To further improve capacity and transfer rates, several options are available, including increasing the NA beyond 0.85 , reducing the wavelength below $400 \mathrm{~nm}$, or adding more layers. All these options present significant obstacles. Additional improvements in NA require more costly and complicated optical systems for an additional $40-50 \%$ gain in NA, leading to doubling in capacity. Shorter wavelength lasers are not commercially available and require special optical materials that are transparent below 400 nm. Increasing the number of layers also proves difficult for a variety of reasons, the most significant one being manufacturability and complexity of implementation for the media, optical stylus, and the associated optomechanical system for tracking and focusing. The industry, therefore, has been researching new methods for extending the optical data storage roadmap well beyond 100 GB per disk.

Two promising candidates are near-field recording and holography. In near-field recording approaches, the NA of the optical stylus is made larger than one, resulting in very small spot sizes approaching dimensions less than $100 \mathrm{~nm}$. The focusing spot is near to the optical lens, requiring close proximity between the optical head and disk, making removability of the media more difficult, as small contaminants 
can cause interference between the two. The advantages of near-field recording are the extremely high areal densities achievable, but at the expense of optomechanical complexity and less robust removability, among others. The second promising candidate technology is optical holography. In optical holography, data are stored throughout the volume of the recording medium, as opposed to on the surface. Data are impressed onto an optical coherent beam using a spatial light modulator (SLM) or page composer. The signal bearing beam is interfered with a reference beam inside the recording medium to produce an interference grating, representing a data page. Multiple gratings are superimposed by varying the optical properties of the reference beam, a process referred to as multiplexing. Upon data retrieval or readout, a single reference beam is incident on the medium under the same conditions as used for storage, producing a diffracted beam representing the stored data page. The diffracted beam is detected by a detector array, which allows extraction of the stored data bits from the measured intensity pattern. Data encoding and image processing techniques enhance data robustness. Data pages contain large numbers of data bits or pixels; practically up to 1 million $\mathrm{b} /$ page have been demonstrated. As a whole page is either stored or recalled by the reference beam, data transfer rates can be phenomenally high, exceeding $10 \mathrm{~Gb} / \mathrm{s}$. By superimposing multiple data pages in the same volume, data storage capacity is very high as well. Although not quite physically correct, we can think of the number of superimposed holograms as the equivalent number of layers in a multilayer surface recording system. Instead of the two or four layers used in conventional optical recording, holographic recording can support hundreds of superimposed holograms or "equivalent layers" by employing the third dimension of storage media. This leads to powerful features not available from surface recording technologies, but also specific problems that need to be addressed before holographic data storage can be a commercially viable option for the optical roadmap.

Among the unique advantages of holographic storage are extremely short access times, less than $50 \mu \mathrm{s}$, extremely fast input and output rates, exceeding $10 \mathrm{~Gb} / \mathrm{s}$, as well as enormous search capabilities for finding unindexed information in databases at rates exceeding $100 \mathrm{~Gb} / \mathrm{s}$, far superior to current magnetic disk based storage systems. These advantages result from storing data in a volume in the form of data pages containing hundreds of thousands of bits. An all-solid-state memory device could contain gigabytes of data, accessible in microseconds, and allowing searches of image features and recognition at unmatched rates. The powerful advantages of holographic storage have propelled decades of research and development of this technology. Ironically, the challenges facing commercialization of holographic storage technology also result from the same desirable holographic features. As many holograms are superimposed, media must exhibit excellent volumetric stability, low scatter, and high sensitivity for recording, and must be easily manufacturable. Key optical components such as the page composer and the readout detector array must be of high performance, i.e., contain large

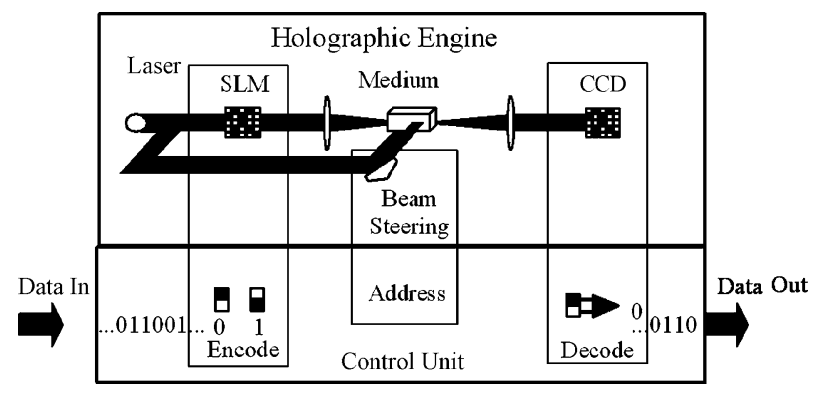

Fig. 2. Perpendicular $\left(90^{\circ}\right)$ angle multiplexing architecture, including the electronic control unit.

numbers of pixels and have fast frame rates, exceeding thousands of frames per second for pages containing hundreds of thousands of bits. The sensitivity of the detector must be high, as superposition of holograms reduces the intensity of the diffracted data pages as more and more pages are stored in the same volume. The larger the capacity, the smaller the readout signal, presenting a challenge to system performance optimization.

At present, it is uncertain which technology, near-field recording or holography, will be selected by the optical storage industry, sparking intense interest in both. According to IBM [1], holography, with its unique performance, may find that applications in all-solid-state devices have extremely short access times, effectively alleviating the large I/O gap that exists between solid-state memory and hard disk drive access times, which gives rise to latency that reduces system performance. Memories could compete in both the optical and magnetic storage markets. Holographic memories could also be employed in large data warehouses where the extreme search and readout rates facilitate more efficient database management of large data repositories and involving many users. Holography, it seems, is an attractive approach for future storage systems.

\section{B. Holographic Storage Architectures}

The holographic storage system architecture is largely determined by the type of recording medium. Broadly speaking, holographic data storage materials are divided into two classes; systems based on thin (a few hundred micrometers thick) photosensitive organic media and thick (a few millimeters to centimeters), inorganic photorefractive crystals [2]. Thick, bulk crystals of photorefractive media are ideal for recording geometries in which a reference and object beam are incident on the medium at right angles, as shown in Fig. 2. A typical photorefractive crystal used to investigate this configuration is iron-doped lithium niobate. A laser beam is split into two beams, a reference beam and an object beam. Data are imprinted on the object beam via a page composer or SLM. The reference and object beams are focused and combined on the recording medium. The medium is photosensitive and a phase hologram is recorded. By varying the reference beam angle, hundreds of holograms are superimposed in a single location of the medium. By scanning both beams over the medium, or by translating the 


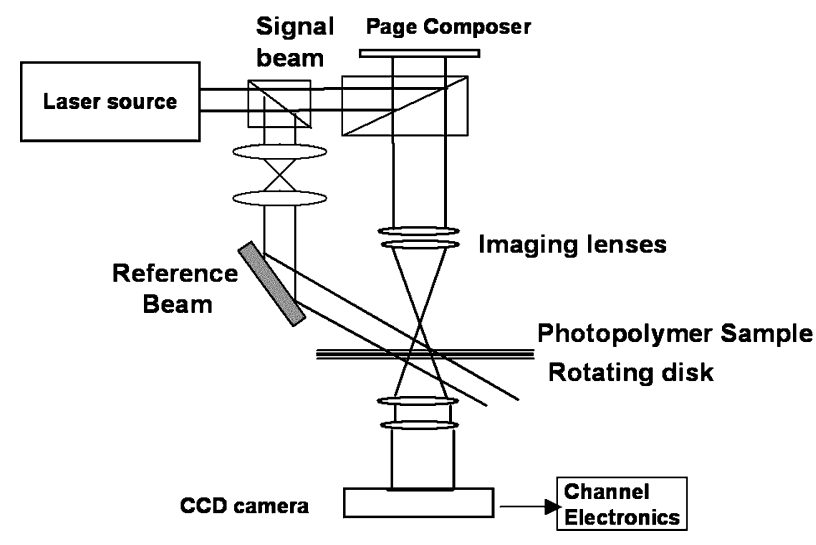

Fig. 3. Shift multiplexing architecture using rotating photopolymer disk medium.

medium relative to the optical beams, the total recording volume is utilized. Upon illumination with a single reference beam, the corresponding data page is retrieved at the detector array. Such systems excel in having ultrashort access times - on the order of a few tens of microseconds-and extremely fast data retrieval rates exceeding $10 \mathrm{~Gb} / \mathrm{s}$, as well as all-solid-state operation with no moving parts. The media are rewritable and information can be fixed for tens of years, if not centuries. Based on fundamental considerations, storage capacity is typically tens of gigabytes, and write times are slower than readout times by one to two orders of magnitude. Complete demonstration systems have been built by Stanford University, Stanford, CA, and Siros [3], IBM [4] and Rockwell, Thousand Oaks, CA [5], as part of the photorefractive information storage materials (PRISM) and holographic data storage systems (HDSS) programs; while researchers at the California Institute of Technology, Pasadena (Caltech) [6], and at Lucent [7] and at other organizations around the world have subsequently made system demonstrations as well. In particular, much work has been carried out at Caltech on optical architectures based on $\mathrm{LiNbO}_{3}$. Notably, the Psaltis group at Caltech and the group at Northrop have demonstrated analog recordings of large numbers of holograms [8].

Polymer-based systems resemble conventional DVD systems, having a rotating, removable thick $(0.5-1 \mathrm{~mm})$ holographic polymer disk, and a two-sided optical head, as shown in Fig. 3. An image bearing object and reference beam are incident on the recording medium. The medium is a photosentive polymer, typically sandwiched between two parallel glass plates with a thickness of a few hundred micrometers. Due to the small thickness of the medium, conventional angular and wavelength multiplexing do not allow hundreds of holograms to be superimposed [2], because eventually interpage crosstalk becomes too large. Upon illumination by light, a hologram is recorded through a chemical reaction that polymerizes an initially gel-like medium. By rotating the disk underneath both beams, a new hologram can be superimposed at a slightly shifted location, partially overlapping previously recorded holograms (shift multiplexing [9], [10]). Data are read out by illuminating the rotating disk with the reference beam, generating the stored data page and measuring it on the detector array. Shift multiplexing can be implemented by using a spherical wave [11] or, alternatively, a random or pseudorandom speckle pattern as a reference beam [12]. By modifying the phase front of the reference beam using a random or pseudorandom phase mask to generate a speckle pattern, higher shift selectivities can be achieved than in the case of a simple spherical wave. Alignment requirements, however, increase as well, as we need to maintain a constant phase relationship between the signal and reference beam.

In photopolymer media, shrinkage is a considerable issue, and tradeoffs are made to control shrinkage versus recording sensitivity and total dynamic range of the medium. Photopolymers, on the positive side, are more sensitive recording media by at least one or two orders of magnitude than photorefractive crystals. They derive their advantage from an exothermal chemical chain reaction that "amplifies" one photon event into 100 or 1000 chain polymerization events, whereas photorefractive media typically require one photon event for every photorefractive event that produces a local index of refraction change in the medium. Consequently, sensitive photopolymer materials are write-once-read-many (WORM) media. Attempts to make reversible polymer media with high sensitivity have fallen short of the requirements for HDSSs [13], as they fundamentally require again one photon event for one index changing event. The price to pay for higher sensitivity of photopolymers is the change in media density, which leads to both an index change as well as shrinkage caused by the polymerization reaction. The holy grail for developers of holographic data storage materials developers is to devise a medium with high sensitivity while maintaining a low level of shrinkage. Researchers at Polaroid, Boston, MA, and Bell Laboratories, Murray Hill, NJ, have taken different tacks on this problem, and excellent system demonstrations based on cationic ring opening materials (CROP) (Polaroid) [14], [15] and free-radial media (Lucent) have been made [16]. Data capacities exceeding $250 \mathrm{~GB}$ on a DVD-like disk have been measured by Aprilis, and data rates exceeding $10 \mathrm{~Gb} / \mathrm{s}$ have been demonstrated at Stanford University using Polaroid, and later Aprilis, CROP media [17], [18], To further increase data storage density or capacity per disk, additional material improvements and system optimization are needed.

System optimization, as usual, is a complex problem involving a large number of tradeoffs. For HDSSs in particular, the tradeoff between capacity and transfer rate is different from other storage systems. Fundamentally, holographic data storage is based on multiplexing many holograms in the same volume of the recording medium. For media with a linear response, this implies that the dynamic range for each hologram is roughly equal to the total dynamic range of the medium divided by $N$, the number of holograms. As the diffraction efficiency of each hologram is proportional to the squared index modulation, readout signal strength drops off as $1 / N^{2}$. The larger the capacity of the device is, the smaller the readout signal strength and the signal-to-noise ratio (SNR) are. In turn, small SNR causes large raw bit-error rate (BER), which above a threshold of $10^{-3}$ to $10^{-4}$ cannot be further lowered by error correction schemes. To boost 
the SNR, detector integration times can be increased at the expense of data transfer rates. High capacity systems are, therefore, easiest demonstrated for small transfer rates, and vice versa.

\section{Brief History and State of the Art}

In the early 1970s, photorefractive media were investigated for use in holographic data storage devices because of the potentially high storage capacity [19]-[21]. Theoretical investigations indicated that a potential storage density of $10^{13} \mathrm{~b} / \mathrm{cm}^{3}$ could be achieved [22], with data transfer rates exceeding gigabits per second. At that time, however, the necessary input and output devices were not available to take advantage of the high data bandwidths that page storage affords. It was also difficult to fix information in the crystals, resulting in data erasure upon readout. The full potential of holographic data storage was never realized.

In the mid-1980s, the Micro Computer Corporation (MCC, Austin, TX) and Stanford University started another effort to build a complete HDSS [23] based on the view that disk access latency - the time it takes to find information on a hard drive-was significantly impairing computer performance. This effort led to significant advances in components and systems, but the key limiting element in the system was the material. In 1994 and 1995, the Defense Advanced Research Projects Agency (DARPA) initiated two consortia, the PRISM and HDSS programs, to address key holographic issues, led by Stanford University as Principal Investigator (PI) (L. Hesselink), and IBM Almaden as Co-PI (G. Sincerbox, later succeeded by H. Coufal)). The PRISM activities were focused on fundamental recording material issues, while HDSS focused on research and development of new optical and electronic components and digital holographic storage systems. As a result of this team effort, Stanford University has developed, built, and tested a complete, very high transfer rate, and high capacity holographic disk storage system [14] including hardware implemented holographic channel decoding electronics for transfer rates exceeding $10 \mathrm{~Gb} / \mathrm{s}$, while providing high capacity storage ( $>100 \mathrm{~Gb}$ per 6.5 -in-diameter disk) in WORM photopolymer media. This system is, to the best of our knowledge, the only complete holographic system built to date; it also still has record-setting performance. More recently, Aprilis has demonstrated data densities exceeding $250 \mathrm{~b} / \mu \mathrm{m}^{2}$. Also, data search capabilities exceeding $20 \mathrm{~Gb} / \mathrm{s}$, much faster than magnetic disk drives have been reported.

The achieved performance came about as a result of significant improvements in the components, subsystems, and, most importantly, the recording materials. The PRISM and HDSS programs delivered significantly new materials, most noticeably polymer-based WORM disks that are two to three orders of magnitude more sensitive than the best inorganic photorefractive read-write crystals, new laser sources, very large charge-coupled device (CCD) detector arrays and SLM arrays, high-powered red lasers, as well as new digital encoding techniques and novel processes for system operation. By the end of 1999, the PRISM and HDSS programs demonstrated polymer-based systems having 100000 to 1 million

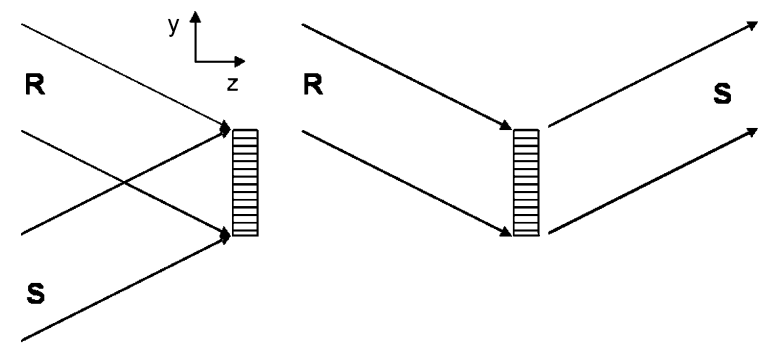

(a)

(b)

Fig. 4. Holographic optics processes for: (a) recording and (b) readout.

times better data storage capacity and transfer rates than the first digital HDSS developed by Stanford in 1994 [24], using $\mathrm{LiNbO}_{3}$ as the recording medium.

Finally, in Section I, we have reviewed the motivation, potential, and research activity on HDSSs. In Section II, we review the fundamentals of holographic optics, including reciprocal space, also called $k$-space, formalism. We compare several basic multiplexing architectures to illustrate key properties of holographic optics and conclude with several more advance architectures that have been implemented in various demonstrations during the last decade. In Section III, we review one of the unique features of HDSS, namely, an extremely fast associative search capability. In Section IV, we review the basic material tradeoffs for an HDSS, including the tradeoffs among sensitivity, robustness during readout, and permanency. In Section V, we review specific details of photorefractive materials, typically crystals, including techniques for nondestructive readout. In Section VI, we review specific details of photopolymer materials, including sensitivity, shrinkage, and media stability. In Section VII, we review various optical system architectures with a view toward capacity, transfer rates, and access times. In Section VIII, we review various signal processing approaches, including data modulation and error correction. In Section IX, we review critical issues in the electronics subsystem required to support an HDSS. In Section X, we review various demonstrations completed in the last decade, including both page based and single bit holographic data storage. In Section XI, we comment on the outlook of future opportunities in this field. In Section XII, we summarize the overview presented here.

\section{HOLOGRAPHIC OPTICS}

\section{A. Fundamental Concepts}

1) Hologram Recording: Holograms are recorded using a reference beam and a signal beam comprising data. As shown in Fig. 4(a), the reference beam $R$ and signal beam $S$ interfere to generate a fringe pattern, which is transferred to the medium. In HDSSs, the recorded hologram typically takes the form of an index or phase grating. As shown in Fig. 4(b), readout is accomplished by illuminating the hologram with the original reference beam $R$, which diffracts of the recorded hologram to generate an output signal beam 


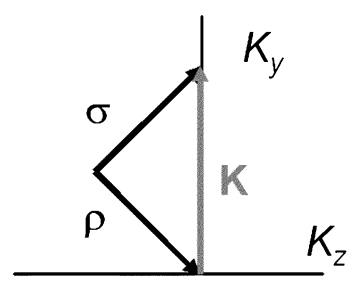

Fig. 5. Relationship between wave vectors of signal and reference beam and grating vector.

$S$. The electric field amplitude of the propagating reference wave is represented as

$$
R=R_{0} \exp (i \boldsymbol{\rho} \cdot \mathbf{r}) .
$$

$R_{0}$ is the complex amplitude, $\boldsymbol{\rho}$ is the wave vector, and $\mathbf{r}$ is the spatial dimension. The electric field amplitude of the propagating signal wave is similarly represented as

$$
S=S_{0} \exp (i \boldsymbol{\sigma} \cdot \mathbf{r})
$$

where $S_{0}$ is the complex amplitude and $\boldsymbol{\sigma}$ is the wave vector. The interfering signal and reference wave vectors generate a grating with a grating wave vector $\mathbf{K}$ given by

$$
\mathbf{K}=\boldsymbol{\sigma}-\boldsymbol{\rho} .
$$

This relationship is illustrated in Fig. 5. The resulting hologram is given by the following index grating:

$$
n(y)=n_{0}+\operatorname{Re}[\delta n \exp (i K y)]
$$

in which $y$ is the transverse direction of propagation and $\delta n$ is a complex coefficient given by

$$
\delta n=\frac{n_{1} R_{0} S_{0}^{*}}{I_{0}}
$$

where $n_{1}$ is the proportionality constant that accounts for the material response to illumination and $I_{0}$ is the total illumination.

2) Diffraction Efficiency: The diffraction efficiency of a recorded hologram is given by

$$
\eta=\sin ^{2}\left(\hat{\mathbf{e}}_{R} \cdot \hat{\mathbf{e}}_{S} \frac{\pi|\delta n| L}{\lambda \cos \theta}\right) \exp (-\alpha L)
$$

where $L$ is the thickness of the medium, $\alpha$ is the absorption coefficient of the material, and $\hat{\mathbf{e}}_{R}$ and $\hat{\mathbf{e}}_{S}$ are the polarizations of the reference and signal beams, respectively.

Note that the diffraction efficiency can approach $100 \%$ for relatively strong holograms. For relative weak holograms, with negligible absorption, the diffraction efficiency can be represented as

$$
\eta=\left(\hat{\mathbf{e}}_{R} \cdot \hat{\mathbf{e}}_{S} \frac{\pi|\delta n| L}{\lambda \cos \theta}\right)^{2} .
$$

3) Bragg Selectivity: Holograms recorded in a material having a substantial thickness display a property called Bragg selectivity. Bragg selectivity means that holograms recorded with a particular, for example planar, reference

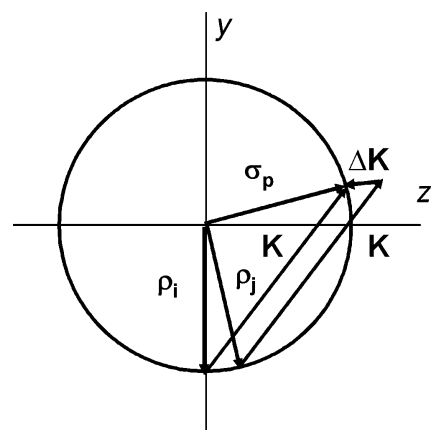

Fig. 6. Bragg mismatch illustrated upon rotation of the reference beam wave vector.

wave vector provided the strongest diffraction efficiency when read out with the same reference beam. The original reference beam and the hologram are said to be Bragg matched. When the wave vector of the readout reference beam is substantially different, the diffraction efficiency is significantly less. The wave vector of the reference beam can be changed by changing the angle of incidence or wavelength of the reference beam, or both. Bragg selectivity, therefore, allows multiple holograms to be recorded in an HDSS in the same volume of media, because only the hologram Bragg matched to the readout beam is reconstructed with substantial diffraction efficiency; all of the others are substantially attenuated.

Bragg selectivity is illustrated in Fig. 6. Signal beam wave vector $\boldsymbol{\sigma}$ and reference beam wave vector $\boldsymbol{\rho}_{i}$ record a hologram grating having wave vector $\mathbf{K}$. In an isotropic medium, wave vectors $\boldsymbol{\sigma}$ and $\boldsymbol{\rho}_{i}$ have the same wavenumber $\beta=2 n \pi / \lambda$ and, therefore, reside on the Ewald sphere. Because the original signal and reference wave vector are Bragg matched, the vector sum lies on the Ewald sphere

$$
\sigma=\mathbf{K}+\boldsymbol{\rho} .
$$

A second reference beam with wave vector $\rho_{j}$ diffracts off of a grating with wave vector $\mathbf{K}$; the resulting vector sum differs from the output signal by $\Delta \mathbf{K}$

$$
\sigma=\mathbf{K}+\boldsymbol{\rho}+\Delta \mathbf{K} .
$$

The second reference beam is, therefore, not Bragg matched to the grating and is, therefore, substantially attenuated. When the wavelength is the same and the angle of the reference beam changes, as is the case for angle multiplexing, the Bragg selectivity is approximated as

$$
\eta=\left(\hat{\mathbf{e}}_{R} \cdot \hat{\mathbf{e}}_{S} \frac{\pi|\delta n| L}{\lambda \cos \theta}\right)^{2} \operatorname{sinc}^{2}\left(\frac{\Delta K_{z} L}{2}\right)
$$

where $\Delta K_{z}$ is projection of $\Delta \mathbf{K}$ on the $z$ axis and $\operatorname{sinc}(x)=$ $\sin (\pi x) / \pi x$. Thus, Bragg selectivity may be characterized by a minimum separation of grating vectors along the $z$ axis, given by $\left(\Delta K_{z}\right)_{\min }=2 \pi / L$. The first null of the sinc function is obtained at this separation vector. The finite lateral extent of the medium puts a similar constraint on the minimum separation of grating vectors in the $x$ and $y$ axis, so that the corresponding minimum separation vectors are given by 


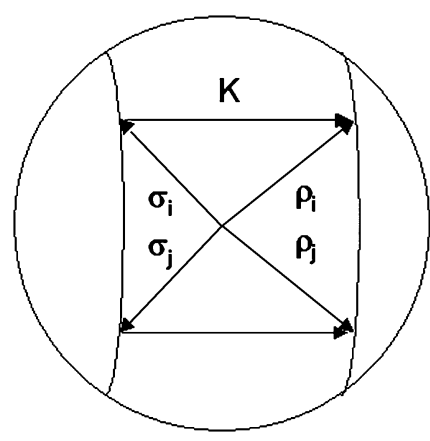

Fig. 7. Bragg degeneracy arrangement.

$\left(\Delta K_{x}\right)_{\min }=2 \pi / H$ and $\left(\Delta K_{y}\right)_{\min }=2 \pi / W$, respectively, for medium height $H$ and width $W$, respectively.

When multiple hologram gratings are recorded to increase the capacity of a storage medium, the Bragg selectivity results in significant crosstalk between the holograms. As will be shown in subsequent sections, holographic data storage architectures are generally designed to minimize this crosstalk due to the finite size of the medium.

The crosstalk between gratings is generally geometry dependent, and we need to consider the concept of degenerate gratings, frequently called Bragg degeneracies. Fig. 7 illustrates how two different pairs of signal and reference beams $\left(\boldsymbol{\sigma}_{i}, \boldsymbol{\rho}_{i}\right)$ and $\left(\boldsymbol{\sigma}_{j}, \boldsymbol{\rho}_{j}\right)$ can write gratings having exactly the same wave vector. In this case, any information stored using this wave vector is superimposed, leading to prohibitively high crosstalk. The solution to this problem is to carefully design an architecture to manage grating overlap. Straightforward examples include angular multiplexing based on a set of reference beams having coplanar wave vectors and wavelength multiplexing with a reference beam oriented in a fixed direction. These will be discussed in detail in subsequent sections. More sophisticated techniques have been proposed for reference beam sets as well as coded, nonplanar reference beams [25]-[27].

4) Dynamic Range: When multiple holograms are recorded in a material, the dynamic range of the index grating is divided up among the holograms. This dynamic range is characterized by an $M \#$, which is defined as

$$
M \#=\sum_{i=1}^{M} \eta_{i}^{\frac{1}{2}}
$$

in which $N$ is the total number of holograms and $\eta_{i}$ is the diffraction efficiency of the $i$ th hologram. In an ideal material, the $M \#$ is independent of $N$ for a large number of multiplexed holograms. In this case, the $M \#$ is given by

$$
M \#=\hat{\mathbf{e}}_{R} \cdot \hat{\mathbf{e}}_{S} \frac{\pi|\delta n| L}{\lambda \cos \theta} .
$$

The diffraction of each hologram is a system of $N$ multiplexed holograms is, therefore, given by

$$
\eta=\left(\frac{M \#}{N}\right)^{2}
$$

(a)

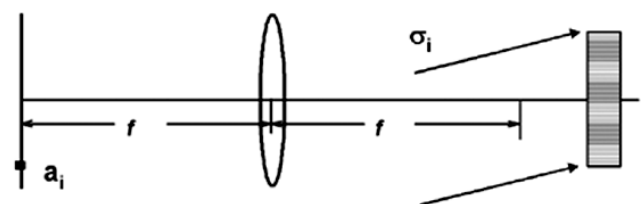

(b)

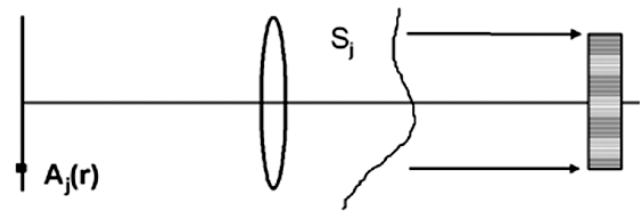

Fig. 8. Fourier transform configuration for holographic data storage. (a) Point source. (b) SLM.

5) Material and Sample Sensitivity: Another important property of materials used in HDSSs is the recording sensitivity. The material sensitivity, typically measured near $t^{\sim} 0$, can be determined using

$$
S=\frac{\frac{\partial(\sqrt{\eta})}{\partial t}}{I_{0} L} .
$$

The material sensitivity depends on material parameters and recording configuration and is generally reported in centimeters per joule. The sample sensitivity can be determined using

$$
S^{\prime}=\frac{\frac{\partial(\sqrt{\eta})}{\partial t}}{I_{0} t}
$$

where $t$ is the exposure time. The sample sensitivity depends on the material thickness and is generally reported in square centimeters per joule. (The type of sensitivity referenced can be generally inferred from the units reported.)

Inorganic photorefractive materials such as lithium niobate have sample sensitivity ranging from 0.01 to $0.1 \mathrm{~cm}^{2} / \mathrm{J}$ and $M \#$ of about 1-10 for 1-cm-thick samples. Polaroid ULSH-500 photopolymers have sample sensitivity ranging from $20 \mathrm{~cm}^{2} / \mathrm{J}$ and $M \#$ as high as 10 for 0.5 -mm-thick samples. Development of stronger, more sensitive, and more stable holographic storage materials is an active area of research.

\section{B. Fourier Transform Configuration}

A particularly advantageous configuration for holographic data storage utilizes the Fourier transform properties of a spherical lens. In the Fourier transform configuration, shown in Fig. 8, the signal path comprises a signal page generated by an SLM situated at the front focal plane of a lens, situated a focal length $f$ in front of the lens. The holographic recording material is situated near the rear focal plane of the lens, but not necessarily at the focal plane. Fig. 8(a) shows that point source $a_{i}$ becomes a plane wave on passing through the lens. More generally, Fig. 8(b) shows an SLM having a spatial distribution given by $A_{j}(\mathbf{r})$, for position vector $\mathbf{r}$, which is transformed into a spectrum of wave vectors according to the relationship

$$
S_{j}(\mathbf{r})=\int d \boldsymbol{\sigma} \exp (i \boldsymbol{\sigma} \cdot \mathbf{r}) A_{j}\left(\frac{2 \pi f \boldsymbol{\sigma}}{\lambda}\right) .
$$




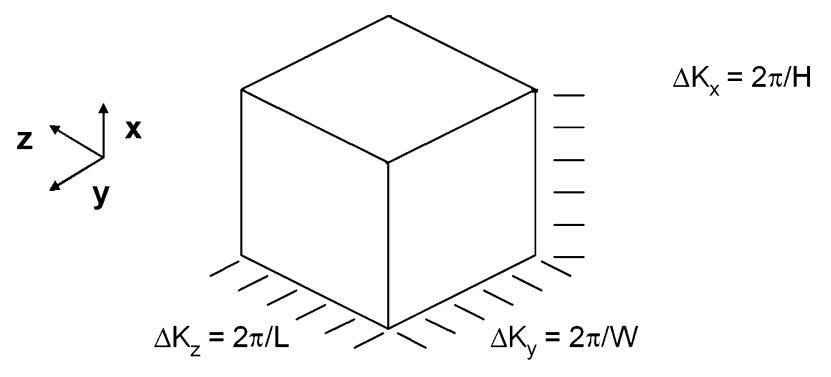

Fig. 9. Grating wave vector resolution in $k$-space.

Thus, in this configuration, each pixel corresponds to a plane wave in the holographic storage material. A recorded image of the signal page is imaged onto a detector array using an appropriate lens. In the Fourier transform configuration, the signal page is distributed throughout the holographic storage material and is, therefore, robust against local defects in the material. This configuration is relatively insensitive to displacements of the holographic storage medium, provided it remains in the same orientation.

\section{Grating Vectors as Data Channels}

The data capacity can be estimated using a degrees-of-freedom model based on resolvable grating vectors [26], [28]. The finite extent of a holographic storage medium generally limits the resolution of grating vectors. Fig. 9 illustrated the resolution of grating vectors diagrammatically. In the direction of signal propagation, the grating vector resolution is given by $\Delta K_{z}=2 \pi / L$; in the directions perpendicular to the direction of signal propagation, grating vectors are given by $\Delta K_{y}=2 \pi / W$ and $\Delta K_{x}=2 \pi / H$. Here, $L, W$, and $H$ correspond to the medium length, width, and height, respectively.

In an isotropic medium, the maximum wave vector length is given by $K=2 \pi n / \lambda$, which defines a radius of a grating vector sphere that bounds the space of allowable grating vectors. The volume of this space is given by

$$
V_{K}=\frac{4 \pi}{3} K^{3}
$$

Defining a data channel as a resolvable grating vector, the total number of accessible data channels is given by $Q=$ $V_{k} /\left(\Delta K_{x} \Delta K_{y} \Delta K_{z}\right)$ so that

$$
Q=\frac{32 \pi}{3} \frac{n^{3} V}{\lambda^{3}}
$$

where $\mathrm{V}$ is the volume of the holographic recording medium. This quantity allows two data channels in quadrature for each grating orientation, which generally requires coherent demodulation to separate the data channels. For one data channel in each grating direction, the total number of accessible data channels is given by [26] and [28]

$$
Q=\frac{16 \pi}{3} \frac{n^{3} V}{\lambda^{3}}
$$

Equation (19) indicates that a material with an index of refraction of 2.5, such as lithium niobate, has a channel density of $2 \times 10^{15}$ resolvable grating vectors. In a practical application, the number of degrees of freedom is reduced by a factor $\alpha$ to $10^{12}$ to $10^{13}$ due the limited NA of a signal beam and limited angular excursion of the reference beam. In an HDSS, the accessible data channels $\alpha Q$ are the product of the number of pages $N$ and the number of pixels per page $P$. We assume here a code rate of one, or, in other words, that there is no extra overhead associated with adding error correction and channel coding bits. In practice this is not true, and the code rate is smaller than one, typically around 0.75 . Once we have determined the number of data channels, we may then estimate total capacity through the Shannon law, namely, by multiplying the total number of channels by the capacity of a single channel

$$
C_{\text {total }}=\alpha Q \log _{2}(1+\mathrm{SNR})=N P \log _{2}(1+\mathrm{SNR})
$$

in which $N$ is the number of pages and $P$ is the number of pixels per page, and SNR denotes signal-to-noise ratio. For a white Gaussian noise model, given the constraint of constant laser power, and for constant total diffraction efficiency, the SNR decreases with increasing number of pixels per page $(\mathrm{SNR} \sim 1 / P)$ At the same time, due to a limited dynamic range, the total diffraction efficiency decreases as $1 / N^{2}$, leading to a corresponding behavior of the SNR $\left(\mathrm{SNR} \sim 1 / N^{2}\right)$. Thus, there exists a tradeoff between the SNR and the number of pages which can be superimposed. As a result, given parameters of the system (laser power, optical performance, electronic detection noise, media $M \#$, and data rate), there is an optimum number of pages which need to be written in a single location of the volume holographic memory for which the capacity $C_{\text {total }}$ is maximized [29] (Fig. 10). For realistic parameters of storage in the $90^{\circ}$ geometry lithium niobate system $\left(P=10^{6}, M \#=1\right.$, laser power $1 \mathrm{~W}$ ) the theoretical upper bound on the optimum number of pages is estimated to be $\sim 2500$, with total raw capacity per storage location of $\sim 2.5 \times 10^{9} \mathrm{~b}$ [9].

It is worth noting that the capacity calculated above is merely an information theory upper bound and does not consider the effects of SNR reduction due to nonideal imaging and interpixel and interpage crosstalk. The state-of-the-art demonstration of digital hologram recording in the $90^{\circ}$ geometry in lithium niobate [30] experimentally realized a capacity of $10^{9}$ pixels per storage location (i.e., 1000 superimposed holograms of 1-megapixel images). The resulting raw channel density of $\sim 350 \mathrm{~b} / \mu \mathrm{m}^{2}$ (calculated as the ratio of capacity and the area occupied by the holograms on the entrance face of the storage crystal) represented, however, only $\sim 1.08 \%$ of the theoretical volumetric density limit of $1 / \lambda^{3}$.

\section{Multiplexing Techniques}

1) Angular Multiplexing: Angular multiplexing consists of hologram formation in which each reference wave consists of a plane wave of the same wavelength incident at a different angle. A reference beam selected from the set of 


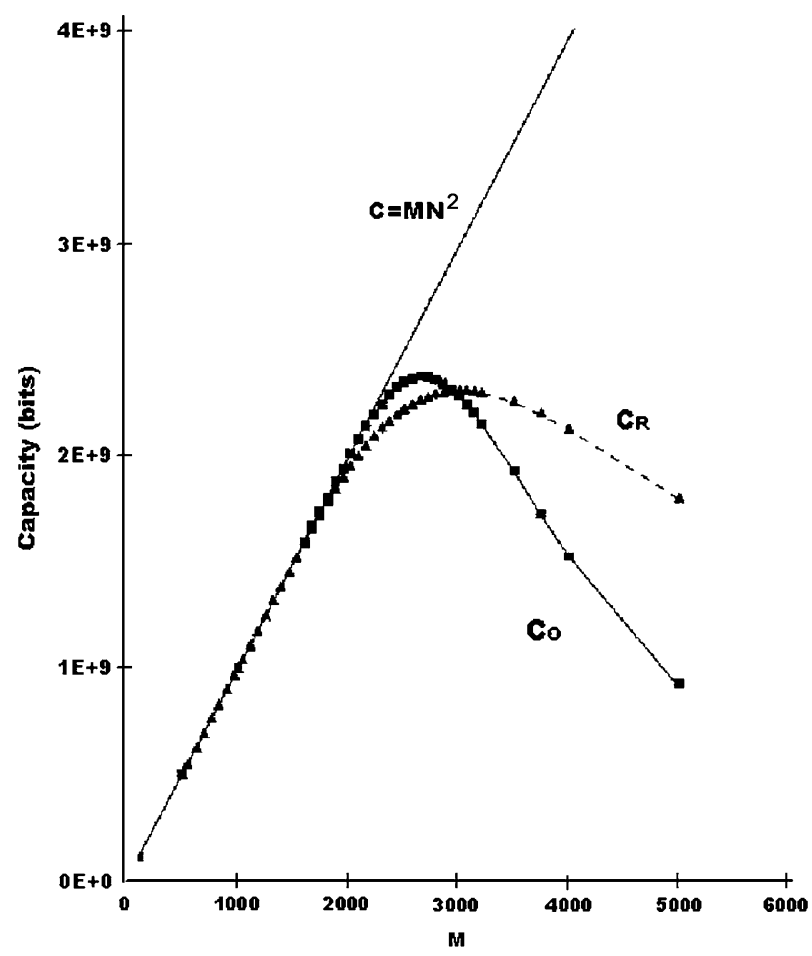

Fig. 10. Capacity versus number of superimposed data pages (courtesy of M. Neifeld, University of Arizona [29]). $N$ denotes in this case the number of pixels per page, and $M$ represents the number of superimposed holograms.

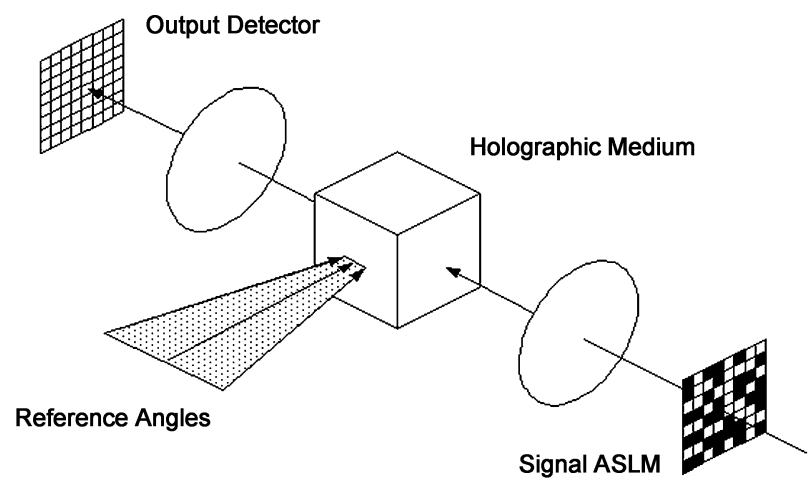

Fig. 11. General angle multiplexing architecture.

reference waves used to multiplex holograms at different angles is given by

$$
R_{l}=\frac{A_{l}}{2} \exp \left[i\left(\boldsymbol{\rho}_{l} \cdot \mathbf{r}-\omega t\right)\right]+\frac{A_{l}^{*}}{2} \exp \left[-i\left(\boldsymbol{\rho}_{l} \cdot \mathbf{r}-\omega t\right)\right]
$$

in which $l$ is the index of the reference beam, $\rho_{l}$ is the wave vector, $\left|\boldsymbol{\rho}_{l}\right|$ does not depend on $l, \omega$ is the angular frequency, $t$ is time, and $A_{l}$ is the amplitude of the plane wave.

Fig. 11 shows a diagram for angular multiplexing. Data are encoded on a signal beam using a signal amplitude SLM (signal ALSM). The appropriate data pattern is loaded onto the signal ALSM through an appropriate electronic interface to a computer or peripheral system. The signal ALSM is situated at the front focal plane of a lens; the modulated signal propagates to the holographic medium in a Fourier transform configuration. Reference beams are plane waves incident on the holographic medium. Each reference vector is limited to a plane of incidence further situated so that the reference beams and an included wave vector in the signal beam lie in the same plane, as shown in Fig. 11. A separate hologram is recorded with each reference wave, resulting in multiplexed holographic data pages. A data page is read out by illuminating the holographic medium with one of the reference beams. The output data page is focused on the output detector, and then demodulated by the appropriate electronics.

Throughout this paper, we are primarily interested in pageoriented multiplexing, in which we organize a large quantity of data recorded as pixels of an image. Each pixel stored by each reference beam may be considered to be a separate data channel. Angular selectivity is maximized for signal and reference wave vectors that are normally incident to perpendicular faces of the holographic storage medium [8], [31]-[33]. For copolarized signal and reference beams, the diffraction efficiency is expressed as

$$
\eta=\left(\frac{\pi|\delta n| L}{\lambda \cos \theta}\right)^{2} \operatorname{sinc}^{2}\left(\frac{\pi L \sin \left(\theta_{\boldsymbol{\rho}}-\theta_{\boldsymbol{\sigma}}\right)}{\lambda \cos \theta_{\boldsymbol{\sigma}}} \Delta \theta\right)
$$

where $\theta_{\boldsymbol{\rho}}$ is the mean angle of the first and second reference beam and $\theta_{\boldsymbol{\sigma}}$ of incidence of the signal beam. The minimum separation angle is defined as the first extinction of the sinc function, which occurs at

$$
\Delta \theta=\frac{\lambda \cos \theta_{\boldsymbol{\sigma}}}{n L \sin \left(\theta_{\boldsymbol{\rho}}-\theta_{\boldsymbol{\sigma}}\right)} .
$$

In this expression, the separation angle refers to the angle inside the material; refraction at the surface of materials having a high index of refraction will substantially increase the minimum separation angle.

For transmission holograms, in which the signal and reference beam are incident at the same side of the material, the angle between the signal and reference beams is relatively small, especially in media displaying a high index of refraction, and angular selectivity is moderate. Angular selectivity is highest for the perpendicular geometry, in which the signal and reference beam are incident at perpendicular surfaces of a holographic storage medium. The dependence of separation angle as a function of the angle between the signal and reference beams is shown in Fig. 12. In the perpendicular geometry, the minimum separation angle outside the holographic material is given by

$$
\Delta \theta=\frac{\lambda}{L}
$$

In a holographic storage material such as lithium niobate having a thickness of $1 \mathrm{~cm}$, the angular selectivity is $50 \mu \mathrm{rad}$ in free space for recording/readout wavelengths of $500 \mathrm{~nm}$, in the perpendicular geometry. Thus, 10000 holograms can be accessed with an angular range of the reference beam of about $30^{\circ}$.

For moderate, nonoverlapping angular spectra of the signal and reference beams, we can derive a simple expression to estimate the accessible data channels. A reference wave illuminating the side face of the crystal, therefore, has a maximum number of degrees of freedom, or number of 

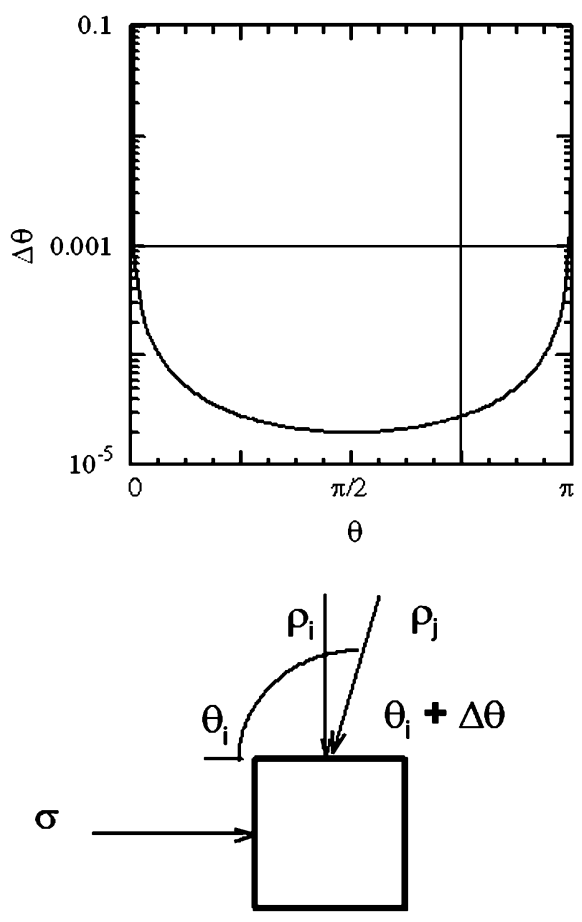

Fig. 12. Angular selectivity as illustrated by separation angle as a function of angle between the signal and reference beam, in a holographic storage material such as lithium niobate having a thickness of $1 \mathrm{~cm}$, an index of refraction of 2.5 , and recording/readout wavelengths of $500 \mathrm{~nm}$.

grating vectors separated by the minimum separation vectors, of $Q_{z}=2 n L / \lambda$. A signal wave illuminating a face has a maximum number of degrees of freedom $Q_{x}=2 n H / \lambda$ in the $x$ dimension and $Q_{y}=2 n W / \lambda$ in the $y$ dimension. In this geometry, the number of accessible data channels is further estimated by taking into account the angular bandwidths of the reference and signal beam to yield

$$
Q=\frac{8 V}{\lambda^{3}}(\mathrm{NA})^{2} \sin \frac{\Theta}{2}
$$

where NA is the numerical aperture of a signal incident at the surface normal and $\Theta$ is the angular range of the reference beam, both in free space outside the material. For a wavelength of $500 \mathrm{~nm}$, a signal NA of 0.25 , and reference beam angular range of $30^{\circ}$, the number of data channels is about $10^{12} \mathrm{~cm}^{-3}$.

Crosstalk: A practical HDSS generally displays deviation from the grating vector separation rules identified above. The result is crosstalk between multiplexed holograms. To understand the impact of crosstalk, we use a reciprocal space, or $k$-space, formalism [9], [28], [32], [34]-[36]. A signal beam comprising a data page and a reference beam generate a hologram having spectrum of grating wave vectors. This spectrum is shown in the shaded arc in Fig. 13, which depicts a cross section of $k$-space. In general, the spectrum of grating vectors comprises a solid cross section of a sphere. Multiple holograms are recorded by varying the incident angle of the reference beam so that the wave vector rotates. The result is a series of grating vector spectra indicated in Fig. 14. An important observation in Fig. 14 is that the grating vector sep-

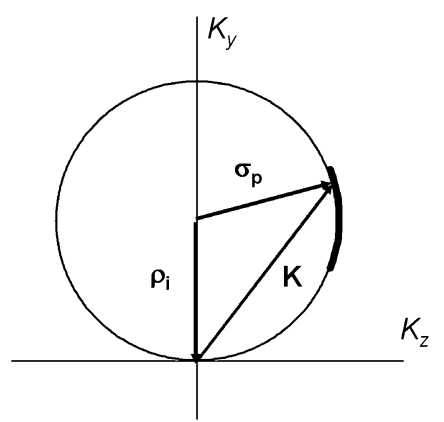

Fig. 13. Ewald sphere showing relationship between signal wave vector $\sigma_{p}$, reference wave vector $\rho_{i}$, and grating wave vector $K$.

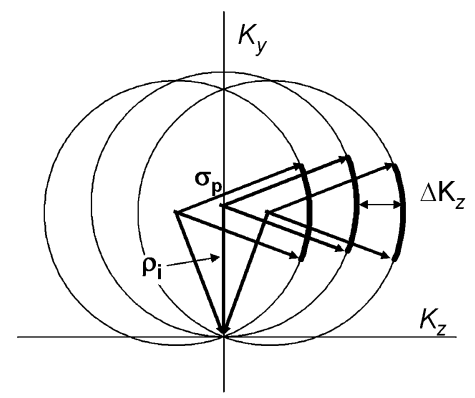

Fig. 14. $k$-space representation of angle multiplexing.

aration $\Delta K_{z}$ between the spectra of two holograms is not constant at various positions in the spectra, so that the optimum grating vector separation of a multiple of $\left(\Delta K_{z}\right)_{\min }$ is not the same for all grating vectors in the spectrum. Thus, the null of the Bragg selectivity sinc function cannot be met over the entire signal spectrum, resulting in crosstalk.

This origin of crosstalk is illustrated in a different way in Fig. 15. A principal signal wave vector $\sigma_{p}$ and a principal reference wave vector $\rho_{i}$ are incident in the perpendicular geometry. For the principal wave vector, an angular separation of $\Delta \theta$ is selected to correspond to the minimum separation grating vector projection on the $z$ axis $\Delta K_{z}$. Thus, the peak readout angle of each hologram is situated at the null angles of each of the other multiplexed holograms. For another signal wave vector $\sigma_{q}$, however, the peak readout angle is not situated at the null angles of each of the other multiplexed holograms, resulting in crosstalk.

Crosstalk is minimized when the signal and reference beams are incident on perpendicular surfaces of the medium, for which the ratio between the crosstalk and signal, the crosstalk limited SNR, is given by [31]

$$
\mathrm{SNR} \sim \frac{Q_{z}}{N(\mathrm{NA})}
$$

in which $N$ is the number of holograms and NA is the numerical aperture of the signal beam. When discrete pixels are stored in a Fourier transform arrangement, so that rows of pixels span the dimension parallel to the reference wave vectors, the relative crosstalk takes on the form [32]

$$
\mathrm{SNR} \sim \frac{Q_{z} Q_{y}}{N P_{y}}
$$


(a)

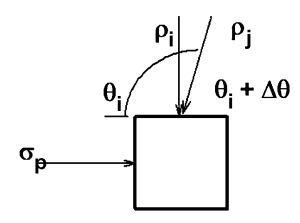

Other Signal Wavevector

(b)

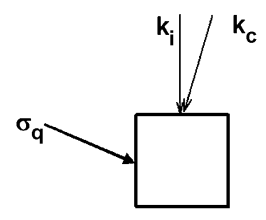

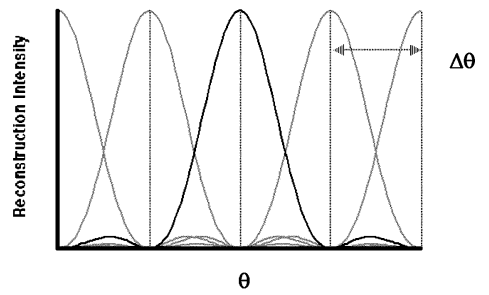

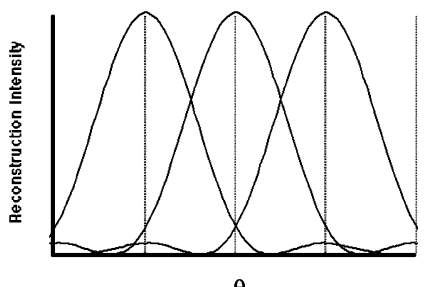

Fig. 15. Angle multiplexing properties for: (a) normally incident signal wave vector and (b) oblique signal wave vector.

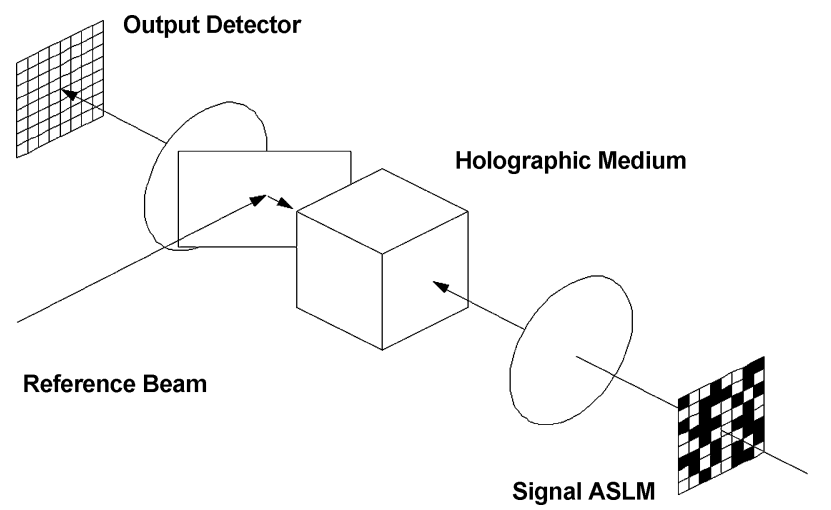

Fig. 16. Generic wavelength multiplexing architecture.

in which $P_{y}$ is the number of columns of pixels.

2) Wavelength Multiplexing: Wavelength multiplexing consists of hologram formation in which each reference wave consists of a plane wave of a different wavelength incident at the same angle, such that

$$
R_{l}=\frac{A_{l}}{2} \exp \left[i\left(\boldsymbol{\rho}_{l} \cdot \mathbf{r}-\omega_{l} t\right)\right]+\frac{A_{l}^{*}}{2} \exp \left[-i\left(\boldsymbol{\rho}_{l} \cdot \mathbf{r}-\omega_{l} t\right)\right]
$$

where now $\left|\boldsymbol{\rho}_{l}\right|$ and the corresponding angular frequency $\omega_{l}$ vary but the orientation of $\rho_{l}$ is the same for all reference beams. Only the recording reference waves are Bragg 1 matched, and any others result in Bragg-mismatched reconstruction.

Fig. 16 shows a diagram for wavelength multiplexing. As with the angular multiplexing case, a Fourier transform configuration is used. The signal and reference beams are incident on opposite sides of the holographic medium, known as a reflection configuration. Wavelength selectivity is maximized for included signal and reference wave vectors corresponding to counterpropagating directions in the holographic storage medium [8], [31]-[33].

Fig. 17 shows the geometry in which the reference beam is incident normal to the surface of a medium and the signal beam is incident at an angle $\theta$ with respect to the reference (a)

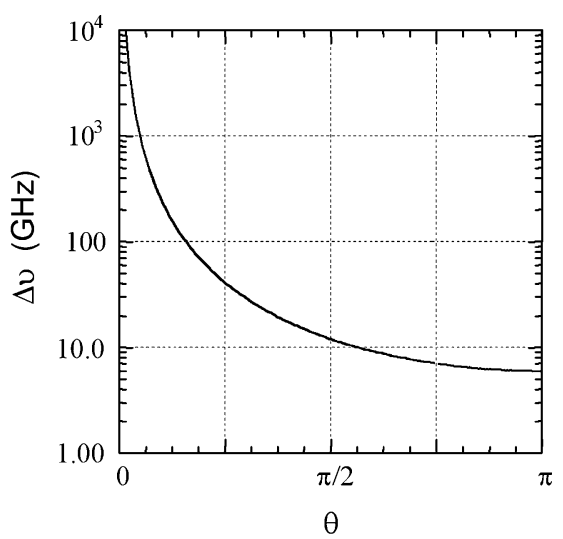

(b)

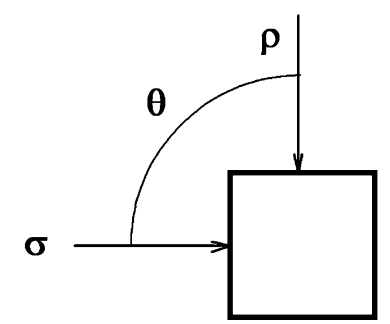

Fig. 17. Frequency selectivity as illustrated by separation angle as a function of angle between the signal and reference beam, in a holographic medium such as $\mathrm{LiNbO}_{3}$ having a thickness of $1 \mathrm{~cm}$, an index of refraction of 2.5 , and recording and readout wavelengths of $500 \mathrm{~nm}$.

beam. For copolarized signal and reference beams, the diffraction efficiency is expressed as

$$
\eta=\left(\frac{\pi|\delta n| L}{\lambda \cos \theta}\right)^{2} \operatorname{sinc}^{2}\left(\frac{2 \pi n L \sin ^{2}\left(\frac{\theta}{2}\right)}{c} \Delta \nu\right)
$$

where $\Delta \nu$ is frequency difference between the recording and readout reference beam, $c$ is the velocity of light in free space, and $\theta$ is the angle between a planar signal and reference wave in the medium. For reflection holograms, the minimum separation frequency is defined as the first extinction of the sinc function, which occurs at

$$
\Delta \nu=\frac{c}{2 n L \sin ^{2}\left(\frac{\theta}{2}\right)} .
$$




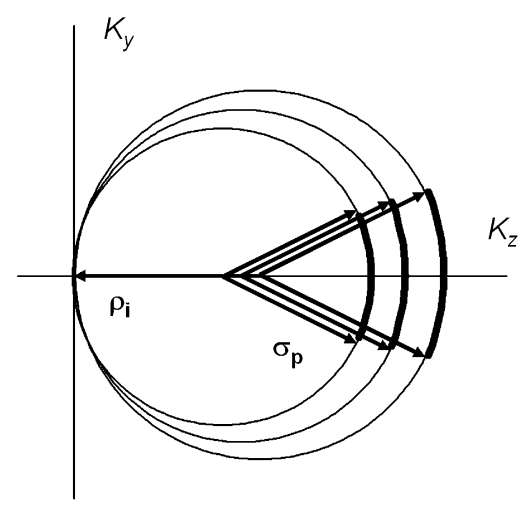

Fig. 18. $k$-space representation of wavelength multiplexing.

For reflection holograms, the angle between the signal and reference beam is generally substantial. Frequency selectivity is highest for the counterpropagating geometry, in which the signal and reference beam are incident at opposite surfaces of a holographic storage medium and propagate in opposite directions. The dependence of separation frequency as a function of the angle between the signal and reference beams is shown in Fig. 17. For counterpropagating signal and reference beams, the minimum separation frequency is

$$
\Delta \nu=\frac{c}{2 n L} \text {. }
$$

In a holographic storage material such as lithium niobate having a thickness of $1 \mathrm{~cm}$ and an index of refraction of 2.5 , the frequency selectivity is $6 \mathrm{GHz}$ for recording/readout wavelengths of $500 \mathrm{~nm}$. Thus, over 10000 holograms can be accessed with a wavelength range of the reference beam from 500 to $560 \mathrm{~nm}$.

Wavelength multiplexing generally displays crosstalk between multiplexed holograms due to deviations from the wavelength selectivity rules identified above. The series of grating vector spectra for wavelength multiplexed holograms is indicated by bold line arcs in Fig. 18. As with angular multiplexing, the grating vector separation $\Delta K_{z}$ between the spectra of two holograms is not constant at various positions in the spectra, so that the optimum grating vector separation of a multiple of $\left(\Delta K_{z}\right)_{\min }$ is not the same for all grating vectors in the spectrum. Thus, the null of the Bragg selectivity sinc function cannot be met over the entire signal spectrum, resulting in crosstalk.

Crosstalk is minimized when the signal and reference beams are counterpropagating, for which the ratio between the crosstalk and signal, crosstalk limited SNR, is given by [37]

$$
\mathrm{SNR} \sim \frac{4}{(\mathrm{NA})^{2}}
$$

in which NA is the numerical aperture of the signal beam. Note that for this configuration, the crosstalk limited SNR does not depend on the number of multiplexed holograms, owing to the arrangement in reciprocal space.

In practical systems, crosstalk is not the limiting factor. Rather, wavelength multiplexing is limited by stable tunable laser sources and strong backscatter in holographic media.

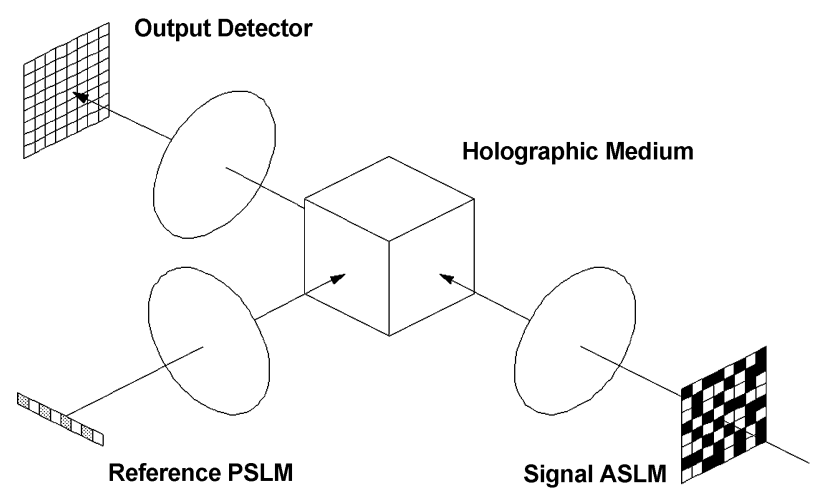

Fig. 19. Generic HDSS using phase-encoded multiplexing.

One suggested approach to address the issue of backscatter is to use a perpendicular geometry, which has wavelength selectivity comparable to the counterpropagating geometry, as shown in Fig. 17 [38]; for moderate capacities, crosstalk is not a factor.

3) Phase-Encoded Multiplexing: Phase-encoded multiplexing uses a phase SLM to control the reference beam and requires accurate control of the phase modulation and efficient collection of phase patterns to minimize crosstalk. An example architecture for phase-encoded multiplexing is shown in Fig. 19. Here, a Fourier transform arrangement is used for both the signal and reference beam paths. A phase-encoded reference beam comprises a phase pattern $e^{\phi(x, y)}$ established by an SLM. In this example, a one-dimensional (1-D) SLM is situated at the front focal plane of the lens, so that the resulting reference beam incident on the material has a wave vector spectrum in one dimension. By using a set of orthonormal functions and an appropriate optical configuration, multiple holograms may be superimposed without significant crosstalk.

Multiple holograms are recorded by varying the phase codes of the reference beam. For each hologram, the result is the grating vector spectrum indicated by the shaded region in Fig. 20. In contrast to angular multiplexing, the grating vector spectra for each hologram overlap; what distinguished each hologram are the phase relationships among the recorded holograms. Krile et al. [39], [40] and Morozov [41] have independently outlined this procedure for thin holograms, for which there is no Bragg selectivity. Because of its simplicity, we examine this procedure to illustrate basic properties of phase encoding. When volume effects are taken into account, Bragg selectivity reduces crosstalk.

In the general case, a two-dimensional (2-D) phase pattern may be used. The transmittance of the medium with a linear response is given by

$$
t=\mathcal{A} \sum_{l=1}^{N}\left|R_{l}+S_{l}\right|^{2}
$$

where $S_{l}\left(k_{x}, k_{y}\right)$ and $R_{l}\left(k_{x}, k_{y}\right)$ denote the Fourier transforms of the signal $s_{j}(x, y)$ and the coded reference $r_{j}(x, y)$, respectively, at the plane of the SLM, and $\mathcal{A}$ is a proportionality constant dependent on the strength of the hologram. 


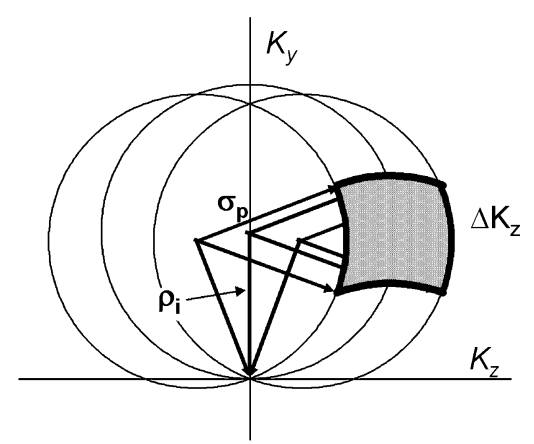

Fig. 20. $k$-space representation of phase-encoded multiplexing.

Upon illumination with reference beam $r_{c}$, light diffracted from the hologram is given by

$$
D=t R_{c}
$$

where $R_{c}\left(k_{x}, k_{y}\right)$ is the readout reference beam. If a reference and signal pair $r_{l}$ and $s_{l}$ are used for recording the $l$ th hologram, then upon readout with $r_{c}$ we arrive at

$$
\begin{aligned}
D= & t R_{c} \\
= & a \sum_{l=1}^{N} S_{l} R_{l}^{*} R_{c}+a \sum_{l=1}^{N} S_{l}^{*} R_{l} R_{c} \\
& +a \sum_{l=1}^{N}\left[\left|R_{l}\right|^{2}+\left|S_{l}\right|^{2}\right] R_{c} .
\end{aligned}
$$

The first summation gives the reconstructed wave of interest. If one hologram is stored, the diffracted beam that passes through the Fourier transforming lens to produce the field is [42]

$$
d=a s_{1} *\left(r_{1} \star r_{c}\right)
$$

at the detector plane, where the operators $\star$ and $*$ denote correlation and convolution, respectively, and $a$ is a proportionality constant. A sharp autocorrelation peak is required in order to reconstruct the signal $s_{1}$. For many object pairs this expression is generalized to

$$
d_{c}=a \sum_{l=1}^{N} s_{l} *\left(r_{l} \star r_{c}\right) .
$$

If the cross correlation between two reference codes $r_{i}$ and $r_{j}$ is appreciable, then we have significant crosstalk between two images stored with these codes [42]. Krile et al. have shown that this objective can be efficiently accomplished using Gold and Carter codes developed for spread spectrum communication applications [40].

Kral et al. [43] have shown that the lower limit for the crosstalk-limited SNR for holograms multiplexed in thin media is given by

$$
\mathrm{SNR}^{\sim} \frac{1}{N}
$$

The SNR can be improved using techniques outlined by Kral et al., including illumination of a phase pattern with a spherical reference beam.
For the case illustrated in Fig. 19, the reference wave would ideally be composed of point sources corresponding to a pixel in the plane of the phase SLM. In the Fourier transform geometry illustrated here, each point source corresponds to discrete constituent plane waves after the Fourier transform lens, such that

$$
\begin{aligned}
R_{l}(\mathbf{r})=\sum_{j}\left\{\frac{R_{l j}}{2}\right. & \exp \left[i\left(\boldsymbol{\rho}_{j} \cdot \mathbf{r}-\omega t\right)\right] \\
& \left.+\frac{R_{l j}^{*}}{2} \exp \left[-i\left(\boldsymbol{\rho}_{j} \cdot \mathbf{r}-\omega t\right)\right]\right\} .
\end{aligned}
$$

When a reference beam is modulated in phase in one dimension, as shown in Fig. 19, it consists of wave vectors spanning a plane. We choose a spacing of plane wave components such that the discrete spectrum of wave vectors $\left(\boldsymbol{\rho}_{j}\right)$ corresponds to the wave vectors optimized for angular multiplexing. For proper selection of geometries, Bragg mismatch can be neglected. For this case, Krasnov has shown that the diffracted signal is given by [44]

$$
d_{c} \propto \sum_{l}^{N} \sum_{j}^{N} R_{l j}^{*} R_{c j} S_{l}
$$

in which the proportionality constant depends on the properties of the holographic material. For this special case, $\sum_{j}^{N} R_{l j}^{*} R_{c j}$ defines the inner product between $R_{l}$ and $R_{c}$. To minimize crosstalk, we require that the reference waves be orthogonal. In a practical application of phase encoding, we will typically have a configuration in which Bragg mismatch cannot be completely neglected; a more detailed analysis is required, but we can use the expressions obtained for $d_{c}$ above to place boundaries on the expected behavior of the system. Overall, we expect the crosstalk-limited SNR of an orthogonal phase-encoded multiplexing system to be comparable to the case of angular multiplexing, and somewhat higher as we deviate from strict orthogonality.

For a 2-D reference beam, the presence of Bragg selectivity reduces crosstalk due to Bragg mismatch, but crosstalk due to Bragg degeneracy cannot be eliminated. Generally, we desire a set of reference codes $r_{l}$ such that the autocorrelation functions closely approximate a delta function centered at zero, and that every pair of codes has a cross correlation that is uniformly small and ideally zero. Then the reconstructed signal will have minimal crosstalk.

When an SLM or phase diffuser generating the reference beam in the Fourier transform geometry spans two dimensions, significant crosstalk occurs due to Bragg degeneracies. Generally, the phase can be assumed to be random for each pixel, or wave vector address in the Fourier geometry, where a random phase varies uniformly from 0 to $2 \pi$ For 2-D random phase codes, crosstalk is estimated as [27]

$$
\mathrm{SNR}=\frac{M_{z}}{N}
$$

where $M_{z}$ is the number of resolvable reference beam wave vectors in the $z$ dimension

$$
M_{z}=\frac{2 n L}{\lambda} \sin \frac{\Theta}{2}
$$


The phase of each corresponding reference beam plane wave component is individually determined.

For the architecture in Fig. 19 having a 2-D reference beam SLM, each pixel corresponds to a distinct reference beam wave vector. These distinct reference beam wave vectors may be referred to as degrees of freedom [27], [28]. Usable SNR can, therefore, be obtained by having more degrees of freedom in the reference beam than number of holograms, or oversampling the reference beam. For example, if a speckle pattern is defined by illuminating a phase plate at the front focal plane of a lens, SNR generally improves as the number of grains increases, up to a saturation limit.

Recent advances in phase-encoded multiplexing are described in [45]. An important feature of phase-encoded multiplexing not afforded other multiplexing approaches is encryption of recorded data using phase encoding. Ideally, the capacity of phase-encoded multiplexing is comparable to that of angular multiplexing. Demonstrations and practical implementations, however, have been limited by the suitability of available phase-encoding SLMs.

Correlation Multiplexing: For the holographic disk geometry, shift multiplexing is the most suitable and natural solution, since it does not require any beam steering or wavelength change and is achieved by simply moving the medium with respect to the reference and object beams, as indicated in Fig. 2. Shift multiplexing can be implemented by using a spherical wave [46] or, alternatively, a random or, pseudorandom speckle pattern as a reference beam [12]. Shift multiplexing in the spherical reference beam case relies on the fact that a spherical type beam contains multiple angular components and, thus, a shift of the media or the reference beam leads to conventional Bragg mismatch. Multiplexing with a speckle beam is based on the spatial autocorrelation function of the beam used for the recording. In such case, the diffraction efficiency of the hologram is determined by the degree of correlation between the speckle beam used for the recording and the speckled beam presented upon readout (e.g., a spatially shifted version of the original beam). For random Gaussian speckle, the hologram shift selectivity is determined by the beam autocorrelation function [12]

$$
\eta(\delta)=\eta(0)\left|\frac{2 J_{1}(k \mathrm{NA} \delta)}{k \mathrm{NA} \delta}\right|^{2}
$$

where $\delta$ is the shift amount, $k=2 \pi / \lambda$, NA is the numerical aperture of the reference beam, and $J_{1}$ is the first-order Bessel function of the first kind. The speckle selectivity does not depend on the direction of the media shift (Bragg selective or Bragg degenerate) and is independent of the media thickness, although the crosstalk buildup is usually much faster in the Bragg-degenerate direction (and in thin media).

Thin-film-based materials are not typically suitable for angular multiplexing due to the difficulty in achieving the perpendicular geometry.

Qualitatively, speckle multiplexing can be modeled using the formalism developed for phase-encoded multiplexing described above. The speckle pattern will produce a plane wave spectrum that will not generally have optimum Bragg selectivity properties. Rather, the cross-correlation formulation is a better model to describe the process, and in general a mixed approach is needed to develop a complete qualitative analysis [27]. In general, the shift multiplexing selectivity is equal to the autocorrelation length of the speckle field, which is $1.2 \lambda / \mathrm{NA}$ in both the $x$ and $y$ directions.

The details of the crosstalk will depend on the details of the speckle pattern and the architecture of the HDSS. Crosstalk decreases as the number of degrees of freedom associated with the speckle pattern $M_{z}$ increases.

In this geometry, the number of accessible data channels can be approximately estimated as

$$
Q=\frac{8 V}{\lambda^{3}}(\mathrm{NA})^{2}\left(\cos \theta_{1}-\cos \theta_{2}\right)
$$

where NA is the numerical aperture of a signal incident at surface normal and the angles $\theta_{1}$ and $\theta_{2}$ are the minimum and maximum incident angles of the wave vector spectrum of the speckle field of the reference beam, in free space outside the material. The randomness of the speckle pattern generally results in a number of accessible data channels significantly less than this upper limit.

While the details of correlation multiplexing are more complex than random phase-encoded multiplexing described above, it is useful to compare the two to understand the crosstalk trends of correlation multiplexing. In general, the details of the crosstalk will depend on the details of the speckle pattern and the architecture of the HDSS. We can refer to (41) to estimate the upper bound of SNR. In particular, (41) shows that crosstalk decreases as the number of resolvable reference beam wave vectors in the $z$ dimension associated with the speckle pattern $M_{z}$ increases. For speckle multiplexing geometries, $M_{z}$ is given by

$$
M_{z}=\frac{2 n L}{\lambda}\left(\cos \theta_{1}-\cos \theta_{2}\right) .
$$

By engineering the magnitude and the correlation function of the phase mask, the decorrelation distance is modified. Excellent densities can be achieved with this method, as shown in Fig. 21.

With the random phase mask in the reference beam, 100 holograms were superimposed with good diffraction efficiency, as shown in Fig. 22. These results indicate that densities of over $60 \mathrm{~b} / \mu \mathrm{m} 2$ can be achieved with this technique.

Shown in Fig. 23 is an example of multiplexing multiple holograms within virtually the same volume of the medium using this technique.

In the Stanford HDSS holographic disk system described below, with the medium thickness of $200 \mu \mathrm{m}$, the hologram shift selectivity is approximately $\pm 1.0 \mu \mathrm{m}$ and the corresponding NA of the reference beam is $\sim 0.25$.

\section{E. Phase-Conjugate Readout}

An important approach for readout of holographic data is to use a phase-conjugate reference wave. A hologram is recorded in a typical manner, as shown in Fig. 24(a). In 


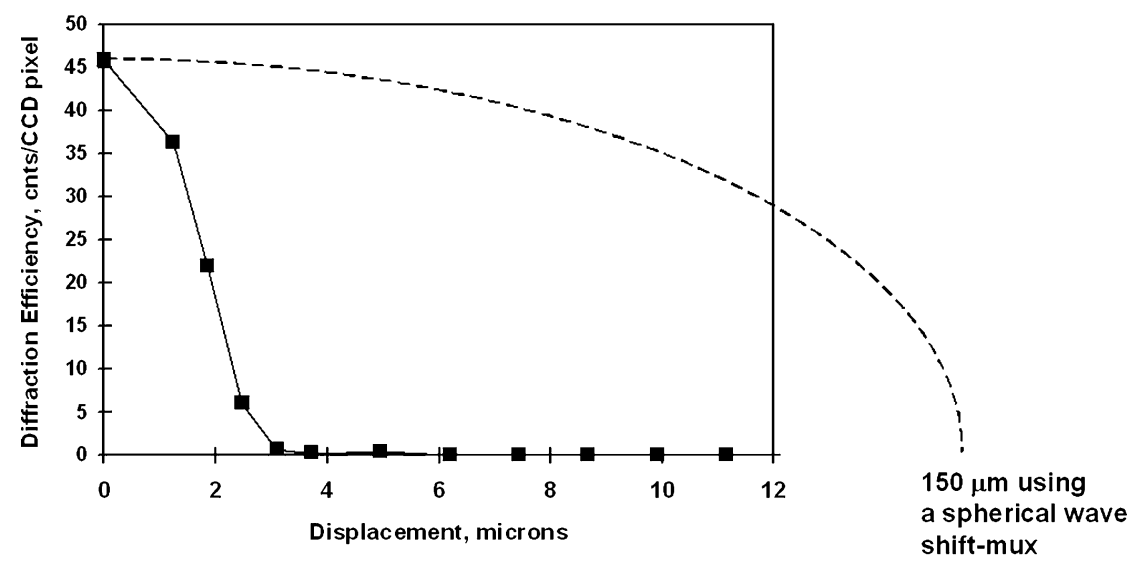

Fig. 21. Spherical wave and random phase mask multiplexing results for $200-\mu \mathrm{m}$-thick sample of Polaroid photopolymer ULSH-500.
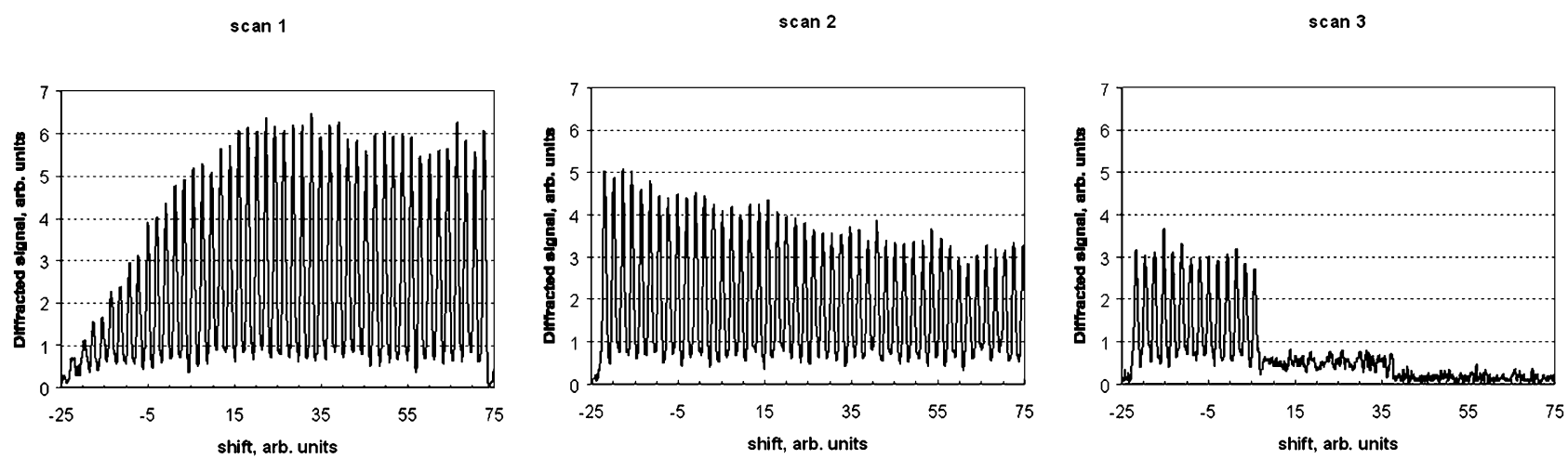

Fig. 22. Superposition of 100 holograms without implementation of scheduling.

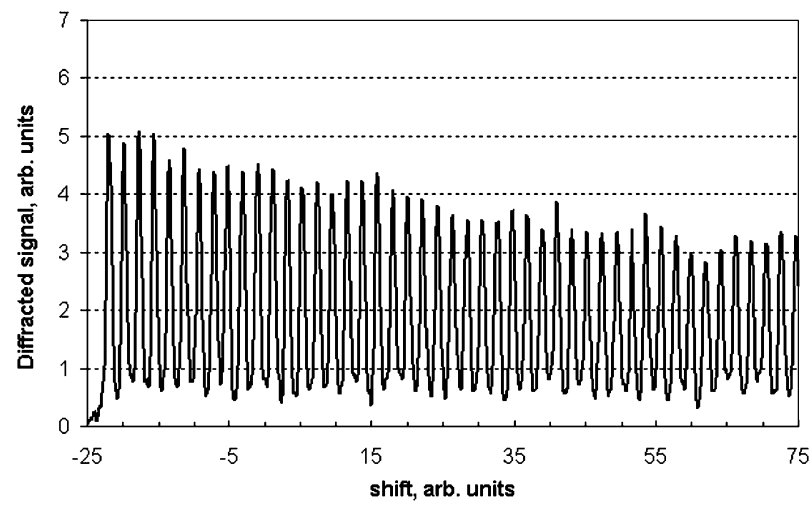

Fig. 23. Holograms superimposed using speckle shift multiplexing (sample out of 140). NA of the speckle reference is 0.2 ; media thickness is $200 \mu \mathrm{m}$.

a phase-conjugate readout configuration, a counterpropagating phase-conjugate reference beam is incident on the material, as shown in Fig. 24(b). This reference beam generates a phase conjugate of the signal beam, which reverses the distortions in the material. Crosstalk between multiplexed holograms and noise caused by strong distortions in a holographic storage material can be reduced by readout with a phase-conjugated reference wave. Such a scheme has been suggested by several authors [47], [48] and implemented in

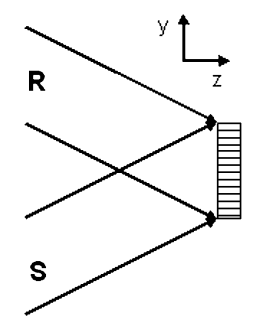

(a)

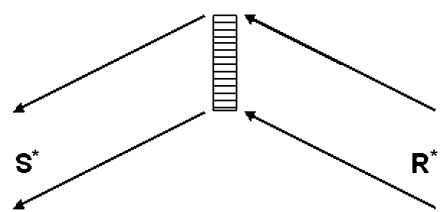

(b)
Fig. 24. Holographic optics processes for: (a) recording and (b) phase-conjugate readout.

simple proof-of-concept demonstrations for materials displaying strong scattering [49]-[51]. More recently, digital HDSSs using phase-conjugated readout have been evaluated at Caltech [9], [52] and IBM [1].

Phase-conjugate readout, although being an extremely elegant technique realizable with inexpensive optical components, puts severe limitations on the performance of the system in terms of media and channel evaluation capabilities, since direct imaging (without writing a hologram) is not possible. Second, the phase-conjugation technique requires (in most cases) the capability of generating a phase-conjugate (or pseudophase-conjugate) replica of the reference beam to be presented during readout, which is rather cumbersome 


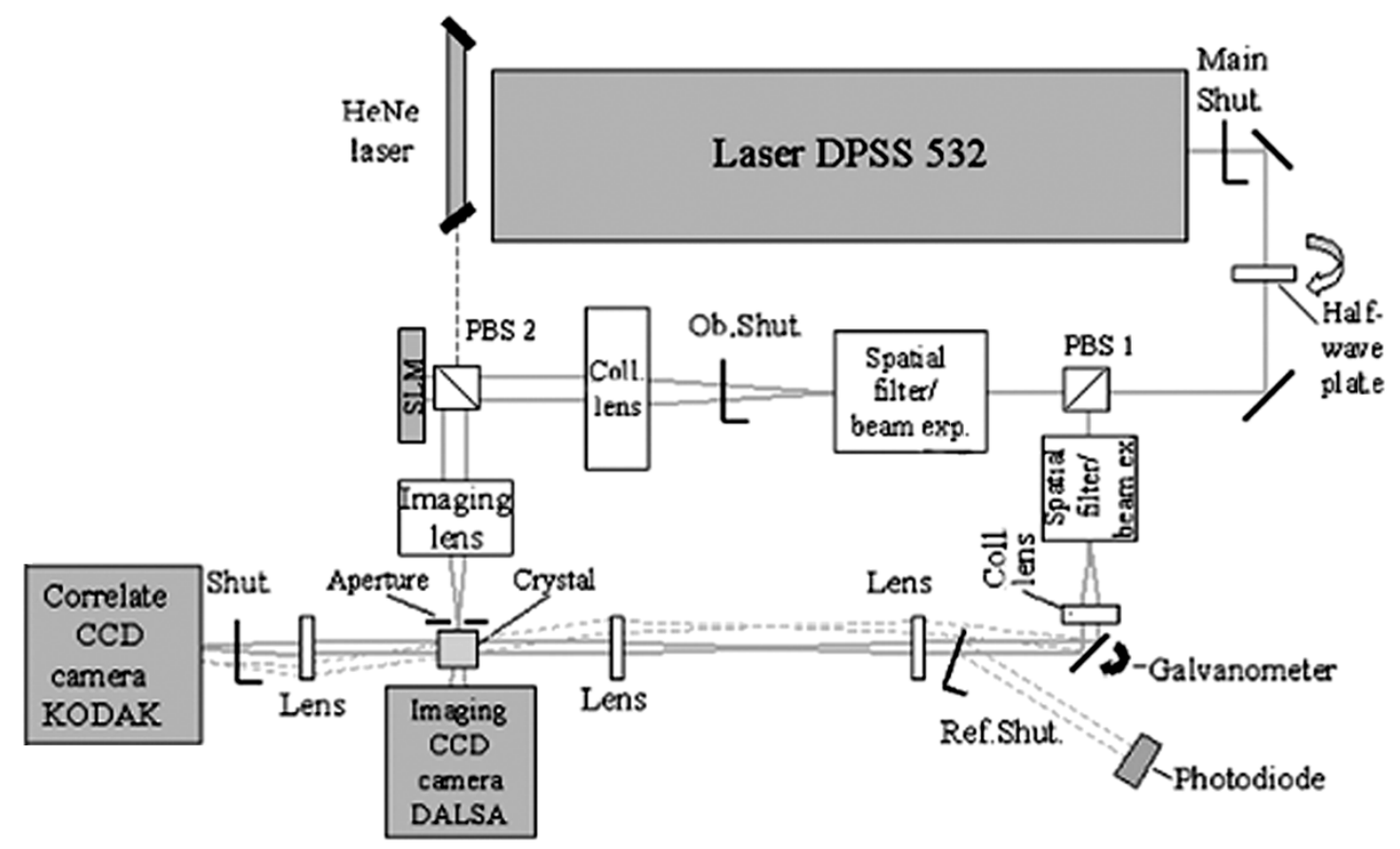

Fig. 25. Layout of the holographic search test bed. The diode-pumped YAG laser (DPSS 532) is used to record/read/search holograms. The HeNe laser is used for alignment.

to implement within a shift multiplexing geometry using a speckle or phase coded reference beam. An alternative approach employs storage of a phase-conjugated replica of the reference beam itself using a buffer hologram [53].

\section{Associative SEARCH}

Volume HDSSs can be used to perform fast data searches of the stored data pages. Because the readout of each hologram multiplies two 2-D Fourier transforms (the transform of the search argument and the 2-D Fourier transform of the stored images), multiple 2-D correlations are computed optically. When multiple volume holograms are presented under the SLM with the search argument presented on it, the reconstructed reference beams are generated, with their respective amplitudes being proportional to the correlation strength between the search argument and the stored data pages. The search speed is basically limited by the data recording density and, for the system described below, can be as high as $10-100 \mathrm{~Gb} / \mathrm{s}$.

Volumetric holograms exhibit substantial Bragg selectivity with respect to small angular changes of the readout beams in the Bragg-selective direction. Thus, if the search pattern (search argument) is shifted with respect to its position in one of the stored images, the reconstructed reference correlation signal will be substantially reduced due to Bragg mismatch. In the Bragg-degenerate direction, the Bragg selectivity itself is a lot more forgiving, but the reconstructed reference signal will be angularly displaced in the Bragg-degenerate direction by an amount proportional to the relative shift between the search argument and its position in the given stored image. The shifted reconstructed reference beams can be filtered optically (by a pinhole and the correlation CCD pixel itself) before correlation signal detection in order to increase the autocorrelation to cross-correlation ratio.

Due to the lack of shift invariance, volume holographic optical correlators are most suited for relational database searches in which each individual data field has a fixed assigned position within the recorded hologram [54].

The holographic associative search testbed developed at Stanford has the capability to record and retrieve multiple holograms recorded in a $\mathrm{LiNbO}_{3}$ crystal by angular multiplexing, as shown in Fig. 25. The correlation of a group of holograms with an arbitrary search key can be detected with the correlation CCD camera. The control software allows the hologram(s) corresponding to the most intense correlation peaks to be selected.

Multiple holograms are recorded in the $\mathrm{LiNbO}_{3}$ crystal using the $90^{\circ}$ geometry by deflecting a galvanometer mirror in the reference beam path over different angles. The light source used initially was a diode pumped continuous-wave (CW) laser with nominal power of $400 \mathrm{~mW}$ and a wavelength of $\lambda=532 \mathrm{~nm}$. A twisted nematic liquid-crystal SLM (1024 $\times 1024$ pixels, pixel size $15.6 \times 15.6 \mu \mathrm{m}$, video frame rate), developed by IBM for the HDSS program was used as the page composer. The data readout CCD camera was manufactured by Dalsa, Waterloo, ON, Canada $(1024 \times 1024$ pixels, pixel size $12 \times 12 \mu \mathrm{m})$. One-to-one imaging is accomplished with a high-quality imaging lens built by the Rochester Photonics Corporation, Rochester, NY, for the HDSS consortium.

To read out the correlation field intensity measured, a Kodak Megaplus CCD camera is used, having $1008(\mathrm{H}) \times$ $(1018(\mathrm{~V})$ pixels, of $9 \times 9 \mu \mathrm{m}$ pixel size, and a center-to-center spacing of $9 \mu \mathrm{m}$ (20\% fill ratio). We used a pair of F-theta scan lenses, $F=100 \mathrm{~mm}$, scan angle $\pm 25^{\circ}$, spot diameter $12 \mu \mathrm{m}$ (Fig. 25), to obtain a small, near diffraction limited 


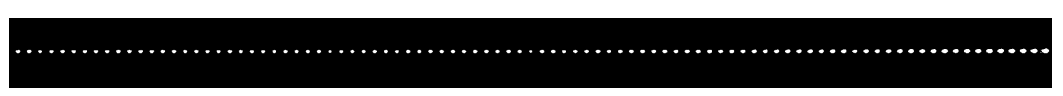

Fig. 26. Example of correlation field captured by Kodak CCD camera; 100 holograms are recorded.

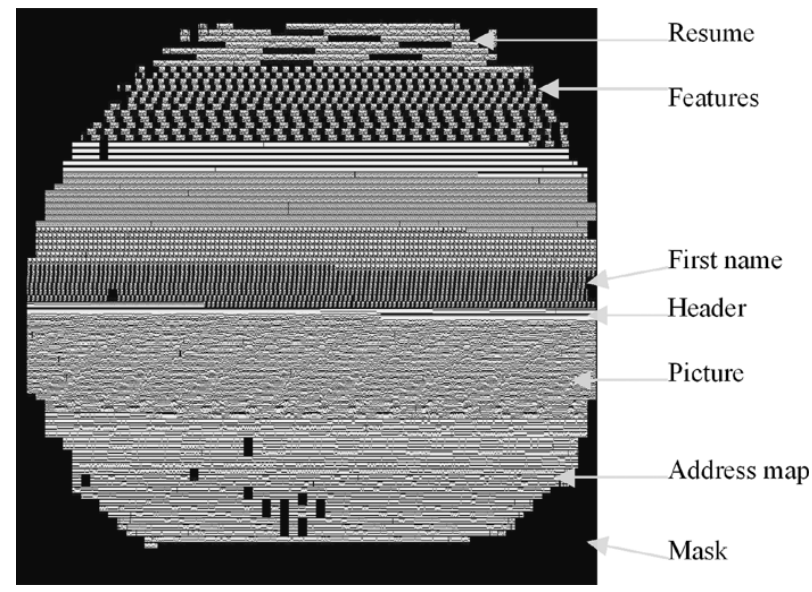

Fig. 27. An example bitmap image from the demo database. The bitmap image can be displayed on the SLM for recording or searching. A mask has been used to eliminate areas of poor image quality from use. Each database field has a fixed position in the bitmap image. The holographic image contains personal information of a person stored in the database.

correlation spot size for all holograms within the reference beam angular range. To minimize crosstalk and optimize overall system performance, the spacing between correlation spots is typically increased by decreasing the number of recorded holograms to 334 - using three pixels per correlation period.

Fig. 26 shows an example of the correlation field with the scanning lens reference beam telescope ( 100 holograms were recorded).

The experimental system was used to demonstrate fast parallel searches in a relational database. A relational database has data organizational structure such that the specific data is located in a specific part of the stored holographic page, thus eliminating the difficulties associated with holographic shift invariance. In the Stanford system, the demo database is a relational database that contains personal information about people. A typical data encoded page used in the demonstration is shown in Fig. 27.

To demonstrate the parallel search ability of a volume holographic associative memory, 200 holograms were stored in $90^{\circ}$-geometry in $\mathrm{Fe}: \mathrm{LiNbO}_{3}$ crystal in the associative search testbed [55]. Each hologram represents one record from the demo database. The reference beam angles were chosen in such a way that each reference beam was focused to a unique portion of the correlation camera. An experimentally verified exposure schedule was used to achieve a uniform diffraction efficiency distribution among the multiplexed holograms. The processing software converts the user query inputs into a search key bitmap file by using appropriate modulation coding and encoding mask. Shown in Fig. 27 is an example search key bitmap file.

After the search keys is presented on the SLM, the image of the correlation spots is grabbed by the correlation CCD

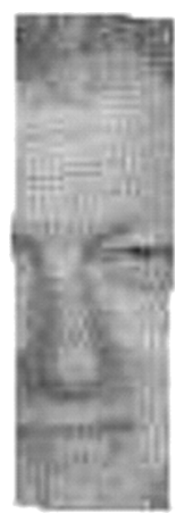

Fig. 28. Query picture. 80\% of the original photograph has been erased.

and, after applying normalization to the correlation spot intensities, the processing software ranks all the correlation spots in the order of the correlation intensity values. An example of search system operation is presented in Figs. 28 and 29.

The performance of the volume holographic associative memory was evaluated in a number of search demonstrations. When any one of the ten database fields was used as the query key, the expected records were consistently within the top ten of the 200 search results. With a larger query key (for example, using the combination of the first name and last name field as the query key) better search results were obtained. Fig. 28 is a partial picture that we used as the query key. Eighty percent of the photo in the query key has been erased, but it still returns the correct record. The retrieved complete personal record (Fig. 29) is identical to the original record.

Each search operation includes the following: imprinting the search key on the object beam and illuminating stored holograms; detection of the correlation signal, normalization by hologram strength, ranking of the correlation intensity, mapping of selected correlation spots to reference beam angles, address-based recall of the selected hologram, decoding of the retrieved data page, and finally, display of the search result.

In the current system, the major time overhead is associated with mechanical components, signal processing, and search argument display. The fundamental limit to the search speed comes from the key generation and correlation signal processing (ranking and normalization). With dedicated hardware and a fast SLM [such as a 1000-ft/s Texas Instruments deformable mirror device (DMD)], search speeds of 10000 stored pages/s or more can be envisaged.

At IBM, a similar associative search experiment was carried out (see Fig. 30) [1]. Researchers at IBM showed that if an unindexed conventional "retrieve-from-disk-and-compare" software-based database is searched, the search is limited by the sustained hard-disk readout rate (taken to be 


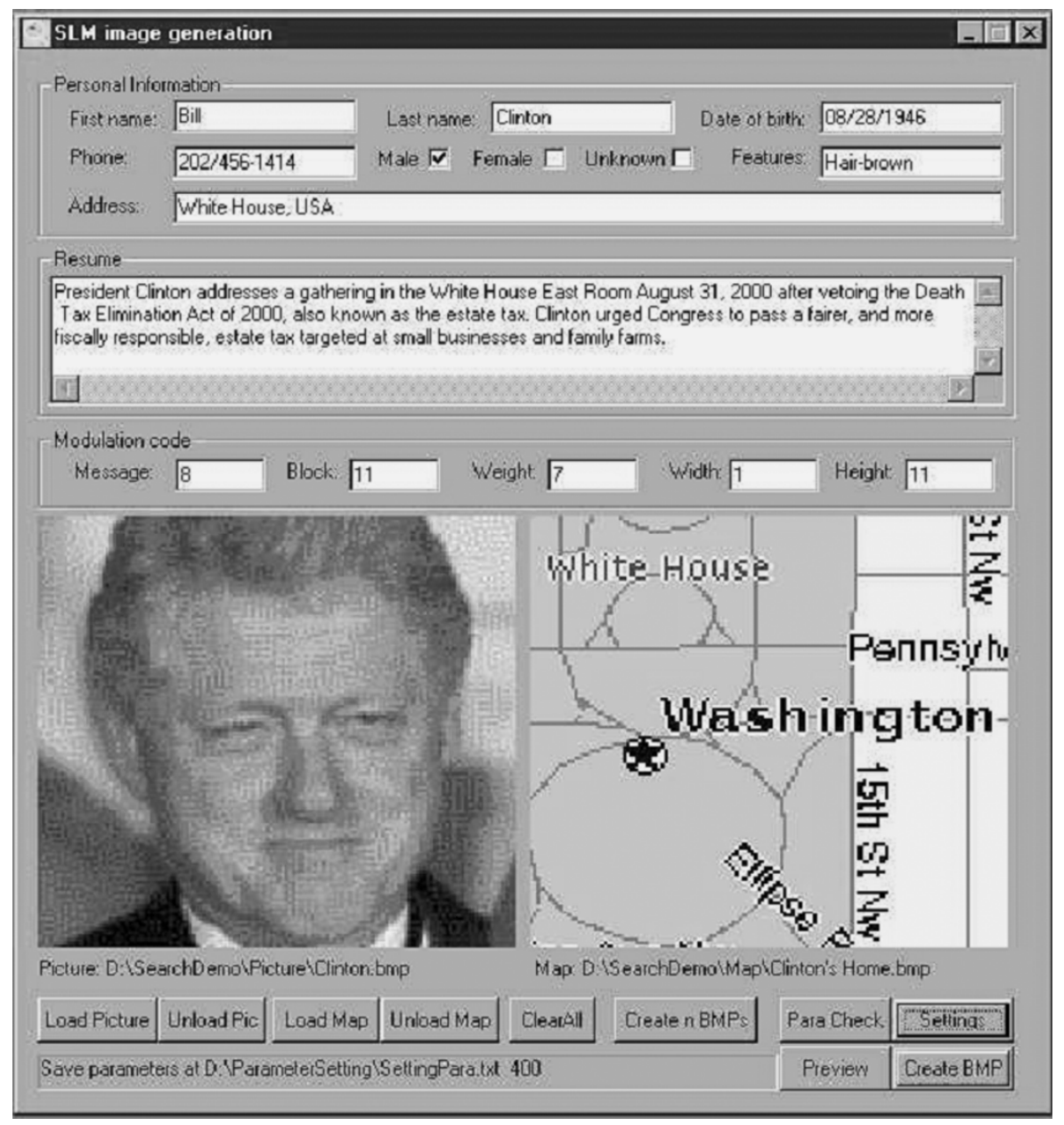

Fig. 29. The first ranked search result. While using pictures as the query key, the volume holographic associative memory is in fact doing pattern recognition.

$25 \mathrm{MB} / \mathrm{s})$. For example, a search over 1 million 1-kB records would take $\sim 40 \mathrm{~s}$. In comparison, with off-the-shelf, videorate SLM and CCD technology, an appropriately designed holographic system could search the same records in $\sim 30 \mathrm{~ms}$, a $1200 \times$ improvement. Custom components could enable 1000 or more parallel searches per second.

\section{HOLOGRAPHIC MATERIAL CHARACTERISTICS FOR Digital DATA STORAGE}

Although different applications require materials of varying properties, an ideal material would have a fast time response (in microseconds), would be as as sensitive as photographic film (in joules per square centimeter), would retain information over long periods ( $>$ ten years), would have a large spectral sensitivity range extending into the infrared region of the spectrum, and would be available in large quantities and sizes with good optical quality, and would cost little. Such a recording material does not exist today. In general, sensitive materials have small photorefractive response, and vice versa. For example, $\mathrm{LiNbO}_{3}$ is available in large size, of good optical quality, and at relatively low cost, and has a large index variation per absorbed photon. Its response time, however, is rather slow, on the order of milliseconds or longer, depending on the intensity of the recording light. Thermal fixing has been demonstrated allowing nondestructive readout for periods of years, but this process typically requires temperatures of at least $100{ }^{\circ} \mathrm{C}$ for fixing.

Photopolymer materials usually have much higher sensitivity due to chemical amplification effects and a high dynamic range, but are rather limited in terms of thickness (1 $\mathrm{mm}$ or less). Other issues associated with polymers are significantly increased scatter levels compared to single-crystal photorefractive materials, and volumetric shrinkage upon photopolymerization. The latter effect can be somewhat mitigated by use of high molecular weight monomers and proper choice of the material chemistry. The shrinkage and scatter limits the available usable thickness of photopolymers.

The typical minimum material parameters for optimized holographic performance are listed below [56].

Material:

- Thickness: $L>0.5 \mathrm{~mm}$.

- Sensitivity: $S>500 \mathrm{~cm} / \mathrm{J}$ (or $\mathrm{SL}>20 \mathrm{~cm}^{2} / \mathrm{J}$ ).

- Dynamic range: $(M \#)_{\text {planewave }}>5.0$ (or, $\left.(M \#)_{\text {image }}>2.0\right)$.

- Shrinkage: $<0.05 \%$ (for $500 \mu \mathrm{m}$ ).

- Scatter: $<10^{-5} \operatorname{srad}^{-1}$. 


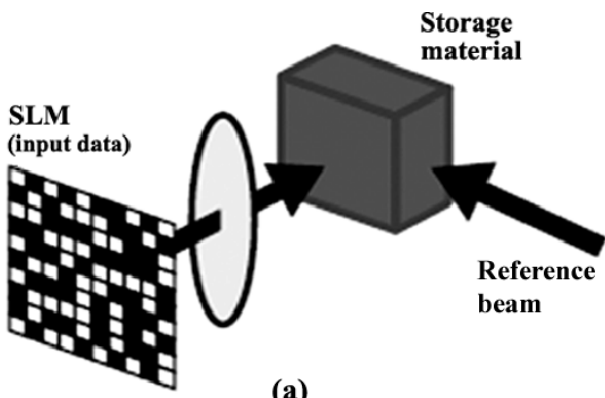

(a)

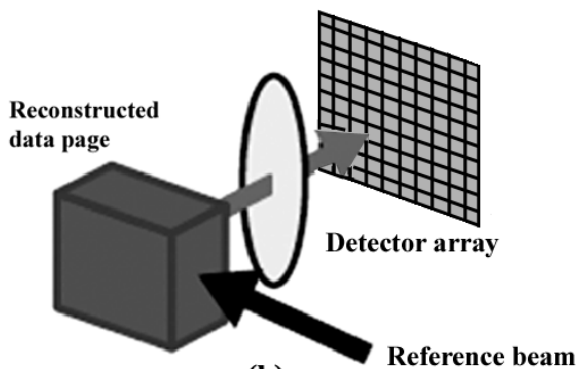

(b)

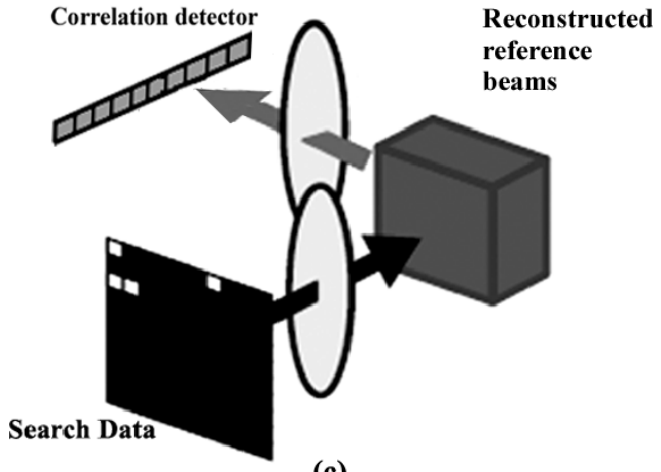

(c)

Fig. 30. The top two images show schematically the holographic storage of a page of data, while in the bottom picture, the retrieval of the page address is achieved among all stored pages by using a search key as input to the system. The page address is measured with a linear detector array (Courtesy of IBM, with permission).

— Wavelength response: visible ( $532 \mathrm{~nm}$ or blue).

\section{Media:}

- Disk wedge $<0.5 \mathrm{mrad}$.

- Format: $120 \mathrm{~mm}$ to 6.5-in-diameter disk.

- Total wavefront error over $\sim 2 \mathrm{~mm}$ spot: $<\lambda / 10$.

- Scatter (including substrate) $<10^{-5} \mathrm{srad}^{-1}$.

The performance of an HDSS greatly depends on the quality and physical properties of the recording medium. The DARPA PRISM consortium has developed a precision tester [57] at IBM for measuring the performance of different holographic media using a common platform. Since 1996, many advanced holographic recording materials were evaluated and compared using a common comparison framework. The most important parameters include image quality (degree of image distortion), sensitivity, dynamic range, fidelity (spatial resolution), stability, shrinkage, and available thickness. Based on the results published so far [58], the most promising photorefractive crystals remain Fe-doped lithium niobate, while Polaroid CROP materials represent the better option in the photopolymer family.
Fig. 31 presents the summary of current holographic storage materials properties (data collected at the International Workshop on Holographic Data Storage, sponsored by NSIC, Nice, France, March 8-11, 1999).

The PRISM and HDSS team members have investigated the properties of many available materials in the IBM PRISM tester. The results are displayed in Fig. 32 The BER was measured for a given material as a function of background scatter, as defined above. It is notable that $\mathrm{LiNbO}_{3}$ is still the best material in terms of optical quality at a certain BER at all page sizes. This is not surprising, as the polymer materials intrinsically scatter more light caused by the variation in index of refraction of the components that constitute the polymer. These components, such as the binder, the monomer and the polymer are needed to create a hologram; therefore, a tradeoff is required between sensitivity, diffraction efficiency and scatter. For inorganic materials such as $\mathrm{LiNbO}_{3}$ the index of refraction is induced by a space charge field and scatter (as well as sensitivity) is considerably lower. Currently Aprilis and InPhase are optimizing their materials for improved performance. From this graph we also note that the page size has a considerable effect on the achievable BER. For a page that contains $1024 \mathrm{~kb}$, the achievable BER is six orders of magnitude worse than for a $256-\mathrm{kb}$ page, while reducing by only a factor of four the capacity per page. To optimize total system capacity, a careful tradeoff is needed between page size and the multiplexed number of pages. For example, by setting a threshold value for the minimum BER, we can determine the optimal capacity by considering the number of pages that can be superimposed using a certain error correction code (ECC), as described in the section on en/deconding.

\section{Photorefractive Materials}

Photorefractive materials record holograms through photoinduced charge-transport (diffusion, drift, and photovoltaic effect), charge carrier redistribution between the deep traps and buildup of an internal space charge field. The resulting index distribution is produced due to electrooptic (Pockels) effect (Fig. 34).

In general a variety of trapped and photoexcited states are possible, including electrons and holes. Typically, electrons are trapped at impurity centers and photoexcited into the conduction band. This example is illustrated as follows. Using the example of Fig. 4, intersecting signal and reference beams generate an interference pattern with regions of high intensity and low intensity, which has the functional form

$$
I=I_{0}(1+m \cos K y)
$$

in which $m$ is the modulation factor that quantifies the contrast of the interference pattern.

As shown in Fig. 33, photons excite electrons into the conduction band in higher concentrations within the regions of constructive interference than within the regions of destructive interference. Electrons diffuse in the direction of the concentration gradient to the regions of destructive interference, where they relax to empty states in the energy band gap. In 


\begin{tabular}{|c|c|c|c|c|c|c|c|}
\hline Material & $\begin{array}{c}\text { Sensitivity, } \\
\mathrm{cm} / \mathrm{J}\end{array}$ & $\begin{array}{c}\text { Thickness, } \\
\text { mm }\end{array}$ & $\mathrm{M} / \#$ & $\Delta_{n}, \max$ & $\begin{array}{c}\text { Scatter } \\
\text { srad }^{-1}\end{array}$ & $\underset{\mathrm{nm}}{\lambda, \text { range}}$ & Shrinkage \\
\hline $\mathrm{Fe}: \mathrm{LiNbO}_{3}$ & $0.01-0.1$ & $1-20$ & $10-20$ & $310^{-3}$ & $\begin{array}{c}110^{-7} \\
\text { (90 degree) }\end{array}$ & $400-600$ & -- \\
\hline $\begin{array}{l}\mathrm{LiNbO}_{3} \\
\text { two-photon } \\
\text { undoped }\end{array}$ & $\begin{array}{c}0.03 \\
\text { (trans.) }\end{array}$ & $1-10$ & 10 & $510^{-4}$ & $\begin{array}{c}110^{-7} \\
\text { (90 degree) }\end{array}$ & $650-900$ & -- \\
\hline $\begin{array}{l}\mathrm{LiNbO}_{3} \\
\text { two-photon } \\
\text { Fe-doped }\end{array}$ & $\begin{array}{c}0.04 \\
\text { (trans.) }\end{array}$ & $1-10$ & 10 & $510^{-4}$ & $\begin{array}{c}110^{-7} \\
\text { (90 degree) }\end{array}$ & $650-900$ & -- \\
\hline $\begin{array}{c}\mathrm{KNbO}_{3} \\
\text { Fe-doped }\end{array}$ & 600 & $1-10$ & $1-2$ & $10^{-4}$ & $>\mathrm{LiNbO}_{3}$ & $400-600$ & -- \\
\hline PRP & $210^{4}$ & $0.01-0.05$ & - & $10^{-2}$ & $?$ & $480-830$ & $?$ \\
\hline Hetero-PAP & $0.1-100$ & $0.01-0.2$ & 1 & 0.1 & $?$ & $400-600$ & 0 \\
\hline $\begin{array}{l}\text { Polaroid } \\
\text { CROP }\end{array}$ & $5000-500$ & $0.2-0.5$ & $7-15$ & $510^{-3}$ & $110^{-3}$ & $480-540$ & $0.1 \%$ \\
\hline $\begin{array}{l}\text { Lucent } \\
\text { acrylate }\end{array}$ & $<500$ & 1 & $40 ?$ & $910^{-3}$ & $?$ & 500-532 & $0.35 \%$ \\
\hline $\begin{array}{l}\text { DuPont } \\
\text { HRF-150 }\end{array}$ & $\sim 4000$ & 0.038 & $0.5->1$ & $\begin{array}{l}610^{-3}- \\
2.810^{-3}\end{array}$ & $?$ & $400-600$ & $2.5-3 \%$ \\
\hline
\end{tabular}

Fig. 31. Summary of holographic materials properties.

the presence of a photovoltaic current found in materials such as lithium niobate, the effect is enhanced.

A graphical depiction of the relevant physical quantities associated with the photorefractive effect is shown in Fig. 34 for a diffusion dominated photorefractive medium. A periodic intensity variation $I$ arises from the interference of the signal and reference beams. This pattern establishes a coulombic charge density $\rho$ in the photorefractive material that records the intensity pattern; red indicates positive charge and blue indicates negative charge. At steady state, a periodic potential $U$ associated with the charge density balances diffusion. The separation of electrons from fixed, positive ions establishes an electric field $E$, typically called a space-charge field, that replicates the functional form of the interference pattern, up to a phase factor $\phi$

$$
E=\left|E_{\mathrm{SC}}\right| \cos (K y+\phi) .
$$

This expression is accurate only for small modulation depths, in which $E_{\mathrm{SC}}=m \mathcal{E}_{1}$ and $\mathcal{E}_{1}$ is the normalized space-charge field, representing the transport properties of the medium.

In materials displaying the linear electrooptic (Pockels) effect, for example, the space-charge field generates a periodic index grating $\delta n$ proportional to the electric field, such that

$$
\delta n=-\frac{1}{2} n^{3} r m \mathcal{E}_{1} \cos (K y+\phi)
$$

where $n$ is the index of refraction and $r$ is the effective Pockels coefficient, which in general depends on the Pockels tensor, light polarization, and crystal orientation [59]. Therefore, the resulting index grating replicates the initial interference pattern up to a phase shift, generating a volume hologram.

In a holographic medium that does not display nonlinear beam coupling, the diffraction efficiency is [60]

$$
\eta=\sin ^{2}\left(\frac{\pi m n^{3} r\left|\mathcal{E}_{1}\right|}{2 \lambda \cos \theta}\right) .
$$

Detailed accounts of photorefractive transport can be found in [61]-[66]. Lithium niobate is the most common photorefractive material used in HDSS demonstrations. Other inorganic materials of interest include strontium barium niobate and barium titanate. Organic materials include reversible photorefractive polymers that may eventually be candidates for holographic storage systems [67]. Photorefractive polymer materials, however, require large applied electric fields, and good sensitivity as well as long term stability of recorded holograms have not yet been demonstrated in the same medium.

\section{A. Complementary Gratings for Hologram Fixing}

In a simple photorefractive material with one photoexcitation process and one species of trapped charge, light used to record the hologram will typically erase the same hologram upon readout. Thus, for permanent storage and multiple reads without data degradation, photorefractive materials require a fixing process.

Hologram fixing can be accomplished in a number of ways. One method makes use of complementary charge gratings residing in separate subsystems of charge transport which are not sensitive to the readout light. The gratings are 


\section{OpticalQualityofHolographic StorageMaterials}

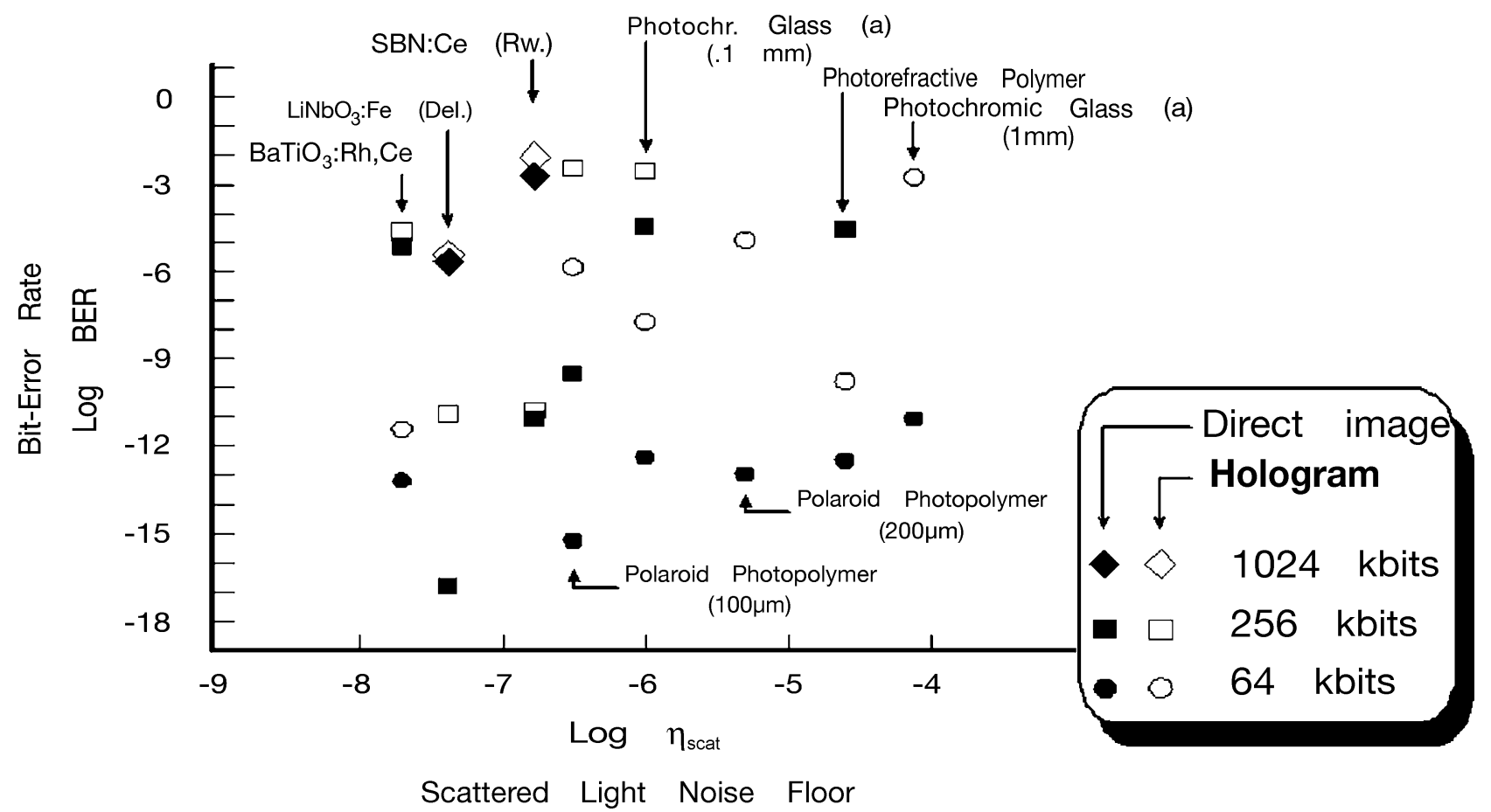

Fig. 32. Optical quality of holographic storage materials (courtesy of R. M. Shelby, IBM Almaden Research Center with permission [58].

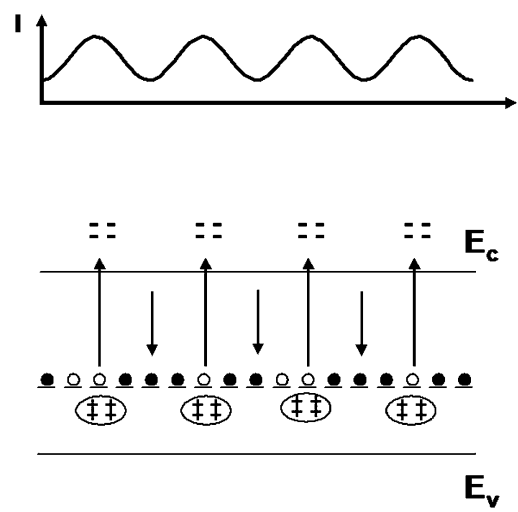

Fig. 33. Photoexcitation of electrons in the presence of a periodic intensity.

complementary in the sense that they have opposite polarity and can screen each other. Fig. 35 illustrates a generic process that uses complementary gratings to establish stable holograms. Subsystem $A$ comprises a photoexcitable charge species, such as a trapped electron, as shown in Fig. 35(a). A coulombic charge density grating $\rho_{A}$ is written in subsystem $A$ through the photorefractive effect. A second charge density grating $\rho_{B}$ is established in subsystem $B$ using a different process and may be accomplished by changing a material environment parameter such as temperature, electric field, or type of illumination, as shown in Fig. 35(b).

Optical, holographic, and system considerations put constraints on the use of complementary gratings for nonvolatile
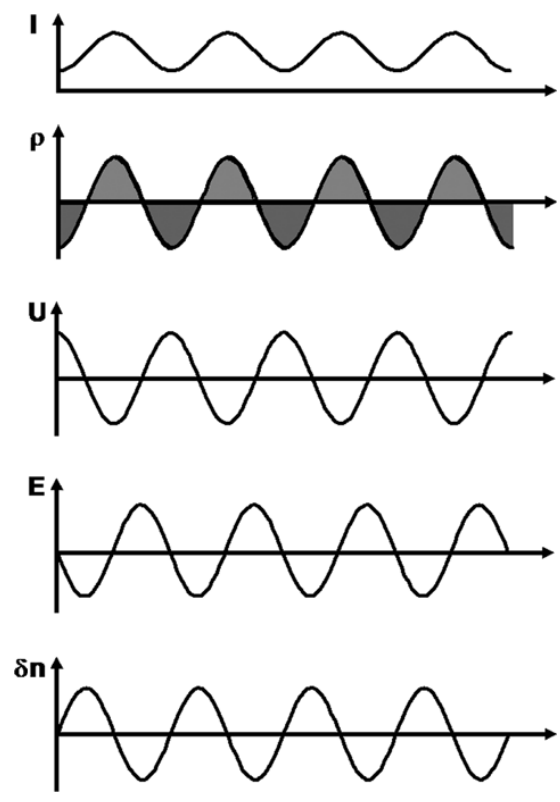

Fig. 34. Photorefractive effect in diffusion dominated materials.

readout. For image bearing beams, an important issue is the expansion of the crystal on heating. If holograms are recorded at an elevated temperature and then cooled, the Bragg condition will be modified, and either the angle of the reference beam or its wavelength or both must be modified to obtain the greatest diffraction efficiency. This effect ultimately puts limits on the image field that may be 

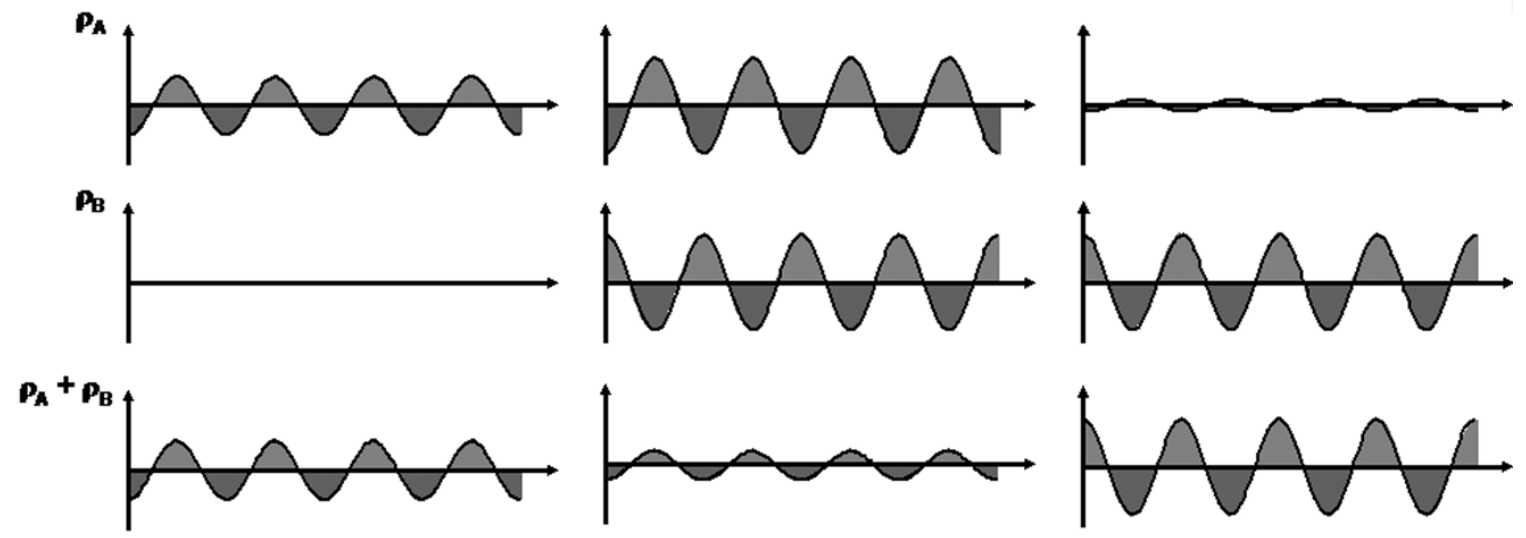

(b) Complementary Grating

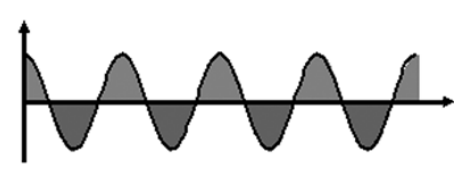

(c) Exposed Grating

Fig. 35. Graphical depiction of the evolution of complementary gratings, illustrating charge density grating $\rho_{A}$ in subsystem $A$, charge density grating $\rho_{B}$ in subsystem $B$, and the net grating $\rho_{A}+\rho_{B}$, for: (a) initial grating, (b) complementary gratings, and (c) exposed grating for readout.

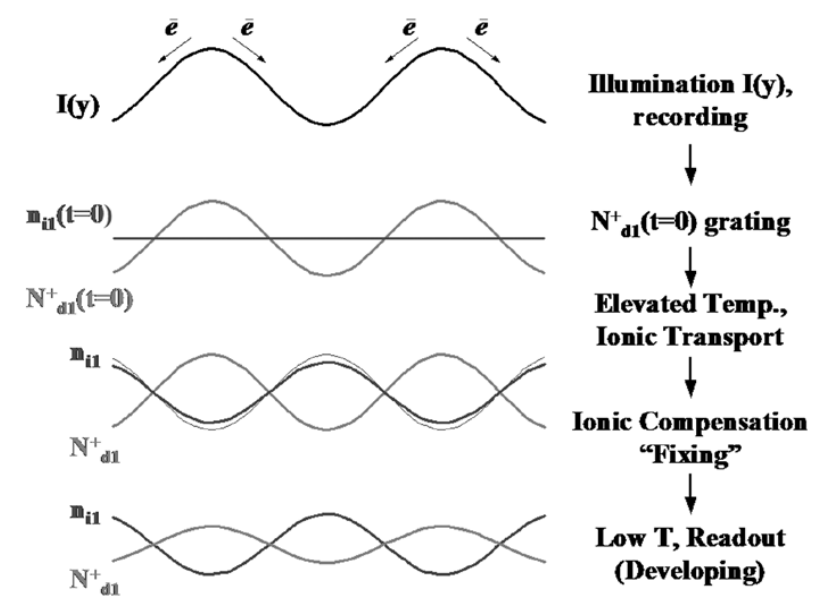

Fig. 36. Mechanisms of holographic fixing via thermally assisted ionic drift.

reconstructed with acceptable fidelity and will introduce additional crosstalk because not all of the signal can be Bragg matched simultaneously at the lower temperatures. It is a system issue whether simultaneous formation of holograms at high temperature or formation a low temperature and subsequent activation of mobile ions at high temperature give optimal performance.

In lithium niobate, for example, subsystem $A$ may establish an initial electronic charge density through the photorefractive effect, and subsystem $B$ is established as a complementary charge density through mobile ion transport at elevated temperature [68]-[71] (Fig. 36). The ionic conductivity usually obeys an Arrhenius-type dependence on the temperature $T$

$$
\sigma_{i}=e n_{i} \mu_{0} \exp \left(-\frac{E_{a}}{k_{b} T}\right)
$$

where $E_{a}$ is activation energy (approximately $1.2 \mathrm{eV}$ for hydrogen impurity and $\sim 1.4 \mathrm{eV}$ for interstitial lithium migration), $n_{i}$ is the density of conducting ions, $e$ is the electron charge, $\mu_{0}$ denotes the permeability of free space, and

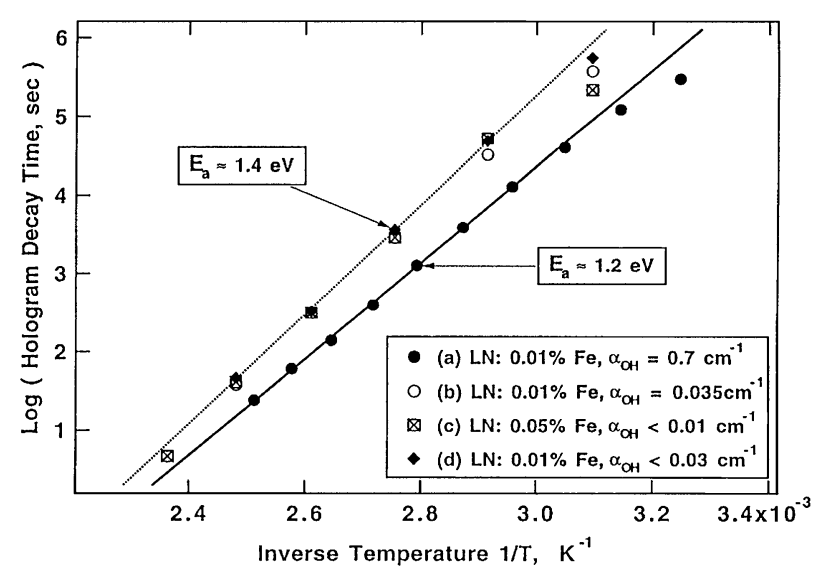

Fig. 37. Arrhenius plot of the ionic hologram decay time(unscreened) in: (a) as-grown crystal ( $E_{a}=1.2 \mathrm{eV}$, hydrogen impurity); (b) and (c) samples with low hydrogen impurity content and different Fe-doping ( $E_{a}=1.4 \mathrm{eV}$, lithium migration); and (d) near-stoichiometric $\left(\left[\mathrm{Li}_{2} \mathrm{O}\right]=49.5 \%\right) \mathrm{Fe}$-doped dehydrated crystal.

$k_{b}$ is Boltzmann constant. Mobile ions are thermally activated and move to screen out the electronic grating. The resulting hologram becomes screened. However, at lower temperatures, the ions have significantly lower mobility and the grating lifetimes can be considerably longer than for an electronic grating. Thus, at lower temperature, the ionic grating will represent a backbone of the fixed hologram while, upon light illumination, the electronic grating will redistribute itself, partially screening the fixed ionic grating. The ionic grating also eventually decays at a much slower rate (from several months to more than ten years) depending on the residual conductivity at the storage temperature and the degree of electronic screening.

Two alternative approaches exist for creation of fixed ionic gratings in lithium niobate. In the first case, electronic gratings are formed at room temperature. Initial exposure to the signal and reference beams generates an electronic grating which is followed by ionic compensation at higher temperatures without illumination. At elevated temperatures (above approximately $100{ }^{\circ} \mathrm{C}$ in $\mathrm{LiNbO}_{3}$ [72]), ions become 
active and generate a complementary screening grating that compensates the space charge arising from the electronic grating. These paired electronic and ionic gratings constitute complementary gratings and are derived from charge patterns with opposite polarity. On cooling to room temperature, the ions are significantly less mobile and represent the ionic backbone of the fixed grating. Subsequent light exposure reveals the fixed grating by partially redistributing the electronic charges. In the presence of light exposure, the ionic grating is partially screened, but in the case of $\mathrm{LiNbO}_{3}$, strong photovoltaic currents significantly reduces the screening. The actual degree of screening depends largely on the grating spacing, photorefractive impurity concentrations (e.g., $\mathrm{Fe}^{2+}$ ), and the reduction state of the material [66], [73].

In the second approach, both electronic and ionic gratings are formed simultaneously at an elevated temperature [68]. Simultaneous formation of electronic and ionic gratings typically results in stronger complementary gratings because of mutual screening during formation, in which the magnitudes of both gratings may be substantial, but their difference remains relatively small [69], [70]. The typically used temperature range for this process is $150-180^{\circ} \mathrm{C}$.

Multiple fixed holograms based on ionic gratings have been demonstrated in $\mathrm{LiNbO}_{3}$. [68], [74]-[76]. At least 500 holograms may be stored using ionic gratings and for which diffraction efficiencies in the range of $2-25 \%$ have been reported [68], [75], [76]. As part of the PRISM consortium, Siros Technologies first demonstrated fixed ionic gratings by recording over 500 pages of digital data in $\mathrm{LiNbO}_{3}$ [77]. Subsequently, the Psaltis group demonstrated fixing of thousands of holograms [78].

Holographic gratings based on charge redistribution inevitably decay due to ionic and electronic conduction. The lifetimes of fixed ionic holograms are limited by the finite ionic conductivity at low (i.e., room) temperature [66], [73] (Fig. 37). Ionic gratings are partially screened by trapped electrons upon readout (see Fig. 36), which decreases the resulting diffraction efficiency, but proportionally increases the grating lifetime. A significant increase in fixed ionic hologram lifetime is realized in lithium niobate with low hydrogen impurity content. Fixed hologram lifetimes of about two years at room temperature are projected in dehydrated lithium niobate crystals. Due to partial electronic compensation, the actual hologram lifetime is prolonged but at the expense of reconstruction efficiency. Prolonged recording at elevated temperature (in highly doped lithium niobate) usually results in a nearly fully compensated gratings with little or no electrooptic contribution, but with a sufficiently strong index perturbation arising from the photochromic effect due to substantial modulation in the dopant concentrations. Such gratings possess prolonged lifetimes (over 50 years or more) and do not require light for development.

\section{B. Two-Photon Recording}

One of the difficulties encounted with thermally assisted ionic fixing for digital hologram recording is that recording

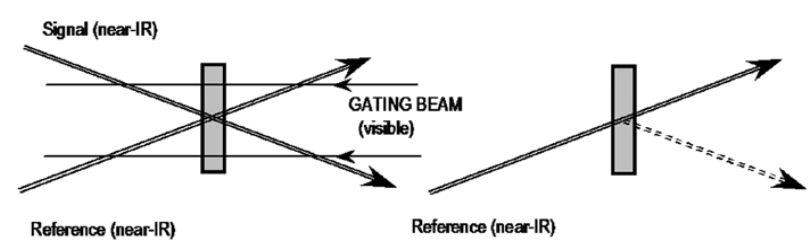

Fig. 38. Two-photon gated recording requires a visible incoherent gating beam in addition to two IR recording beams. During readout, only the IR reference beam is present. The light-induced IR sensitivity is about 100 times higher than without the gating beam, giving rise to prolonged readout.

occurs at elevated temperatures with reading at room temperature. For thick media, the diffraction efficiency, therefore, reduces due to Bragg mismatch. This problem and the associated complications of recording at elevated temperatures can be overcome by using a two-photon recording method.

Two-photon gated recording techniques (Fig. 38) have been investigated since the early days of research on holographic memories, in particular at Bell Laboratories [79] [80] and the PRISM consortium [81]-[84].

A signal and reference beam are incident at one (typical near IR) wavelength, which is too long for photoexcitation, and a uniform beam at a shorter wavelength provides the electron photoexcitation (and absorption at longer wavelength due to short-lived shallow traps). The gratings may then be probed nondestructively using the first wavelength. This process has been identified in nominally pure Fe-doped, and $\mathrm{Cu}$-doped $\mathrm{LiNbO}_{3}$ [80], [85]-[87], in $\mathrm{KTa}_{x} \mathrm{Nb}_{1-x} \mathrm{O}_{3}$ [79], and in $\mathrm{LiTaO}_{3}$ [80]. This process is nonlinear with intensity, and for optimal results, a resonant two-photon absorption process is desired. Nonresonant gratings require energy fluxes on the order of joules per square centimeter and peak pulse intensities of gigawatts per square centimeter, whereas resonant gratings require the same energy fluxes but peak pulses intensities of only $10 \mathrm{MW} / \mathrm{cm}^{2}$ [80]. Other work has focused on gated recording using rare earth doped ferroelectrics [81], [82]

The difficulty with the early work was the low sensitivity of the medium and the high power of the lasers required to record data in $\mathrm{LiNbO}_{3}$. In the late 1990s, we have overcome this problem by modifying the composition of $\mathrm{LiNbO}_{3}$ by making it more stoichiometric, adding $\mathrm{Fe}$ and other dopants, combined with optimized oxidation and reduction postgrowth treatments [83], [84]. As a result, we have discovered an improvement of sensitivity of several orders of magnitude over previous results, making the medium approximately equally sensitive as $\mathrm{Fe}$-doped $\mathrm{LiNbO}_{3}$ in the green. Typical sensitivity and index changes as a function of stoichiometry are shown in Fig. 39. It is interesting to note that the sensitivity exhibits a threshold behavior near 49.5 mol $\% \mathrm{Li}_{2} \mathrm{O}$ in the crystal.

At a $\mathrm{Li}_{2} \mathrm{O}$ concentration of $49.9 \%$, the total accumulated grating strength (the $M \#$ or the sum of the induced refractive index changes of all superimposed holograms) in this material is very high, and we have measured values of the $M \# \sim 10$. This is very significant for data storage applications, as it provides a large dynamic range for superimposing holograms. 


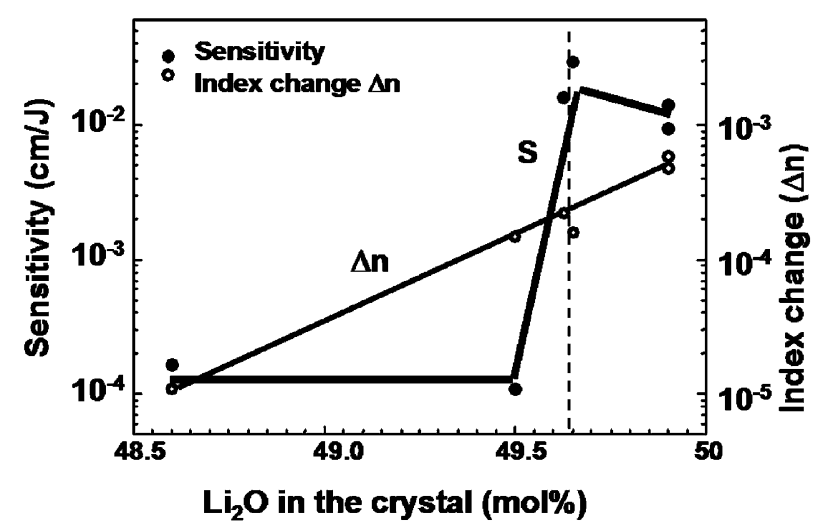

Fig. 39. Two-photon sensitivity and index change as a function of stoichiometry in undoped $\mathrm{LiNbO}_{3}$.

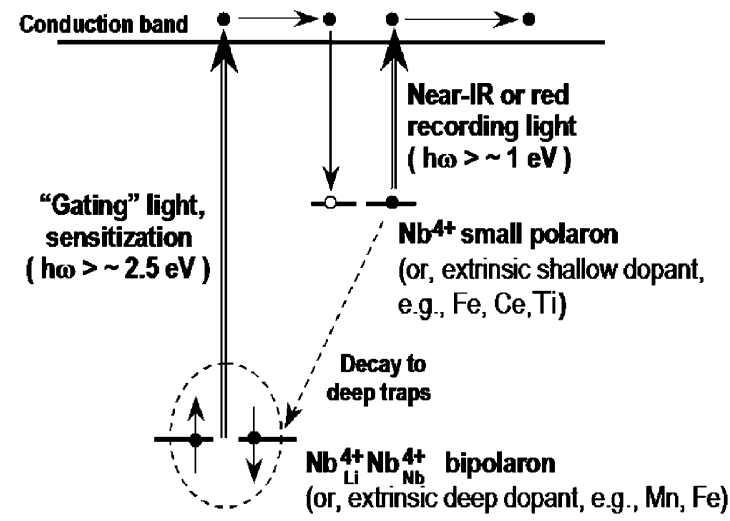

Fig. 40. Mechanism of optically gated recording in lithium niobate.

The basic physical mechanism of two-photon gated recording in $\mathrm{LiNbO}_{3}$ is shown in Fig. 40. Gating light excites electrons from deep traps (intrinsic or extrinsic) which are temporarily trapped in the shallow levels (typically small polarons or shallow extrinsic dopants). The electrons from shallow levels can be excited by red or near-IR recording light, thus forming a holographic grating. When the gating light is switched off, the electronic population in shallow levels is transferred back to the deep traps, which do not absorb the longer wavelength recording light, thus making media insensitive, allowing nondestructive readout.

The key in achieving efficient recording is in providing sufficiently long lifetimes $(>100 \mathrm{~ms}$ ) for the excited electrons in the shallow levels. This is achieved by using near-stoichiometric lightly reduced lithium niobate with low concentration of deep traps [83], [84]. Other approaches [88], [89] rely of extrinsic dopants (e.g., an Fe, Mn pair) of optimum concentration to provide pairs of deep and shallow levels within the forbidden gap of lithium niobate, similar to intrinsic defects described here.

The single photon sensitivity in the near IR is very small, thus providing a means for prolonged readout, as shown in Fig. 41. When the gating beam is turned on again during readout, information is erased.

The Stanford team [90] has implemented a digital recording system using two-photon illumination, as shown in Fig. 42. A 1-W Ti : sapphire laser pumped by a multiline

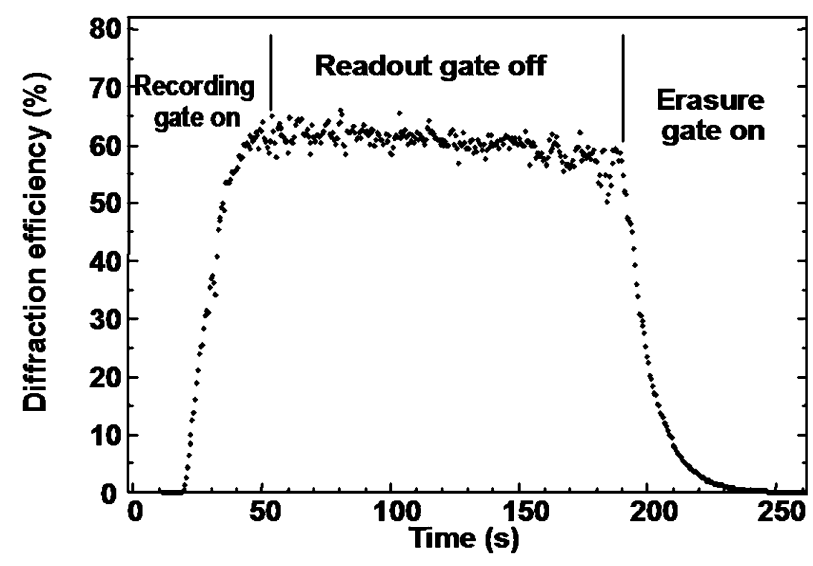

Fig. 41. Two-photon gated recording, nondestructive readout, and erasure by gating beam in near stoichiometric lithium niobate.

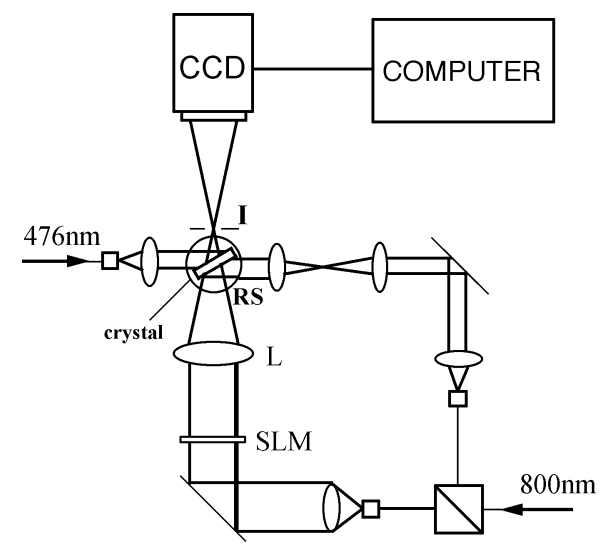

Fig. 42. Schematic of the two-photon digital holographic storage system. I: iris; L: imaging lens; RS: rotation stage.

argon laser was used to illuminate a chrome-on-glass mask containing digital data. Gating light from another argon laser at $476 \mathrm{~nm}$ is delivered to the crystal by an optical fiber. The mask pattern contained $512 \times 512,18 \times 18 \mu \mathrm{m}$ square random data bits (100\% fill factor) and alignment crosshairs, but did not include any modulation codes or ECCs. An off-the-shelf multielement lens optimized for one-to-one imaging mapped the mask pixels onto the CCD pixels. The $18 \times 18 \mu \mathrm{m}$ SLM pixels are centered on single $9 \times 9 \mu \mathrm{m}$ square CCD pixels to reduce interpixel crosstalk and relax misalignment tolerances. The surrounding nonactive CCD pixels act as a guard area that is equivalent to a smaller fill factor, and no oversampling is used for postprocessing. The crosshairs were used during the manual alignment for accurate adjustment of tilt and magnification. Closed-loop computer control of the CCD camera $x-y$ position provided fine alignment $(\sim 1 \mathrm{~mm})$ of the crosshairs before readout.

A two-photon sensitive 1-mm-thick partially reduced $\mathrm{LiNbO}_{3}$ crystal of near-stoichiometric $\left(\left[\mathrm{Li}_{2} \mathrm{O}\right]=\right.$ $49.65 \mathrm{~mol} \%$ ) composition [83] (doped with nonphotoactive $0.2 \% \mathrm{Pr}$ ) was used in these experiments. The digital holograms of the mask were recorded at different wavelengths in the transmission geometry by use of extraordinarily polarized recording beams from a tunable laser and 100 $\mathrm{mW} / \mathrm{cm}^{2}$ of gating light (Fig. 42). The imperfect parallelism of the cut crystal caused distortions of the order of one 


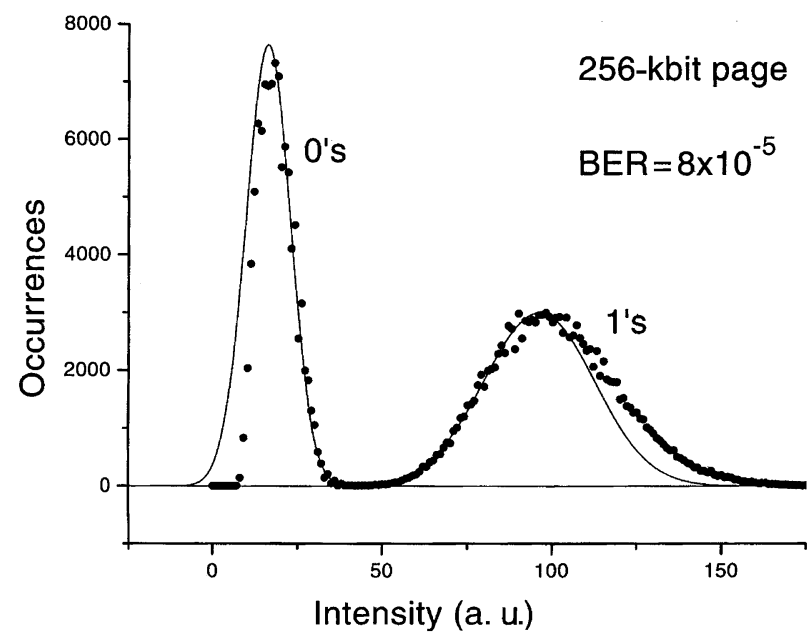

Fig. 43. Intensity distribution of data bits contained in a $256-\mathrm{kb}$ hologram after the intensities are normalized to neighboring crosshairs. The high-intensity tail of the zeros and the low-intensity tail of the ones are fitted to Gaussian functions. Their overlap is then calculated to yield a best-threshold BER estimate.

CCD pixel across the page, which were compensated for by automated realignment of the camera on the crosshairs while the hologram was scanned upon readout. For a stored 256-kb hologram, the estimated BER is found to be $8 \times 10^{-5}$ (Fig. 43), which is sufficient to guarantee a user BER of $10^{-12}$ when established modulation codes and ECCs are implemented.

\section{Other Techniques for Nondestructive Readout}

Several research results suggest that fixed hologram gratings may also be achieved using crystals with two species to establish and reveal a complementary grating with a lifetime longer than an initial principal grating; once a long-lived complementary grating is established, shorter lived gratings in a second level may be exposed and erased without significant erasure of the complementary grating. This technique has been used in $\mathrm{Bi}_{12} \mathrm{TiO}_{20}$ for real-time double-exposure interferometry [91] and image subtraction [92]. For significantly long exposure, however, the complementary grating will decay, but can have a lifetime several orders of magnitude longer than the principal grating [93], [94]. Similar applications have been demonstrated for semipermanent photochromic gratings [95], [96]. $\mathrm{KTa}_{(1-x)} \mathrm{Nb}_{x} \mathrm{O}_{3}$ [97] has been shown to display fixed holograms for gratings established in the centrosymmetric paraelectric phase at an elevated temperature and then cooled through the phase transition to a ferroelectric phase. These may be due to the participation of two photoactive species during grating formation and the deactivation of one following the phase transition.

Another mechanism to establish fixed hologram gratings related to complementary gratings relies on properties of phase transitions in ferroelectrics. Replication of photorefractive space-charge density is achieved through spontaneous polarization modulation in strontium barium niobate (SBN : 75) [98] and barium titanate $\left(\mathrm{BaTiO}_{3}\right)$ [99]. Thus, the hologram grating exists in the variations in the local spontaneous polarization of a noncentrosymmetic crystal in

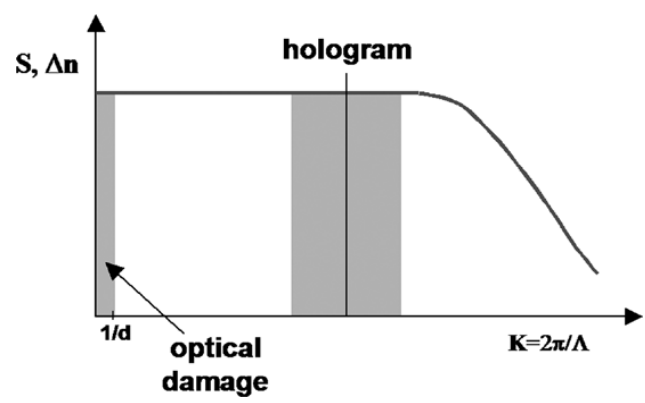

Fig. 44. Transfer function of media with a local response.

the form of microdomains whose parameters (density and average length) are correlated with the original electronic space charge [100]. This approach is effective mostly for the materials whose ferroelectric Curie temperature is close to room temperature (e.g., $\mathrm{Ba}_{0.25} \mathrm{Sr}_{0.75} \mathrm{Nb}_{2} \mathrm{O}_{6}, \mathrm{BaTiO}_{3}$ ). Using this technique, 1000 image bearing (but not digital) holograms were angularly multiplexed and fixed in Ce-doped SBN : 75 [101].

Another wavelength-dependent nondestructive readout technique relies on the spectral response of a photorefractive medium. Simple plane wave holograms written at one wavelength may be read by Bragg-matched light of another wavelength, and if the medium is not photoactive at the reading wavelength, repeated nondestructive readout is possible. For image bearing holograms, however, light at the readout wavelength cannot in general be Bragg matched to all the components of the signal. Reconstruction with spherical wavefronts has shown improved results, and strategies to determine optimal reconstruction of wavefronts have been developed [102]-[105].

In addition to fixing techniques which rely on the properties of materials, there are feedback techniques that refresh and sustain holograms during their use. Using one technique, holograms are periodically copied when their diffraction efficiency falls below a certain level [106]. Holograms may also be circulated between two photorefractive crystals by transferring data from one to the other before the holograms in the first are erased during readout, and then reversing the roles of the crystals [107]. Phased-locked sustainment may be achieved by generating a conjugate of the signal and reference beams during readout, thereby refreshing the hologram as it is read [108], [109]. By another technique, phase-locked, binarized holograms may be dynamically written, read out, updated, and refreshed using either an optoelectronic or an all-optical feedback loop for spatial thresholding in conjunction with a photorefractive crystal, as has been demonstrated in $\mathrm{LiNbO}_{3}$ [110] and $\mathrm{BaTiO}_{3}$ [111]. Finally, extended readout may be achieved by illuminating a storage material with a low-intensity reference beam during readout and enhancing the output signal using an optical amplifier [112].

\section{Noise in Photorefractive and Other Media With a Local Response}

Although $\mathrm{LiNbO}_{3}$ and other photorefractive media exhibit very low noise characteristics, as shown in the comparison 

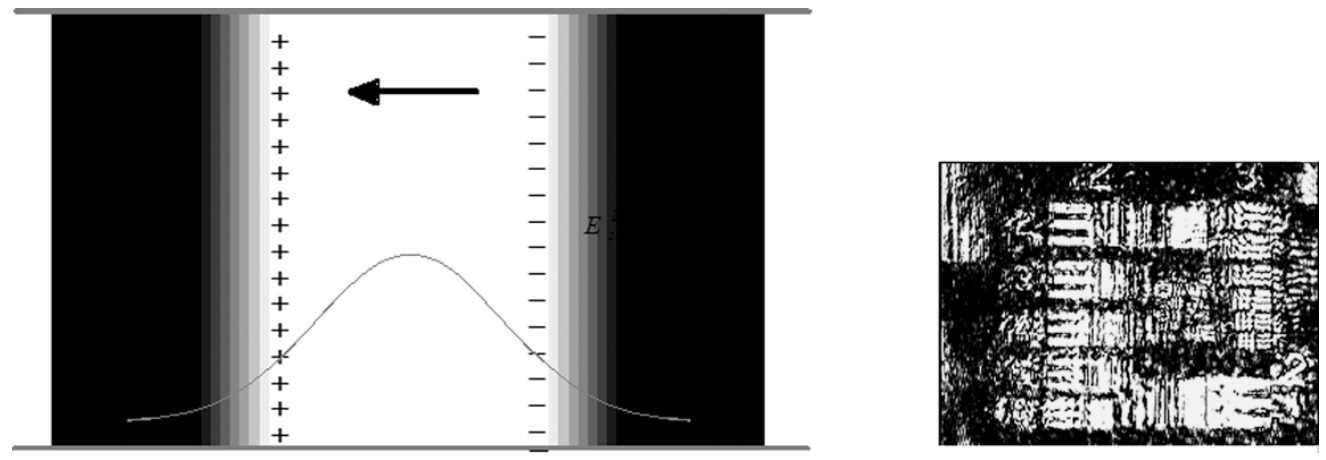

Fig. 45. The photovoltaic effect in single-crystal $\mathrm{LiNbO}_{3}$ produces large charge buildup resulting in damage that severely distorts stored data.
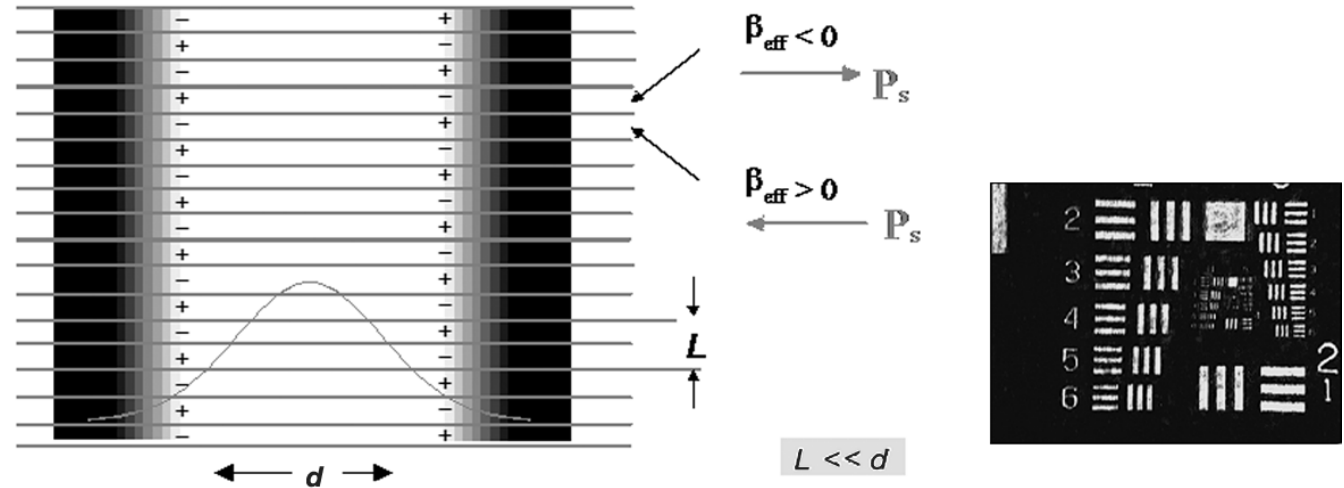

Fig. 46. Periodic poling of $\mathrm{LiNbO}_{3}$ significantly reduces photovoltaic damage.

table in Fig. 31, in the absence of significant light exposure, noise builds up due to the dc sensitivity in media exhibiting a local response, as well as photographic film and photopolymers. Here we discuss the case for $\mathrm{LiNbO}_{3}$ as it has been well studied. In $\mathrm{LiNbO}_{3}$, for example, as more and more holograms are recorded in the same location, a migration of charges in the direction of the photovoltaic current builds up local charge concentrations that lead to local index distortions in the medium. Aberrations in the readout and the data beams then result in bit errors. After a few hundred holograms are superimposed, this dc-noise effect can become significant and limits the further addition of holograms to the same location, thereby limiting the storage density. This effect is frequently referred to as optical or photovoltaic damage. Graphically the optical noise properties are shown in Fig. 44, where the sensitivity and index change are plotted as a function of the grating vector, i.e., the transfer function.

In digital holographic storage, the usable dynamic range of $\mathrm{Fe}: \mathrm{LiNbO}_{3}$ as limited by optical damage is $(M \#)_{\text {usable }}=$ 0.5-2.0 (Siros Technologies, 1996). The solution to reducing this so-called photovoltaic damage is to reduce the dc response of the medium. One effective method in $\mathrm{LiNbO}_{3}$ is periodic poling of the ferroelectric domains. For example, in bulk $\mathrm{LiNbO}_{3}$ charges build up at the edges of the crystal when illuminated by a Gaussian beam, as shown in Fig. 45, and this gives rise to distortions of the Air Force test target that is stored in the crystal (in this case a strong hologram is recorded, which gives rise to similar effects as when superimposing many weak holograms).
By alternating the direction of layers of ferroelectric domains, the dc damage effect is significantly reduced, as shown in Fig. 46. Effectively, the transfer function has a small value for low spatial frequencies, as the oppositely oriented domains prevent strong buildup of net charges [113].

\section{PhOtopolymer MATERIALS}

Early photopolymers intended for holographic applications were developed primarily for display of single images and were tolerant to a wide range of aberrations. For data storage applications, however, more stringent requirements are sought. Sensitivity, hologram shrinkage, and material stability are several of the most critical issues addressed in HDSSs based on photopolymer media. Shrinkage is particularly important because it distorts the Bragg gratings that record data holographically. Because most photopolymer processes are not reversible, photopolymer systems are best suited for WORM applications.

In general, photopolymer storage media are complex systems comprising several components and typically include the following: monomers and/or oligomers, a photoinitiation subsystem, and a chemically inactive component. The photoinitiation subsystem may further comprise a photosensitizer that is specifically chosen to sensitize the polymer to a specific light spectrum. Upon exposure to light, the photoinitiation subsystem receives photons and triggers a reaction that converts the monomers and/or oligomers to poly- 

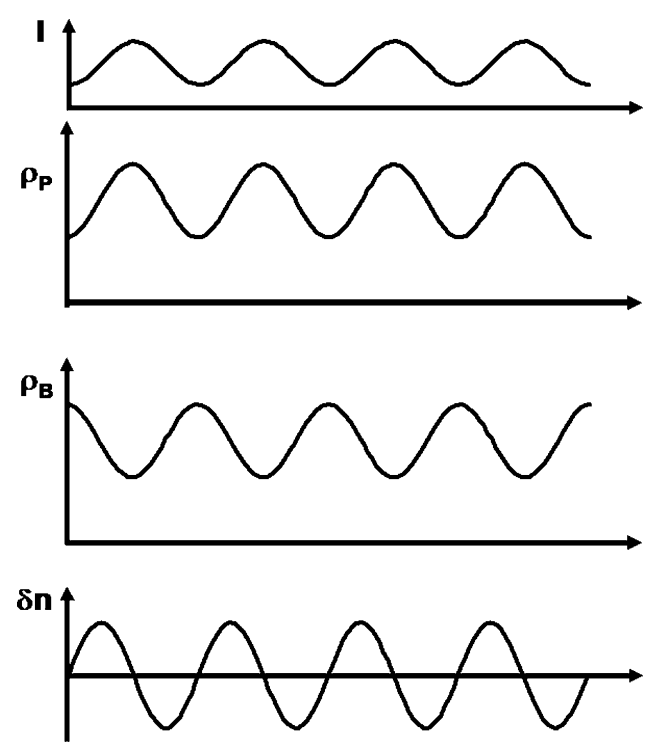

Fig. 47. Graphical depiction of the evolution of the constituent photopolymer gratings, illustrating polymer density grating $\rho_{P}$, binder density grating $\rho_{B}$, and the net index of refraction $\delta n$.

mers. Polymerization generally results in local changes in the physical density, and the chemically inactive component, frequently called a binder, may diffuse in response to the changes in density. Frequently, the binder has specific optical properties that play a role in generating the index perturbation responsible for the hologram; for example, the binder may have a different index of refraction than the monomer. Photopolymer storage media may also comprise an internal polymer scaffold that serves to enhance the stability and reduce the shrinkage of a material [16].

We now focus on a specific example of a photopolymer to illustrate the basic requirements for holographic data storage. Polaroid has developed a photopolymer system, ULSH-500, that has particularly good shrinkage properties [15]. This system uses specific monomers that polymerize using a cationic ring-opening mechanism. The photoinitiation subsystem comprises a photosensitizer and an acid generator. The photosensitizer receives light used to record a hologram and transfers energy to an acid generator. The resulting proton triggers the polymerization process, which proceeds as a monomer reacts with the acid to open a molecular ring and bond to a neighboring polymer, generating an additional proton. This reaction proceeds until it reaches a termination impurity. A particular advantage of this photopolymer system is that opening a monomer ring partially compensates for shrinkage that typically occurs during the polymerization process.

During hologram recording, monomers are polymerized in the regions of high intensity, resulting in monomer gradients between the regions of high intensity and low intensity. This is illustrated in Fig. 47, which shows the intensity distribution $I$ and the resulting polymer density grating $\rho_{P}$. Monomers then diffuse into the regions of high intensity, providing additional media for polymerization, and binder diffuses into the regions of low intensity, resulting in a binder density grating $\rho_{B}$. The resulting polymer matrix displays

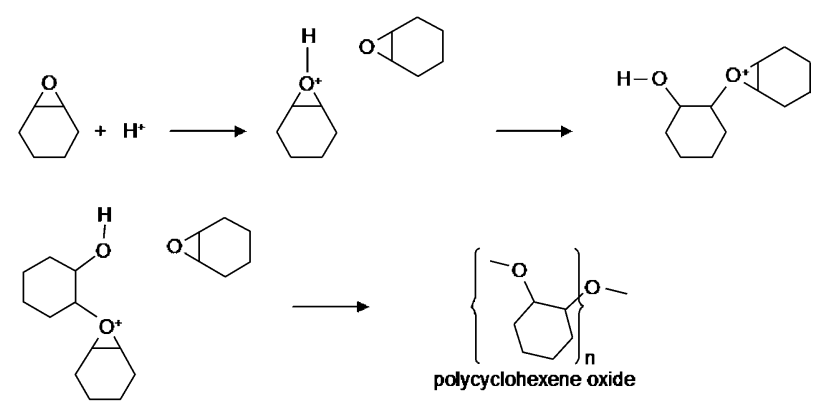

Fig. 48. Generic mechanism of polymerization and reaction chain propagation in Polaroid CROP photopolymer.

a gradient in the index of refraction due to three factors: a change in the electronic structure of the monomer upon polymerization, called molar refraction changes; density changes resulting from monomer diffusion into the regions of high intensity; and direct refractive index changes that result due to partial segregation of monomer and binder having significantly different indices of refraction. Once an index grating is established, any remaining monomer is polymerized to ensure that monomer and binder remain segregated.

Implementation of ULSH-500 in an HDSS is generally accomplished using the following procedure. Photopolymer media are deposited between two flat surfaces, typically two glass substrates, which are separated by spacers. Photopolymer thickness is generally about $100-500 \mu \mathrm{m}$; higher thicknesses can be achieved but generally require a decrease in sensitizer concentration, and, therefore, recording speed, to maintain usable optical density. Prior to polymerization, the medium is a medium viscosity fluid. An initial preexposure process, therefore, is typically required to establish a photopolymer matrix, or scaffold, to provide the necessary material stability prior to recording. The preexposure process may be accomplished by thermal treatment and/or flood exposure to light. After preexposure the material is converted into a gel-like state in which holograms can be recorded. After the final hologram is recorded, a postexposure process may be applied to polymerize any remaining monomer, thereby improving the stability of the material. Single hologram diffraction efficiencies can approach $100 \%$; as with inorganic materials, significantly less diffraction efficiency per hologram is obtained for multiplexed holograms, as the dynamic range is budgeted among the recorded holograms. Recording sensitivities can be about $3 \mathrm{~cm} / \mathrm{mJ}$ for light at wavelength of $514.5 \mathrm{~nm}$, and exposure fluencies of about 50 $\mathrm{mJ} / \mathrm{cm} 2$ can result in single-hologram diffraction efficiencies of about $80 \%$ for $100 \mu \mathrm{m}$ thick photopolymer media. The high sensitivity of CROP photopolymer is merely because of the chain propagation effect (as shown in Figs. 48 and 49) as well as the relatively high molecular weight of the stating monomers [15].

A critical problem related to photopolymer media is shrinkage. As the medium is exposed to light, it changes from a gel-like substance to a solid plastic. As a result, the density of the medium increases as the volume shrinks. As a consequence, the grating spacing of previously recorded data changes as more holograms are superimposed. This 


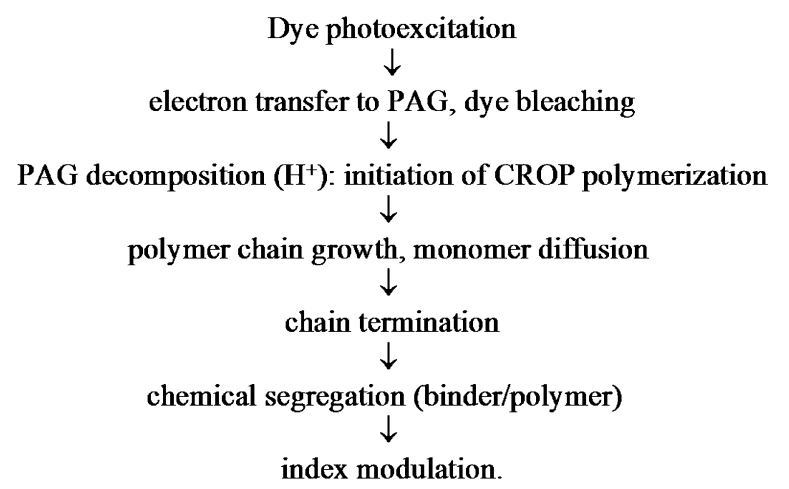

Fig. 49. Mechanism of hologram recording in Polaroid CROP. PAG stands for photo acid generator.

leads to Bragg mismatch, as the grating wave vector is increased due to shrinkage. As a result, the readout beam must be altered in order to satisfy the Bragg condition for efficient readout. In practice, either the readout wavelength or the readout beam angle is changed compared with the reference beam used for recording; usually both are changed [114]. For reconstruction of a plane wave, this can often be accomplished, however, for data bearing object beams, all grating vectors usually cannot be simultaneously Bragg matched, and as a result, only a portion of the data page is read out for a particular readout beam geometry. This, of course, leads to potentially large data errors and must be avoided. In practice, this can be achieved by optimizing the system for a certain amount of tolerable shrinkage in the medium. By trading off recording sensitivity by preexposing the medium so it becomes closer to its final solid state, shrinkage is reduced, but the available dynamic range is reduced commensurately as well, and fewer holograms can be recorded with lower sensitivity, requiring either longer exposure times or higher recording powers. As CROP materials exhibit lower shrinkage per unit volume for a given sensitivity than free-radical media, they have practically proven to be the best performing media for holographic data storage. Their optical properties are optimized for a particular holographic storage system by varying composition of the constitutive photopolymer components. Overall system performance is optimized by carefully considering all system and media characteristics and determining the optimum capacity at a certain data transfer rate given limitation on cost and system components.

A HDSS based on photopolymers is typically implemented using a rotating disk, as this provides a convenient format for using optomechanical systems developed for other types of optical storage. Multiplexing techniques best implemented in a disk-based architecture include rotational multiplexing (similar to shift multiplexing), which is based on a reference beam with curved wavefronts [11], and speckle multiplexing, which is based on a reference beam with a speckle pattern. Rotating the medium decorrelates the reference beam with respect to the recorded hologram, thus enabling multiplexing by simply rotating the disk. This type of photopolymer system has been implemented in HDSSs described in more detail below.
A variety of other photopolymer systems are under development as candidate materials for HDSSs. Lucent, for example, has developed a free-radical photopolymer system that utilizes two independently polymerizable and compatible chemical subsystems [115]. One subsystem defines a polymer matrix scaffold in situ using low-index polymer precursors. The other subsystem records holograms using highindex monomers, which remain intact after polymerization of the first subsystem. The polymer scaffold enhances the stability of the material and allows it to be shaped in a variety of thick and flat formats. The scaffold is further designed to avoid cross reactions with the monomer subsystem that would reduce the refractive index contrast. The Lucent team has reported implementing this type of photopolymer system in a shift-multiplexing architecture to record about 4000 holograms in a configuration having a potential of about $1 \mathrm{~Gb}$ of data, which is equivalent to about 50 Gbytes of user capacity in a 5.25-in disk [116]. These authors do not report any BER values, making evaluation of the storage capacity of these experiments somewhat difficult.

\section{OPTICAL SySTEM ARCHITECTURE ISSUES}

Design of HDSSs has been studied by many authors, from the early 1970s till recently. In early work [117], Vander Lugt, for example, examined the effects of optical constraints on the information storage capacity of block oriented pagebased memory systems. He did not take multiplexing of data into consideration in order to keep the SNR high and showed that the packing density is limited by geometrical constraints of the Fourier and imaging optics. The areal information density $D$ (raw) for holographic storage can be approximated as a product of the individual hologram density (calculated in [117] and [118]) times the number of superimposed holograms $N$

$$
D=\left(\frac{d}{\zeta \lambda F}\right)^{2} \times N
$$

where $d$ is the size of the SLM, $\lambda$ is the wavelength of light, $F$ is the focal length of the imaging optics, and $\zeta$ is a dimensionless parameter which relates the actual hologram size to the Nyquist limit (typical values used in holographic storage systems are within the range from 1.1 to 1.6 , depending on the image quality requirements). The number of holograms which can be superimposed is limited by a variety of parameters such as required transfer rate (i.e., photon budget), material dynamic range (i.e., $M \#$ ), scatter, and information crosstalk. In order to achieve high storage density, it is, therefore, important to achieve high data density per hologram, which according to (51) requires a high NA for the imaging optics. The tradeoff between capacity and transfer rates is estimated by computing the number of photons incident on a detector element during page readout. From this calculation, we can estimate the maximum number of superimposed pages readable with a certain SNR

Number of pages $=\left(\frac{2 M \#^{2} \cdot q \cdot \mathrm{QE} \cdot P \eta_{\mathrm{opt}} \eta_{\text {fix }} t_{\text {read }}}{P_{x} P_{y} p_{\min }}\right)^{0.5}$ 


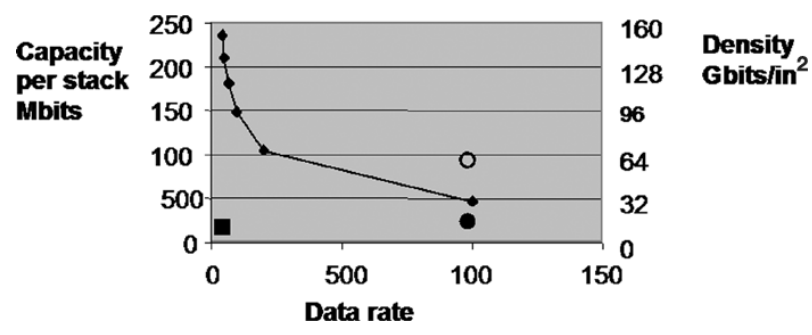

Fig. 50. Tradeoff between capacity and transfer rate for the parameters described in the text. The square dot denotes MO recording at $2 \mathrm{~Gb} / \mathrm{in}^{2}$ at $40 \mathrm{Mb} / \mathrm{s}$. The round filled dot (just above the "1000" marker) represents the Stanford/Siros Gb/s demo, while the circle (above the endpoint of the solid line) denotes the planned final HDSS demonstration performance.

where $M \#$ is a variable based on system and materials parameters, $q$ is the number of photons per watt per second, $\mathrm{QE}$ is the quantum efficiency of the detector array, $\eta_{o p t}$ is the optical efficiency of the readout system, $\eta_{\text {fix }}$ is the fixing efficiency, $t_{\text {read }}$ is the read time of the recalled data, $P_{x} x P_{y}$ is the number of pixels in the data page, $p_{\min }$ denotes the number of photoelectrons needed to get a $20-\mathrm{dB}$ SNR, and $P$ is the power of the readout beam. It is assumed that bit patterns are balanced (although this does not have to be the case) by making half the pixels in a page on and half off. From this equation, the tradeoff between capacity (the product of the stored number of pages, the code rate, and the number of bits per page) and data transfer rate (the number of bits per page times the code rate divided by the readout time) is determined in a photon-limited system. Optical damage and crosstalk limitations are not considered. In practice, however, optical damage often tends to limit capacity, but can be significantly reduced by recording at elevated temperatures [68] or by using periodically poled media. Equation (52) provides an upper bound.

As an example, assume that $p_{\min }=10000$ photoelectrons to achieve a $20-\mathrm{dB}$ SNR, the page size is $P_{x} x P_{y}=$ $1024 \times 1024$, the number of photons per watt per second is $q=3 \times 10^{18}$, the dynamic range is $M \#=1$, the camera frame rate is $1000 \mathrm{ft} / \mathrm{s}$, i.e., $t_{\text {read }}=1 \mathrm{~ms}$, the detector quantum efficiency $=0.3 @ 0.7 \mu \mathrm{m}$, the laser power $P=5 \mathrm{~W}$, the fixing efficiency $\eta_{\mathrm{fix}}=0.5$, and the optical readout efficiency $\eta_{\text {opt }}=0.5$; then the number of pages per stack is approximately 470 , and the number of stacks for $100-$ GB raw capacity is 2100 . Thus, high data capacities and transfer rates require substantial spatial multiplexing.

However, capacities of several gigabytes could be achieved with no spatial multiplexing for data transfer rates near a few megabytes per second. Using acoustooptic Bragg cells, access times can be made less than $50 \mathrm{~ms}$ with no moving parts. At a transfer rate of $5 \mathrm{MB} / \mathrm{s}$ - typical for current magnetooptic drives - data density for HDSS is 50-70 times higher using a laser with two orders of magnitude more power. By varying the materials and systems parameters according to (52), the curve of Fig. 50 can be modified. In particular, improvements in $M \#$ are effective in increasing storage capacity, an area of much current research. Further improvement is achieved by using signal processing and coding techniques.

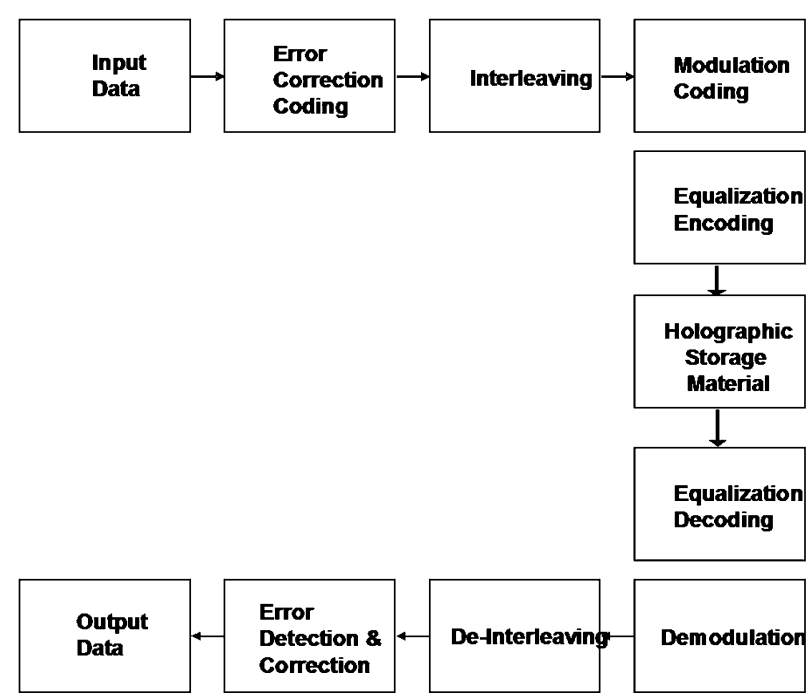

Fig. 51. Typical signal path in an HDSS.

\section{Signal PROCessing AND EN/Decoding}

In a data storage system, the goal of error correction coding, data modulation, and signal processing is to reduce the BER to a sufficiently low level while achieving such important figures of merit as high density and high data rate. The distinct feature of digital holographic data storage is that this technology treats each individual pixel of the hologram as a data channel and the entire image as set of data channels. This allows the employment of modulation coding and signal processing techniques to improve the system performance in terms of total capacity at an acceptable error rate.

Coding and signal processing can involve several qualitatively distinct elements. The cycle of user data from input to output can include error correction coding, interleaving, modulation encoding, signal preprocessing, data storage in the holographic system, hologram retrieval, analog signal detection and binary digitization (using CCD or CMOS detector array), signal postprocessing, channel decoding, and ECC decoding of deinterleaved binary data.

A simple example of a signal path in an HDSS is shown in Fig. 51. Input data are processed to generate data blocks with error correction coding. The data are interleaved among blocks to distribute burst errors among multiple blocks. Data are subject to a modulation code that converts binary data into specific modulation formats to be recorded in a holographic material. On readout, data are demodulated and then deinterleaved. The error correction algorithm is applied to correct and/or identify error, generating the output data.

The purpose of modulation coding is to simplify the detection of the analog signal values from the detector array. Modulation codes generally restrict available patterns for formatting data to a subset that are less likely to be corrupted during recording, storage, and/or readout. This restriction is typically achieved using constraints on the types of data patterns that may occur. A balanced constraint addresses the issue of variation in signal strength across a data page; in a system with binary channels, this issue is addressed by ensuring that 
a fixed number of OFF and ON pixels (zero and one, respectively) occur in a block of a given size. A low pass constraint addresses intersymbol interference arising in high-frequency data patterns, typically by eliminating the data patterns most susceptible to intersymbol interference.

The simplest detection scheme is global threshold detection, in which a specific threshold value is chosen: any CCD pixel with an intensity above the threshold is declared a one, while the rest are declared zero.

If all holograms result in equal diffraction efficiencies, a threshold intensity can be chosen to distinguish ON pixels from OFF pixels. Note that we use OFF and ON when referring to SLM or CCD pixels and "zero" and "one" when referring to information bits.

For example, the probability of an error given that a zero is transmitted in a threshold detection system is given by [119], [120] assuming a model of additive white noise which is independent from pixel to pixel

$$
p \approx \int_{a_{T}}^{\infty} \frac{1}{\sqrt{2 \pi} \sigma} \exp \left(-\frac{\left(r-a_{L}\right)^{2}}{2 \sigma^{2}}\right) d r
$$

where and $a_{L}$ is the average amplitude of OFF pixels at the $\mathrm{CCD}$ array, $\sigma$ is the standard deviation of the noise $a_{T}$, is the threshold value set to be the mean of the ON and OFF values, or, in more general case of different statistics for ones and zeros, set to optimal value which minimizes the total BER. The latter is achieved when the threshold $a_{T}$ is set to the point where the ones and zeros' intensity distributions cross each other.

In the presence of variation in signal strength across a data page, this simple scheme does not perform satisfactorily because the optimal value of the threshold becomes dependent on the position within the page. A modulation code identified as an early alternative to the threshold approach in HDSSs is a balanced constraint known as differential decoding in which each zero and one of the data are encoded in form of a group two adjacent pixels a and b, one ON, one OFF [24]. Upon detection and demodulation, if the intensity of pixel a is lowest, the data bit is declared a zero, and if the intensity of pixel b is lowest, the data bit is declared a one. Viewed a different way, a zero-one sequence represents a data bit zero and a one-zero sequence represents a data bit one.

The probability of an error in a differential detection system with additive white noise is given by

$$
\begin{aligned}
p \approx \int_{0}^{\infty} \frac{1}{\sqrt{2 \pi} \sigma} & \exp \left(-\frac{\left(r-a_{L}\right)^{2}}{2 \sigma^{2}}\right) \\
& \times \int_{0}^{r} \frac{1}{\sqrt{2 \pi} \sigma} \exp \left(-\frac{\left(x-a_{H}\right)^{2}}{2 \sigma^{2}}\right) d x d r
\end{aligned}
$$

where $a_{H}$ represents the average amplitue of the ON pixels.

Most notably, the error rate is independent of the choice of the threshold value, thus making differential encoding very robust and very easy to implement. This technique largely solves the problems associated with nonuniformity of holographic data pages. Significant improvements in raw BER have been made using differential encoding over simple thresholding schemes [121]. Another advantage to the differential encoding technique is that it can be used when single pages reconstruct nonuniformly. This nonuniformity can result from poor overlap of signal and reference beams in the crystal, from nonuniform illumination of the SLM, or from the introduction of interference fringes due to multiple reflections in the optical system. An important disadvantage of this approach is a relatively high coding overhead due to the constraint to use two pixels to encode a single data bit.

The code rate can be improved by increasing the array size and employing mathematically more sophisticated coding and decoding strategies [121]. More advanced balanced modulation codes provide local thresholding capability and relatively high code rates (75\% and higher). These codes include balanced block codes [122] in which the fixed size pixel codeword, or data block, can contain only a specified number of ON pixels. An example is the $6: 8$ code, in which a data pages is divided into $2 \times 4$ arrays, each containing exactly four ON pixels and four OFF pixels. The number of permutations for this constraint is 70 , which can support $6 \mathrm{~b}$.

Other modulation codes include parity threshold codes in which the number of ON and OFF pixels in a large uncoded array is stored in a small coded (with highly redundant code) subarray, strip codes [122], and soft-decision iterative turbocodes. In the case of block codes, the detection is based on a sorting or correlation technique, in which the detected block of pixels is compared to the set of valid code words and is declared to be a specific code word based on maximum likelihood criteria. Most advanced channel codes with large coded array sizes also provide intrinsic error correction capability due to highly redundant coding, which allows correction of a limited number of pixels directly during the channel decoding step. An example of a code with a large coded array size is the $8: 12$ strip balanced array code [123].

Intersymbol interference can be introduced by a number of factors, such as the optical bandpass of an HDSS, misregistration of the imaged signal page and detector array, crosstalk between pixels in an SLM, and electrical crosstalk between detected signal pixels. Optical misregistration can be addressed using sophisticated interpolation algorithms based on remapping the received data pages [124] or serial correction processing techniques [125]. Other techniques for reducing the negative effects of intersymbol interference in page detection include Vitterbi algorithms [126], image deconvolution upon detection [127], and equalization via predistortion and inverse filtering during the recording [128].

Another approach is to use passband codes that eliminate certain data patterns responsible for the highest incidence of data error due to intersymbol interference, such an OFF pixel surrounded by all ON nearest neighbors [122].

The ECC adds redundancy to the data in order to provide protection from various noise sources. The ECC-encoded data are then interleaved and passed on to a modulation encoder, then input to the SLM and stored in the recording medium. On the retrieving side, the CCD returns pseudoanalog digitized data values, which then undergo a 


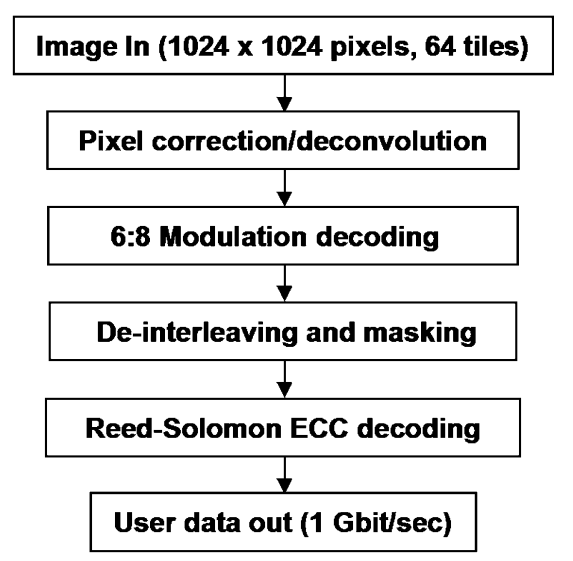

Fig. 52. HDSS electronics operation.

postprocessing step (e.g., deconvolution in order to compensate for the smearing of the optical signals due to image distortion and diffraction point spread function). The array of the processed digital data is further passed to the modulation decoder, which performs the inverse operation to modulation encoding, then to the deinterleaver, and, finally, to the ECC decoder. The purpose of data interleaving is in reduction of the corrected BER (i.e., after the ECC encoding) by making the digital data error rate before ECC encoding uniform. Even with balanced modulation code, the BER may not be uniform across the page. Thus, by corrupting different parts of the same ECC block in different locations in the page, uniform BER distribution between different ECC blocks can be achieved, thereby minimizing the total BER after the ECC decoding.

As research in HDSSs progresses, more sophisticated approaches, including algorithms implemented in one or a few chip sets, can be incorporated based on advances in data storage and communications industries.

\section{ELECTRONIC IMPLEMENTATION}

The purpose of the specialized processing electronics is to provide the holographic channel decoding, deinterleaving, ECC decoding, and overall system synchronization, all at high data rates $(1 \mathrm{~Gb} / \mathrm{s})$. This cannot be accomplished (with existing computers) in software due to both processing speed and timing limitations. The functionality of channel electronics is best described on the basis of the first ever hardware implemented system developed by Siros Technologies and Stanford for HDSS consortium.

The HDSS electronics are based on field-programmable gate array (FPGA) technology and can be reprogrammed using software and hardware means. HDSS channel-decoding electronics consists of three main processing blocks: holographic channel decoders, a demultiplexer, and a Reed-Solomon ECC board. Basic processing steps of the electronics are shown in Fig. 52.

Pixel deconvolution is performed on the raw 8-b pixel values. The $3 \times 3$ kernels are computed in software and provide pixel corrections to reduce the effect of optical point-spread function and distortions of the imaging system, thus increasing the SNR of the input image.

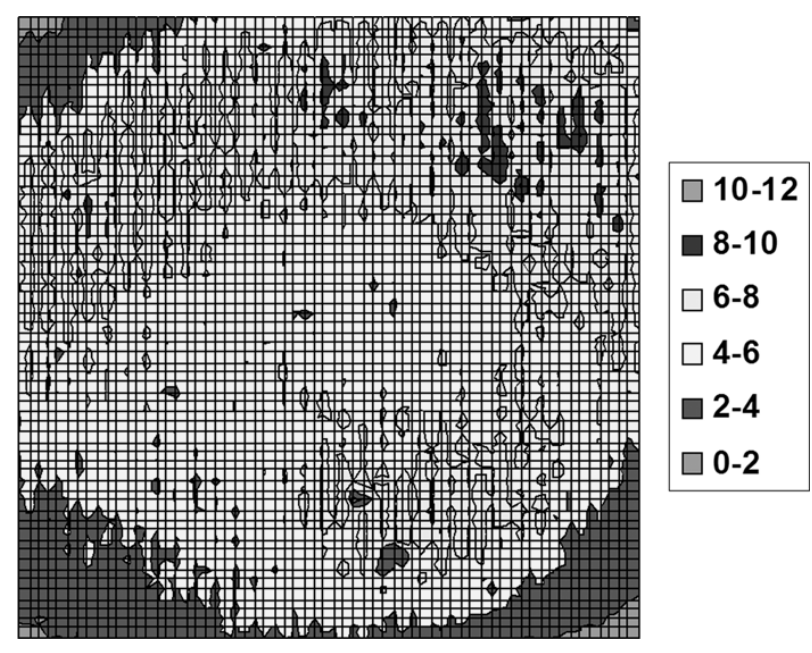

Fig. 53. Image SNR distribution.

The decoder boards provide $6: 8$ channel decoding. A simple $6: 8$ code (i.e., 8 pixels correspond to $6 \mathrm{~b}$ of data, including ECC bits) was chosen for the HDSS holographic disk system because it provides a fairly high (75\%) code rate and has sufficiently low complexity to be implementable in hardware. More advanced codes (e.g., 8:12 strip code) provide somewhat better performance [123] with respect to BER and storage capacity, but are significantly more complex for hardware implementation.

The purpose of data interleaving is in reduction of the corrected (i.e., after ECC) BER. The SNR of the source images is usually not uniform across the different image sections. By distributing data in different sections of the image and deinterleaving the decoded data, one can achieve uniform BERs in all the ECC sequences before error correction operation, thus reducing the error rate of the user data. Areas of high BER (SNR $<\sim 2.0$ ) are usually excluded (masked) from the page. The masking operation is also performed by the demultiplexer electronics. The deinterleaving and masking look-up tables are generated by software, based on the hologram SNR distribution (such as the one shown in Fig. 53) and are downloaded to the electronics during the electronics initialization step.

During hologram recording and readout, the synchronization electronics provide timed pulses to the SLM, the CCD camera, and the laser according to the address table, which is downloaded before the beginning of high-speed operation. The HDSS decoding electronics are triggered automatically by the CCD camera after each camera exposure.

\section{Demonstration Platforms}

A number of systems, including the first digital HDSS, were designed, built, and tested at Stanford University and Siros Technologies for a variety of purposes. They were built under sponsorship of the Center of Nonlinear Optical Materials and the PRISM and HDSS consortia. The primary goals were to gain insight into the underlying physics of the recording mechanisms and into the system tradeoffs required 


\begin{tabular}{|l|l|l|l|l|l|l|}
\hline Date & Name & Affiliation & $\begin{array}{l}\text { Purpose of } \\
\text { Demo }\end{array}$ & Capacity & Density & I/O rate \\
\hline $\begin{array}{l}\text { Mar- } \\
94\end{array}$ & $\begin{array}{l}\text { Science } \\
\text { System }\end{array}$ & $\begin{array}{l}\text { Stanford } \\
\text { University }\end{array}$ & $\begin{array}{l}\text { First fully } \\
\text { functional } \\
\text { system }\end{array}$ & 0.2 & 0.3 & 0.1 \\
\hline $\begin{array}{l}\text { Dec- } \\
95\end{array}$ & $\begin{array}{l}\text { Next step } \\
\text { TV Demo }\end{array}$ & Siros & $\begin{array}{l}\text { First compressed } \\
\text { video demo }\end{array}$ & 5 & 3.4 & 1.7 \\
\hline $\begin{array}{l}\text { Mar- } \\
96\end{array}$ & $\begin{array}{l}\text { Non- } \\
\text { volatile } \\
\text { LiNbO3 }\end{array}$ & Siros & $\begin{array}{l}\text { First long term } \\
\text { storage demo }\end{array}$ & 10 & 1.3 & 1.7 \\
\hline $\begin{array}{l}\text { May- } \\
96\end{array}$ & $\begin{array}{l}\text { High } \\
\text { speed I/O } \\
\text { demo }\end{array}$ & Siros & $\begin{array}{l}\text { First 1000 } \\
\text { frames per } \\
\text { second readout }\end{array}$ & 0.4 & 5.0 & 45 \\
\hline $\begin{array}{l}\text { Spe- } \\
96\end{array}$ & $\begin{array}{l}\text { FPGA full } \\
\text { system }\end{array}$ & Siros/GTE & $\begin{array}{l}\text { First fully } \\
\text { electronic I/O }\end{array}$ & 11 & 6.3 & 1.7 \\
\hline $\begin{array}{l}\text { Apr- } \\
99\end{array}$ & $\begin{array}{l}\text { Full HDSS } \\
\text { demo }\end{array}$ & Stanford/Siros & $\begin{array}{l}\text { l Gbit/sec } \\
\text { readout }\end{array}$ & 1.5 & 2.5 & 1000 \\
\hline $\begin{array}{l}\text { Sept- } \\
\text { 00 }\end{array}$ & $\begin{array}{l}\text { 10 } \\
\text { Gbit/sec } \\
\text { demo }\end{array}$ & Stanford & $\begin{array}{l}\text { Record 10 } \\
\text { Gbit/sec readout }\end{array}$ & $\begin{array}{l}1.5 \\
2.5\end{array}$ & 10,000 \\
\hline
\end{tabular}

Fig. 54. Stanford/Siros demonstration platforms.

to achieve specified performance, and to integrate new components into working demonstration platforms. The most significant demonstrations are tabulated in Figs. 54 and 55 and are briefly described in ensuing sections.

\section{A. Stanford University All-Digital System Demonstration (1994)}

In 1994, we demonstrated the first fully digital HDSS [24] by storing digital images and a short soundtrack. The purpose of this experiment was to show that a fully functional system could be implemented with off-the-shelf components.

Particular emphasis was placed on using digital signal processing techniques to overcome noise issues limiting capacity and transfer rates. A new differential coding scheme was introduced that allowed us to significantly increase capacity by adding extra bits for channel and ECC coding. It allowed us to evaluate the tradeoffs between storage system capacity, BER, and data transfer rate for several channel and ECC coding schemes.

The capacity is limited for the most part by the attainable diffraction efficiency of the stored holograms. As the number $N$ of holograms stored in a single stack increases, the diffraction efficiency falls as $1 / N^{2}$, mentioned above. As the strength of the diffracted signal decreases, the SNR decreases because the strength of noise due to scatter and CCD noise is independent of $N$. The total number of holograms that can be stored is, thus, determined by the minimum acceptable SNR. A HDSS is also limited by page-to-page fluctuations in signal strength, which can occur due to laser instability or, for example, due to a nonoptimal choice of the recording schedule. Intrapage distortion can occur due to imperfections in the optical system or in the SLM or CCD array. These noise sources limit the applicability of direct storage of images in analog form. The majority of previous holographic storage implementations have involved direct storage and retrieval of pictorial information. For example, Mok has demonstrated angular-multiplexed storage of as many as 5000 edge-enhanced analog images in a single crystal [8].

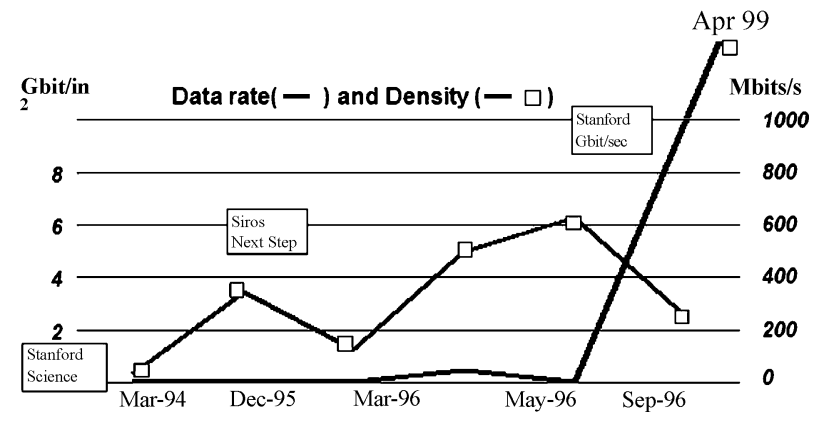

Fig. 55. Siros and Stanford University demonstration platforms in historical order. The laser power for all experiments was $400 \mathrm{~mW}$, except for the April 1996, experiment which used a 250-mW laser.

The noise-tolerant nature of digital storage makes it possible to overcome problems associated with the aforementioned noise sources. However, prior to the publication of [24], no fully automated digital HDSS had been implemented and no comprehensive study of the bit-error-rate performance of a system operating at reasonable data transfer rates had been carried out. Achievable BERs have been projected previously by sampling a small number of digital information bits from a random sampling of 1000 holograms stored in a manually Bragg-tuned system [129]. Using various encoding techniques, we have implemented what to the best of our knowledge was the first fully automated system in which data are written and recalled in digital form. We have used this spatio-rotational multiplexing system to store digital representations of color images and compressed video. Critical features of our implementation that allowed us to overcome previous obstacles include location of the Fourier plane outside the crystal volume, use of cylindrical lenses to implement spatial multiplexing, a novel differential encoding technique used to increase error immunity, use of well-known Hamming error correction codes, and the distribution of consecutive information bits over multiple data pages in order to decrease the probability of burst errors. With these techniques, BERs of $10^{-6}$ have been achieved at 


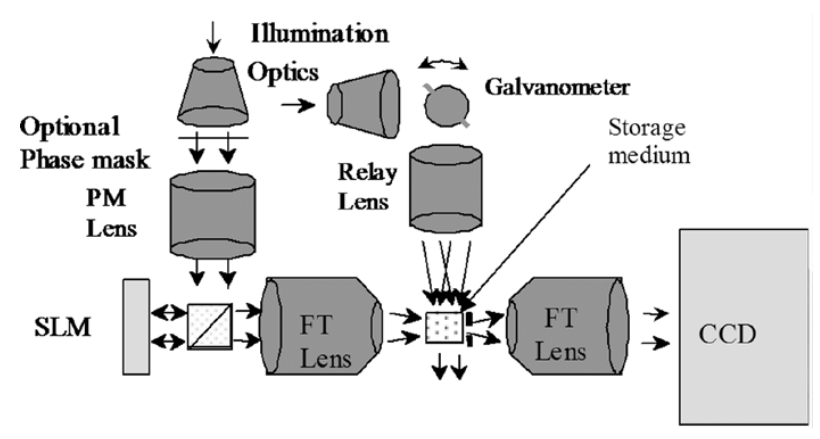

Fig. 56. Generic perpendicular $\left(90^{\circ}\right)$ recording geometry.

readout rates of $6.3 \times 10^{6}$ pixels per second. We have used the system to evaluate tradeoffs between BER and storage capacity at a fixed data transfer rate.

A generic diagram of our system is shown in Fig. 56. The storage medium is an $\mathrm{Fe}$-doped $\mathrm{LiNbO}_{3}$ crystal cut such that its $c$ axis was at $45^{\circ}$ to the crystal surfaces. The crystal dimensions are $2 \mathrm{~cm} \times 1 \mathrm{~cm} \times 1 \mathrm{~cm}$, of which only a $0.1-\mathrm{cm}^{3}$ portion was used for data storage. The crystal was mounted on a computer-controlled rotation stage, which was in turn mounted on a computer-controlled translation stage, allowing the crystal to be moved in the vertical direction. The SLM was a $480 \times 440$ pixel liquid crystal array taken from an InFocus TVT-6000 video projector. It was addressed with an analog video signal produced by a framegrabber board in the computer. The camera was an intensified CCD array. Each stored hologram was read out in 1/30 s. The cylindrical lenses in the reference beam path were used to collimate the reference to an area of approximately $10 \mathrm{~mm} \times 2 \mathrm{~mm}$ at the face of the crystal. The combination of the translation stage and the collimation of the reference beam allowed holograms to be stored in four different stacks. The signal beam occupied an area of approximately $1.5 \mathrm{~mm} \times 1.5$ $\mathrm{mm}$ on the front face of the crystal. The Fourier plane was located approximately $3 \mathrm{~mm}$ in front of the crystal. A filter was used to select only the central spot of this transform. The filter prevented erasure of previously recorded stacks during writing and eased alignment tolerances at the CCD plane. By recording in a Fresnel plane, rather than the Fourier plane, the modulation depth was sufficiently uniform to eliminate the need for a diffuser [130]. Because previously recorded holograms were erased in this demonstration as additional holograms were recorded, an appropriate recording schedule had to be determined in order to store a large number of pages with equal diffraction efficiency [2].

If all holograms result in equal diffraction efficiencies, a threshold intensity can be chosen to distinguish ON pixels from OFF pixels. Note that we use OFF and ON when referring to SLM or CCD pixels and "zero" and "one" when referring to information bits. An incorrect measurement of the time constant can result in a schedule which leads to unequal diffraction efficiencies for each of the stored holograms. A $10 \%$ error in the assumed time constant can result in more than a factor of two variation in diffraction efficiency. In addition, laser fluctuations or anomalous writing behavior can result in unequal diffraction efficiencies.
To address these issues, we used the differential encoding technique described above, in which the pixel sequence $\mathrm{OFF}-\mathrm{ON}$ represents a data bit zero and the pixel sequence $\mathrm{ON}-\mathrm{OFF}$ represents a data bit one. In addition to being insensitive to page-to-page intensity fluctuations, the differential encoding technique results in a lower probability of error assuming a model of additive (in amplitude) white noise which is independent from pixel to pixel as described above. Our experiments showed for the first time that significant improvements can be made using differential encoding over simple thresholding schemes [24]. Another advantage to the differential encoding technique is that it can be used when single pages reconstruct nonuniformly. This nonuniformity can result from poor overlap of signal and reference beams in the crystal, nonuniform illumination of the SLM, or the introduction of interference fringes due to multiple reflections in the optical system. When a substantial contribution to the variance is due to such large scale nonuniformities, we expect difference encoding to offer further improvement over simple thresholding techniques.

Ideally, each pixel on the SLM would be used to represent a single bit of data. The liquid crystal array used in these experiments exhibited some interpixel crosstalk. In other words, pixels adjacent to an ON (transmitting) pixel exhibit a significant amount of transmission. Also, the SLM and the CCD array were manufactured with different horizontal to vertical pixel pitch ratios. Thus, using a simple optical system such as ours, it was impossible to image the SLM onto the CCD array such that each SLM pixel is imaged directly onto a CCD pixel. In practice, a multiple lens arrangement could be used to achieve the proper anamorphic imaging; however, this greatly increases system complexity. The pitch mismatch resulted in a systematic geometrical source of errors if too fine a sampling grid is used. In order to circumvent these problems, we used a block of $8 \times 8$ pixels to represent $1 \mathrm{~b}$. The differential encoding technique increases the pixel-to-bit ratio to $8 \times 16$ pixels per information bit. The data is read out by sampling the CCD output at one pixel for each $8 \times 8$ pixel block imaged onto it. The difference in intensity between adjacent samples is used to determine whether the information bit is a zero or a one.

We measured the raw BER of our system to be between $10^{-3}$ and $10^{-4}$ at video readout rates. In order to improve performance, we implemented a Hamming error correcting code in which four check bits were added to each string of eight data bits. The Hamming code is capable of correcting a single bit error in the sequence of $12 \mathrm{~b}$ assuming that only one bit error occurs in those 12. In order to reduce the potential of burst errors, each stored data page represented one bit plane in a two-byte sequence. Thus, each bit in the 12-b sequences was stored in a separate page in order to reduce the probability of multiple errors within a given sequence. With the error-correcting codes, we were able to achieve BERs of $10^{6}$, which is adequate for compressed video storage and more than sufficient for uncompressed image storage. We used our system to store both digital color images and low-resolution video data. The total capacity of the system was 1232 pages with $1592 \mathrm{~b} /$ page, resulting in a raw capacity of 245 
$\mathrm{kB}$. Taking into account the necessary error-correcting bits, the total useful data capacity was $163 \mathrm{kB}$. The total pixel capacity of our system was $2.6 \times 10^{8}$ pixels, with a density of $3 \times 10^{9}$ pixels per $\mathrm{cm}^{3}$. The transfer rate is $6.3 \times 10^{6}$ pixels per second. The CCD camera outputs $1 \mathrm{~B}$ per pixel. The total pixel capacity of our system indicates that our storage capacity does not represent a fundamental limit. Rather, the primary limitation in determining the information storage capacity is the necessary oversampling on the SLM. With a higher quality SLM designed in conjunction with the CCD array, we later showed that the information capacity and data transfer rates can be enhanced by many orders of magnitude.

\section{B. Siros Fully Automated Video Demonstration (1995)}

The Stanford demonstration showed that digital encoding techniques could be used to great advantage to improve raw BER, and in combination with error correcting codes digital images could be retrieved with low error rates. The capacity of this demonstration, however, was rather limited. The next step in the development of digital HDSSs accomplished storage of compressed high-resolution video data, requiring substantially larger data storage capacity, and a good BER of about $10^{-8}$. Primarily due to the efforts of J. Heanue, A. Daiber, R. Snyder, and J. Colvin at Siros Technologies, the first video movie was stored and retrieved by recording approximately $5 \mathrm{MB}$ of compressed video data. The optical arrangement was similar to the Stanford demonstration, except that the SLM was a Texas Instruments DMD, with a resolution of $640 \times 480$ pixels. This device overcame all problems of the previously used liquid crystal devices, and allowed us to store the first digital video in a holographic system.

Both video imagery and sound were recorded in $\mathrm{LiNbO}_{3}$ with the MPEG standard using the system of Fig. 56. Data were encoded using $6: 8$ channel codes and a Reed-Solomon $15: 13$ error correction scheme that corrects one byte error per code word. This system was shown on the Discovery Channel in 1995 as part of the Next Step program.

In contrast to the Stanford demonstration, in this experiment the information was permanently stored using ionic fixing. After recording of all data stacks, the crystal was removed from the set up and placed in an oven. It was heated to approximately $120^{\circ} \mathrm{C}$ for $15 \mathrm{~min}$, and then slowly cooled down to room temperature. The thermal fixing efficiency was rather high, approximately $50 \%$ (i.e., the diffraction efficiency after thermal fixing was approximately $50 \%$ of that before the fixing procedure) [77]. Data retrieval in this experiment was implemented in software.

\section{Siros Fully Automated System With Electronic Readout at Video Rates (PRISM, 1996)}

All previous demonstrations were under software control with offline data retrieval. Although it is well recognized that software procedures can often be implemented in electronic hardware, there are many practical issues that make such an implementation far from trivial. At Siros, primarily through the efforts of B. Okas and R. Snyder in cooperation with GTE, Mountain View, CA, as part of the PRISM consortium

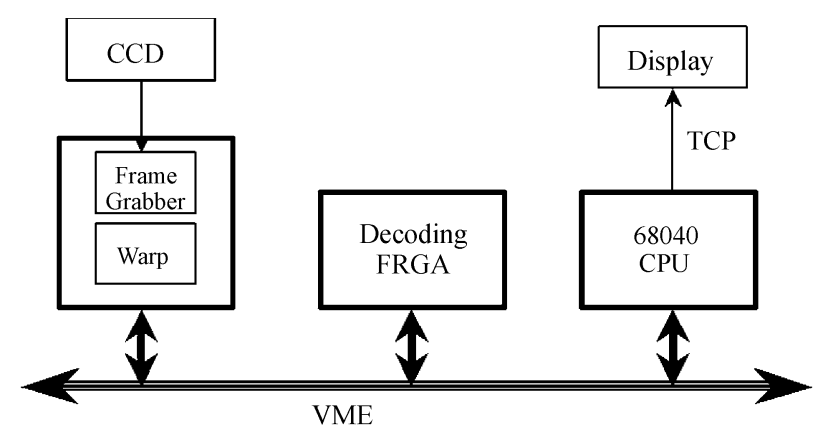

Fig. 57. Electronic block diagram for the Siros real-time video readout system.

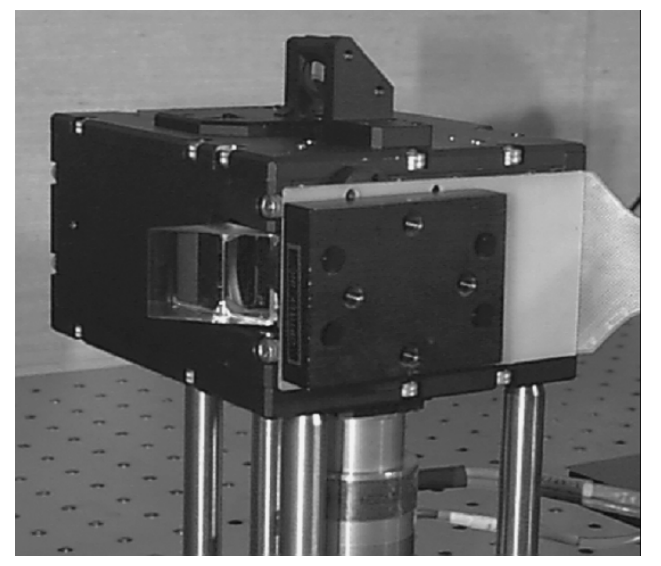

Fig. 58. Siros Technologies compact holographic system.

effort, a fully electronic readout and control system was implemented in 1996. We believe that this was the first demonstration of a fully automated and electronically controlled system. The block diagram of the electronic layout is shown in Fig. 57. The electronic architecture is based on a virtual machine interface (VME) bus. The optical system architecture was the same, as shown in Fig. 56. Temperature fixing in $\mathrm{LiNbO}_{3}$ was implemented for nonvolatile readout, and a total capacity of $5 \mathrm{MB}$ of video data were stored and retrieved at video rates. Channel $6: 8$ coding, ECC Reed-Solomon $13: 15$ coding, bit shuffling, and data warping were all implemented in electronic hardware, as well as overall system control [3]

A small compact demonstration system using $\mathrm{LiNbO}_{3}$ was built primarily by M. McDonald and L. Hesselink at Siros to show that all components could be integrated into an approximately 5.25-in form factor depicted in Fig. 58. This system includes both recording and readout optics and components.

Associative data retrieval capabilities were added to the Siros $90^{\circ}$ demonstration platforms, and content based searches were demonstrated at $20-\mathrm{Gb} / \mathrm{s}$ burst data rates by R. McLeod in 1997.

\section{Stanford University and Siros Fully Electronic Data Readout System Achieving $1 \mathrm{~Gb} / \mathrm{s}$ (HDSS, 1999)}

In previous demonstrations at Stanford, Siros, and IBM, the total system capacity was limited to tens of megabytes, with transfer rates on the order of a few megabytes per second, far from being competitive with the 


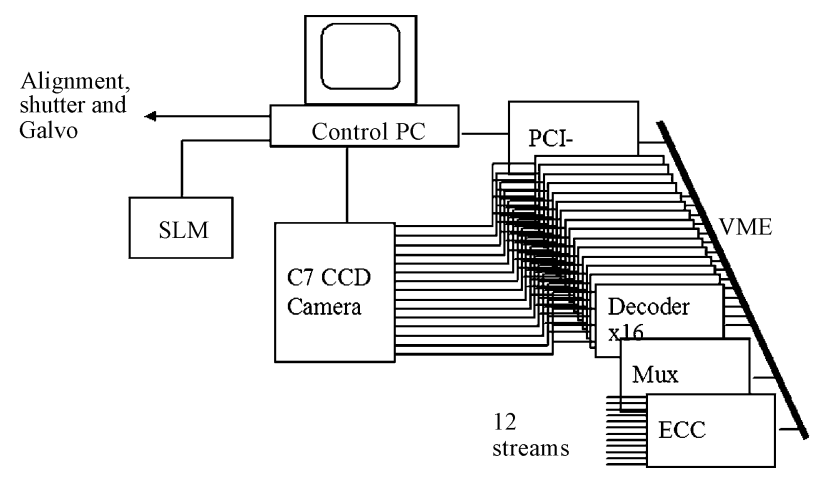

Fig. 59. Electronic block diagram for the $1 \mathrm{~Gb} / \mathrm{s}$ electronics.

then state-of-the-art optical and magnetic drives. The HDSS consortium was formed to develop new components and the systems to demonstrate $1-\mathrm{Gb} / \mathrm{s}$ transfer rate and a capacity of $125 \mathrm{~GB}$ on a 5.25-in disk. A reflective $1000 \times 1000$ element ferroelectric liquid crystal device was designed and built by IBM and Display Tech capable of recording 1000 frames per second. A pixel matched detector array was developed by Kodak, having $1000 \times 1000$ pixels, and a frame rate of $1000 \mathrm{ft} / \mathrm{s}$ using 8-b resolution. The output of this camera was divided into 64 digital channels. A pixel matching very low distortion Fresnel lens was designed and manufactured by Rochester Photonics to achieve less than 0.3 pixel element distortion over the full 1 million pixels in the field of view. A novel random phase code reference beam was coaxially placed with the imaging lens. These components were assembled in the optical set up of Fig. 1. In the first high-speed demonstration, a $90^{\circ} \mathrm{LiNbO}_{3}$ arrangement was implemented using the electronic architecture of Fig. 59. A $6: 8$ channel code and a Reed-Solomon 136:156 ECC for correcting up to ten byte errors in the sequence were used for this demonstration.

A team at Stanford University and Siros consisting primarily of R. Okas, S. Orlov, E. Bjornson, X. Li, F. Dimov, B. Phillips, R. Snyder, D. Kwan, Y. Takashima, and L. Hesselink developed the first implementation of a $1-\mathrm{Gb} / \mathrm{s} \mathrm{elec-}$ tronic readout system. Six data files were recorded, five JPEG images and one counting sequence. The counting sequence was used so that the data could be easily inspected in numerical format. Each source file was divided into two pieces, giving a total of 12 data sequences, corresponding to the 12 output byte streams.

Each recorded hologram stored data at a 55\% efficiency due to $6: 8$ encoding, RS encoding, and masking. Thus, each image of 1048576 pixels corresponds to $576000 \mathrm{~b}$. When this is divided into 12 streams, it gives $6 \mathrm{kB}$ per image per output stream. Thus, our source data files, each of approximately $48 \mathrm{kB}$ (split into two 24-kB sections) required four images to store. The data were stored as four holograms and retrieved with an integration time of $600 \mu \mathrm{s}$, consistent with a 1-ms frame rate. Data passed through the entire system, and the 12 parallel byte streams were collected using the full electronics and checked for accuracy. The raw error rate was $8.7 \times 10^{-5}$.

\section{E. Stanford University and Siros 1-Gb/s Holographic Disk System Demonstrator (2000)}

For thick media like $\mathrm{LiNbO}_{3}$ in the $90^{\circ}$ geometry, capacity is usually limited by media dynamic range and noise rather than by multiplexing crosstalk. For thin media, such as photopolymers, however, this is not the case, and the number of superimposed holograms in a spatial location is largely determined by the limited number of degrees of freedom available for multiplexing. Angular multiplexing in the transmission geometry does not allow sufficient data storage density, and other multiplexing techniques such as shift or peristrophic multiplexing are required. Unfortunately, the displacements necessary for achieving low crosstalk in the shift multiplexing technique are still too large and require the undesirable stop-and-go architecture for the rotating disk when using a CW laser. To avoid this problem, a very sensitive, relatively thick storage medium is required, suitable for pulsed laser recording with nanosecond pulses. Developments in the DARPA-funded PRISM consortium have made great steps forward toward achieving such a medium for recording in the green region of the spectrum using a few hundred milliwatts of power. To achieve recording during constant rotation of the disk, a new multiplexing technique had to be implemented based on a phase-modulated reference beam [12]. In such an architecture, performance can be readily analyzed and compared with more conventional technologies. In the system at Stanford University, densities of over $70 \mathrm{~b} / \mu \mathrm{m} 2$ are reachable for a total system capacity of $125 \mathrm{~GB}$ and a transfer rate of $1 \mathrm{~Gb} / \mathrm{s}$ using the electronics described above. The overall architecture for this system is shown in Fig. 60.

A $1024 \times 1024$ pixel matched $(12.8 \mu \mathrm{m}$ square $) 1000 \mathrm{ft} / \mathrm{s}$ Kodak C7 and IBM LC display are used as a detector and page composer, respectively. Recording and readout is done using a pulsed doubled Nd: YAG laser (532-nm wavelength). A rotating holographic photopolymer disk is mounted on a precision air-bearing spindle. Angular addressing is acheived by using a precision optical shaft encoder (16384 counts per revolution). Different radial positions are addressed via moving the spindle with the mounted disk using a 25-nm-resolution Newport PM600 translation stage. The optical layout for the HDSS WORM demonstration platform is shown in Fig. 61. A sophisticated hardware synchronization is implemented allowing addressing each individual radial location with accuracy of better than $0.1 \mu \mathrm{ms}$, thus allowing (if necessary) incremental hologram recording with multiple pulses of the laser.

The demonstration platform employs a custom-built (by Coastal Optical Systems, Inc., West Palm Beach, FL) short focal length $(17.1 \mathrm{~mm})$ optical relay system [131] which allows 1-megapixel images to be relayed from the SLM to the CCD array with distortions of less than $\pm 1.5 \mu \mathrm{m}$ over the entire SLM format $(13.1 \mathrm{~mm} \times 13.1 \mathrm{~mm})$. The imaging NA for this system is rather high $(0.36)$ while the outside area of the lens is used by the reference beam. (Fig. 61). The spot size in the photopolymer media is $\sim 2 \mathrm{~mm}^{2}$, which corresponds to a raw areal density per hologram of $\sim 0.5 \mathrm{~b} / \mu \mathrm{m}^{2}$. In the current implementation, the speckle correlation shift 


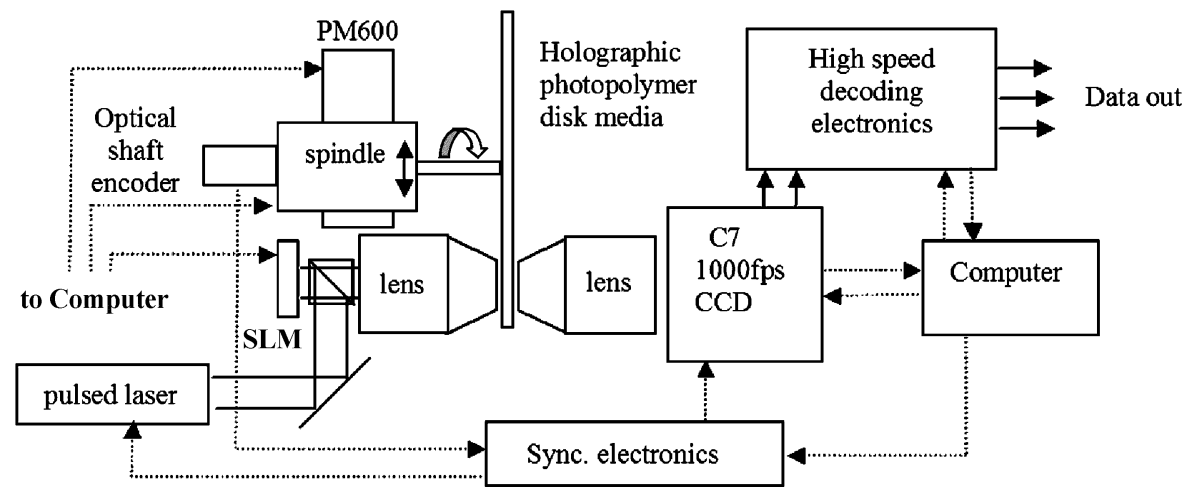

Fig. 60. Overall system layout for the HDSS WORM demonstration platform.

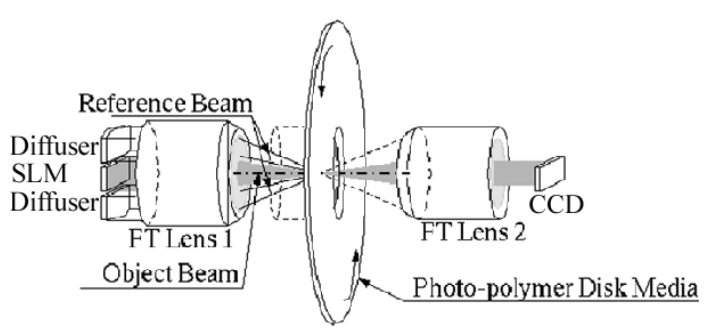

Fig. 61. Optical layout for the HDSS WORM demonstration platform.

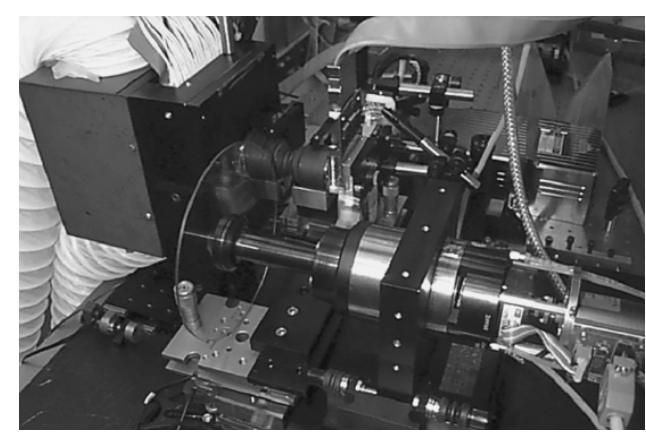

Fig. 62. Photograph of the $1-\mathrm{Gb} / \mathrm{s}$ holographic disk WORM demonstrator.

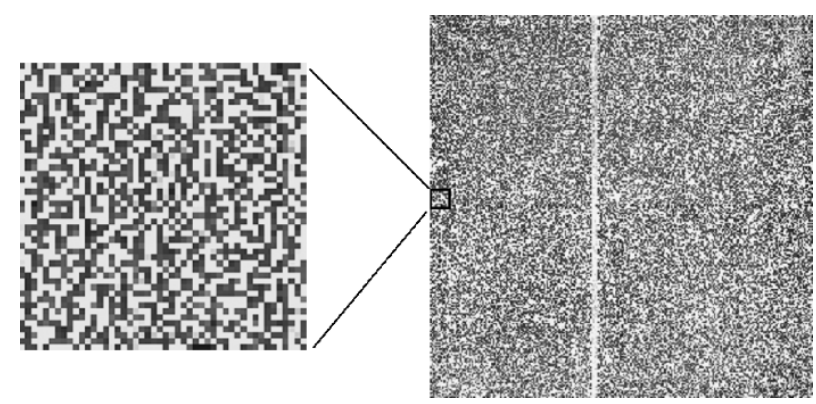

Fig. 63. Sample $1024 \times 1024$ data page $\left(\eta \sim 210^{-3}\right.$, right $)$ and enlarged $48 \times 48$ portion of it (left).

multiplexing technique was used for hologram multiplexing (Fig. 62).

A sample multiplexed hologram recorded in rotating disk media and retrieved with a single laser pulse at $1000 \mathrm{ft} / \mathrm{s}$ is shown in Fig. 63. The raw byte error rate is $<2 \times 10^{-3}$, which provides error-free reconstruction of data with a 136 : 156 Reed-Solomon ECC.
The HDSS holographic disk channel decoding electronics were designed for a sustained data rate of $1 \mathrm{~Gb} / \mathrm{s}$. In the first demonstration (in October 1999), JPEG-encoded color images were stored on the holographic disk and retrieved at 1 $\mathrm{Gb} / \mathrm{s}$ [14], [18]. The source data of 12 images were ECC encoded and converted into holographic channel encoded bitmaps, which were stored in a $200-\mu \mathrm{m}$-thick Polaroid photopolymer disk media. All the retrieved (at $1 \mathrm{~Gb} / \mathrm{s}$ ) data were captured by a logic analyzer and converted back into JPEG format files. Smaples of the original and retrieved images are shown in Fig. 64. The storage density in the first experiment was rather low (approximately $1 \mathrm{~b} / \mu \mathrm{m} 2$ ) and individual holographic page capacity was $65.3 \mathrm{kB}$ (user).

In the later demonstration [132] (Fig. 65), 12 uncompressed video streams were holographic channel encoded, stored in the holographic disk, retrieved at $0.65 \mathrm{~Gb} / \mathrm{s}$, and displayed in real time on three computer screens (four streams per monitor). The total amount of data stored and retrieved was approximately $1 \mathrm{~GB}$ (user) while only $\sim 10 \%$ of the area of a 6.5-in-diameter photopolymer disk was used. The media thickness was $200 \mu \mathrm{m}$.

\section{F. Stanford Ultrahigh $>10-G b / s$ Optical Data Rate Demonstration (2000)}

In the holographic disk system at $1 \mathrm{~Gb} / \mathrm{s}$, the hologram signal strength largely exceeds the camera noise. Thus, the holograms can be read at much higher optical data rates provided that the holograms can be physically transported under the optical readout head at sufficient and sustainable rates. The latter depends on the recording density and the disk linear velocity.

In later experiments, we achieved sustained optical data rates as high as $10 \mathrm{~Gb} / \mathrm{s}$ [17] by increasing the optical efficiency of the optical delivery system. The storage density in these experiments was approximately $10 \mathrm{~b} / \mu \mathrm{m} 2$. A sample hologram read out at $10 \mathrm{~Gb} / \mathrm{s}$ is shown in Fig. 66 .

\section{G. Tamarack Multistore Jukebox}

In the mid-1980s, MCC, in collaboration with Stanford University, started a holographic data storage program in response to Japan's Fifth-Generation Computer Program. The objective of the program was to address the issue of data access latency caused by rotating disk drives. As processor 

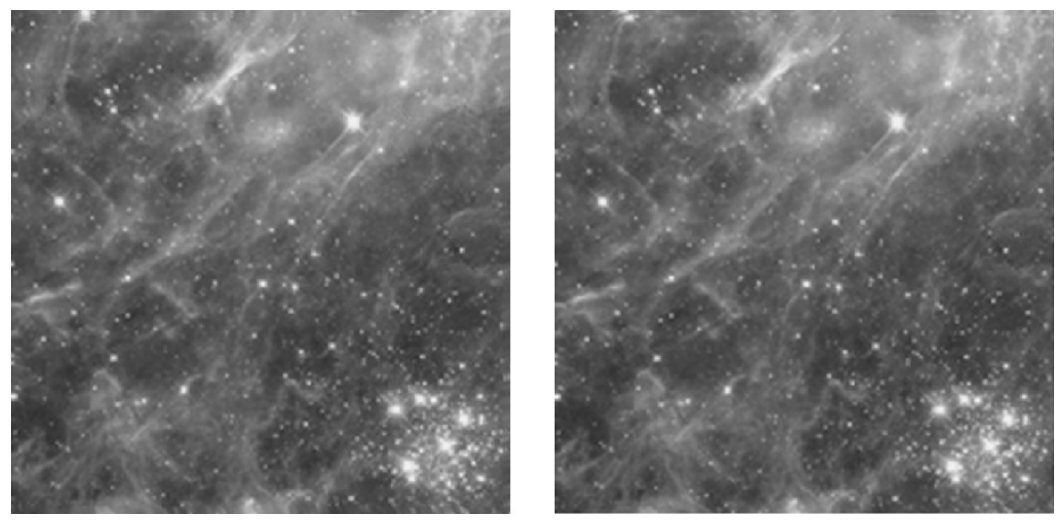

Fig. 64. Retrieved (left) and original (right) images.

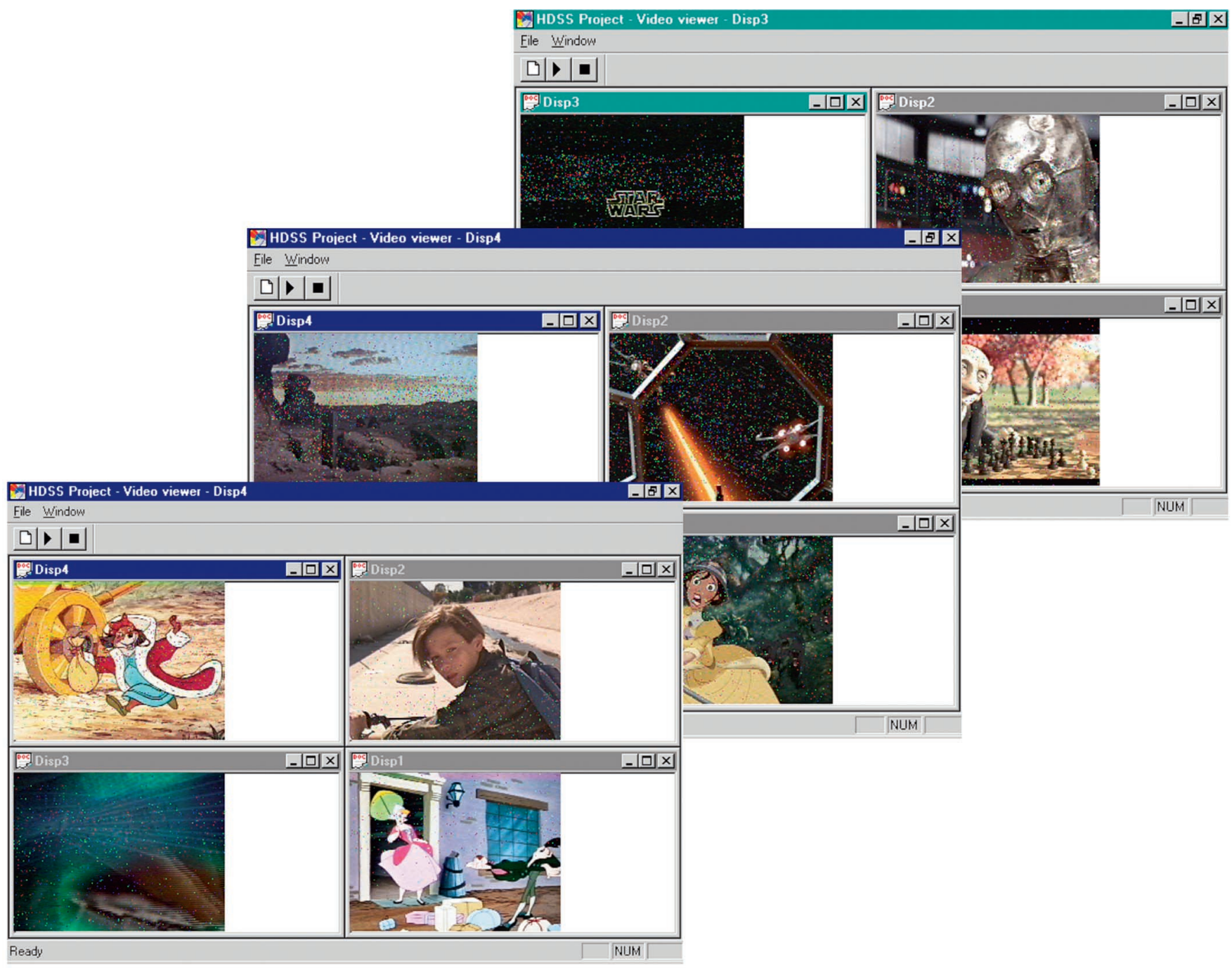

Fig. 65. Twelve uncompressed video streams display.

speeds advance, the I/O gap-the latency of getting access to data once a command for it has been issued-increases. HDSSs could potentially solve this problem as they do not require moving parts and, therefore, can provide much shorter access times, on the order of microseconds.

The MCC program culminated in the formation of Tamarack storage systems, a spinoff from MCC formed in 1992 [23]. Tamarack targeted the development of a disk jukebox containing 30 disks and a 5.25-in form factor drive. An excellent mechanical system was developed, but unfortunately its holographic performance target of $30 \mathrm{~GB}$ was not demonstrated and thought to be too small to be attractive in the mid-1990s. A lack of a suitable material ultimately lead to the demise of Tamarack. A photograph of the advanced Tamarack holographic optical head assemby is shown in Fig. 67. 


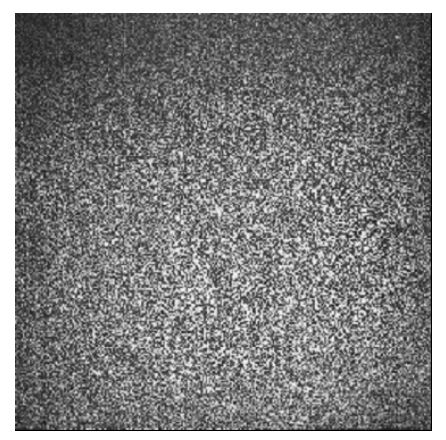

Fig. 66. Sample hologram reconstructed at $10 \mathrm{~Gb} / \mathrm{s}$.

\section{H. IBM Holographic Material and System Testers}

A research team at IBM Almaden, San Jose, CA, has built three holographic digital data storage test platforms [133].

The PRISM tester [57] shown in Fig. 68 was designed and built for the purpose of having a stable platform for testing holographic data storage materials. It was completed in 1996 and has provided an important means for testing a wide variety of materials ranging from photopolymers to inorganic photorefractive crystals. Some of the results of these measurements are shown in Fig. 32. Two additional demonstration systems were also built at IBM, the DEMON I and II in 1997, shown in Figs. 69 and 70.

These platforms were used to study coding approaches [123] and to study the performance of holographic systems under various conditions. For example, IBM also stored videos similar to the earlier Siros demonstrations and achieved very high data storage densities in DEMON II using $\mathrm{LiNbO}_{3}$ as a recording medium [30].

Important optical approaches were developed and tested for achieving flattop intensity profiles for the image bearing signal beam [134] as well as methods for reducing the central spike in the object beam at the Fourier transform plane [133]. Additionally, associative retrieval methods were developed which proved the superior performance of holographic-based searches over traditional digital computer searches, as described above.

\section{Rockwell Ultrashort Access Time Testbed}

Ultrashort data access times were achieved by a team of researchers at Rockwell International Research Center, Thousand Oaks, CA, as part of the HDSS program. Using acoustooptic beam deflectors for the reference beam, access times of less than $50 \mu$ s were demonstrated in $\mathrm{LiNbO}_{3}$ in the $90^{\circ}$ configuration, as shown in Figs. 71 and 72 [5], [135]. Short access times combined with associative data retrieval could provide an interesting market opportunity, as other storage devices based on rotating disks have access times of a few milliseconds and cannot contain as much data under the head. In particular, for searching video databases, such capability could be valuable.

\section{J. Caltech and Holoplex Read-Only Memories and Replication System}

Digitial holographic disk systems based on polymer materials were also demonstrated by Holoplex, Pasadena, CA, and at Caltech in the late 1990s [136]. The demonstration system employed 100- $\mu$ m-thick Dupont photopolymer and a combination of angular and peristrophic multiplexing. The recording step was performed using a $\mathrm{CW}$ laser and stationary disk. The reported density was approximately $40 \mathrm{~b} / \mu \mathrm{m}^{2}$ while the channel decoding was performed by software. The special emphasis of this work was on development and demonstration of holographic disk replication technology, where the data from a prerecorded master disk was transferred to a replica disk via simultaneous readout of holograms from the master and recording onto the replica. Schematically, the setup is shown in Fig. 73 and a photograph of a ten-beam replicator is shown in Fig. 74. Prior to demonstrating digital holographic systems, much pioneering work was carried out at Caltech relating to system architecture issues, as described above.

\section{K. Optware Colinear Holography System}

Optware Corporation, Yokohama, Japan, is developing a colinear holographic system, as shown in Fig. 75. The system involves a two-wavelength approach in which a red laser is used for servoing and a green laser for holographic storage. The green 532-nm laser is used for holographic reading and writing, while the red laser is used for tracking and focusing. A dichhroic beam splitter combines the two beams onto the holographic medium. The Texas Instruments DMD is used as a page composer. The CMOS detector has a $3 \times 3$ oversampling ratio for each data pixel. The object and reference beam are colinear at the medium, and both reflect from the lower surface of the recording medium. Polarization optics are used to efficiently utilize available laser power. The size of the holograms on the bottom surface of the photopolymer medium is approximately $200 \mu \mathrm{m}$, which is typically $400-500 \mu \mathrm{m}$ thick. Reported data densities are approximately $40-50 \mathrm{~b} / \mu \mathrm{m}^{2}$.

The interesting aspect of this approach is the symmetrical Bragg selectivity in both the track and radial direction affording close packing of holograms, which is not easily achievable using an off-axis reference beam geometry. Since the object and reference beam pass through the medium twice, signals are enhanced, but noise levels build up as well. The key research and development issue for this approach is to reduce noise through reduction of low-frequency media response and improvement in signal strength by increasing the thickness and reducing the inherent media scatter noise while maintaining a low shrinkage rate. The reported media for this development effort are produced by Aprilis, Inc., Maynard, MA, and Inphase, Inc., Longmont, CO.

\section{Aprilis High-Density CROP Disk Testbed}

Aprilis, Inc., a spinoff company from Polaroid, has been at the forefront of materials development based on CROPs. Recently [137], significant advances have been reported 


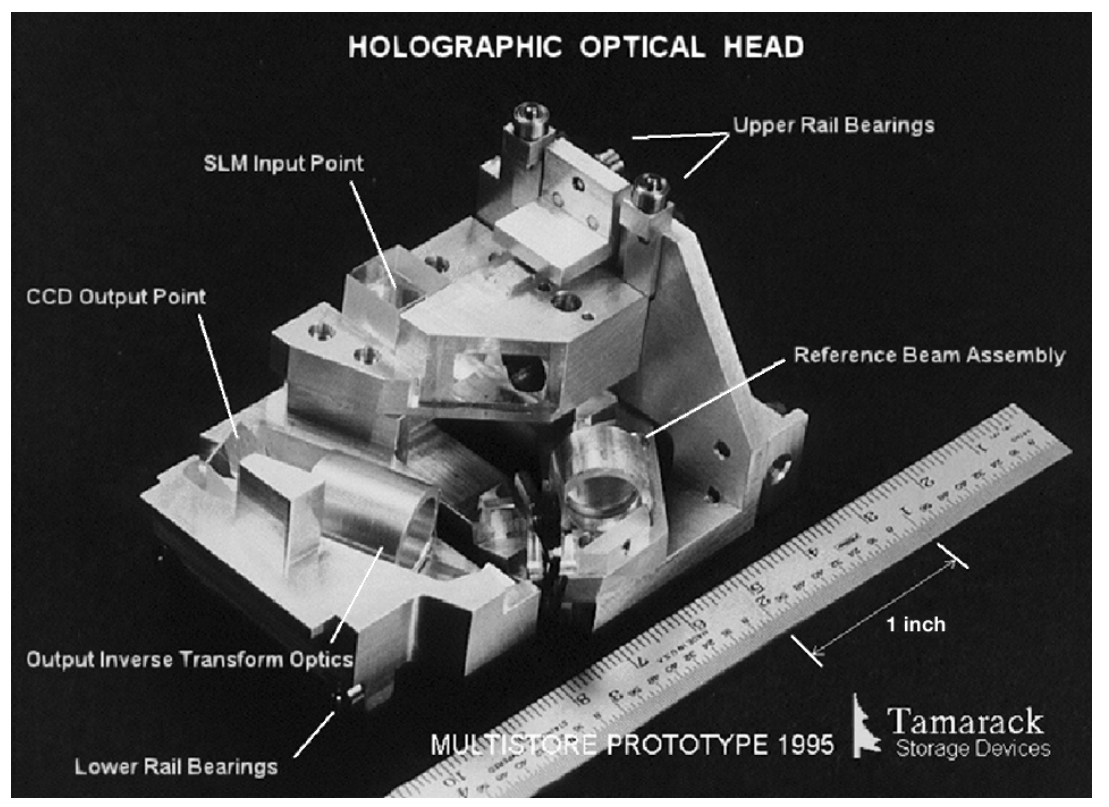

Fig. 67. Photograph of the Tamarack optical head system (with permission).

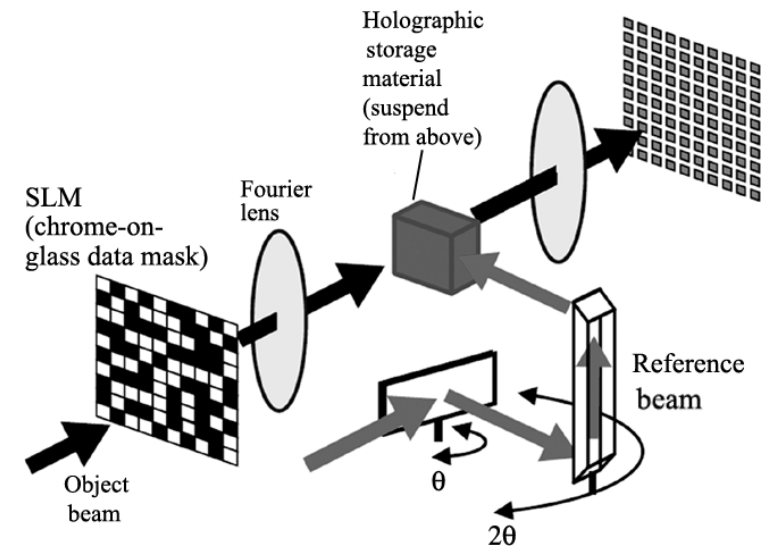

Fig. 68. The primary features of the IBM PRISM holograhic materials test apparatus. The SLM is a chrome-on-glass mask, and the detector array is a $1024 \times 1024$ of a large CCD camera. A pair of precision rotation stages allow the reference beam to enter the storage medium under test at any horizontal incidence angle (courtesy IBM, with permission).

with improved materials that are capable of supporting digital data densities of $150 \mathrm{~b} / \mu \mathrm{m}^{2}$ in 400 - $\mu \mathrm{m}$-thick media, corresponding to approximately $150 \mathrm{~GB}$ on a 5.25 -in disk. The raw BER for 262-kb data pages was approximately $5.510^{-3}$, which can be significantly reduced to better than $10^{-15}$ with the use of error correction codes as described earlier. The dynamic range of the medium was large with an $M \#$ of approximately 22 . The media sensitivity is high with a peak initial value of $6.75 \mathrm{~cm} / \mathrm{mJ}$ reducing to $0.5 \mathrm{~cm} / \mathrm{mJ}$ for attaining $87 \%$ of the available dynamic range or accumulative grating strength. Approximately 155 holograms are recorded in the same spatial location using angular and peristrophic [9] multiplexing. Peristrophic multiplexing is a method for recording holograms in the same physical location by rotating the reference beam with respect to the recording medium as well as by rotating the medium about an axis normal to the surface of the medium. A typical result is shown in Fig. 76, where the histogram of bits is shown for superposition of 155 holograms in Aprilis HMD CROP media. The growth of the cumulative grating strength and reduction in sensitivity as a function of total accumulated exposure is shown in Fig. 77. In a recent experiment, as yet unpublished, Aprilis has achieved 250-GB storage capacity on a single disk, indicating that the Aprilis HMD CROP material is currently the best available (based on published data) medium because of its high sensitivity, large dynamic range, and low shrinkage.

\section{InPhase Technologies Demonstration Platform}

InPhase Technologies has been developing photopolymer media based on technology originally conceived and practiced at Bell Laboratories-a two-chemistry medium composed of two independently polymerizable and compatible chemical systems consisting of low-refractive index matrix precursors and high-refractive index polymerizable monomers [138].

The two-chemistry system allows in situ formation of the background matrix and optimization of various recording chemistries to optimize dynamic range, sensitivity and shrinkage. $M \#$ s of 6.7 have been reported, which compares favorably with $\mathrm{LiNbO}_{3}$, which has a similar dynamic range, but lower sensitivity of one to two orders of magnitude. The medium shrinks, however, by approximately $0.1 \%$, reducing the usable dynamic range for digital storage as compared with $\mathrm{LiNbO}_{3}$ which is a solid and very dimensionally stable. Recent advances by InPhase indicate that shelf life can exceed years, and holograms retain their strength and fidelity for at least $1000 \mathrm{~h}$ of exposure. Media thickness can reportedly also be increased to at least $1.5 \mathrm{~mm}$, but no test data have been made publicly available. For thicker photopolymers, shrinkage is a serious problem, and its management requires tradeoffs between sensitivity, dynamic range, and shrinkage. Theoretically, the $M \#$ or dynamic range should 


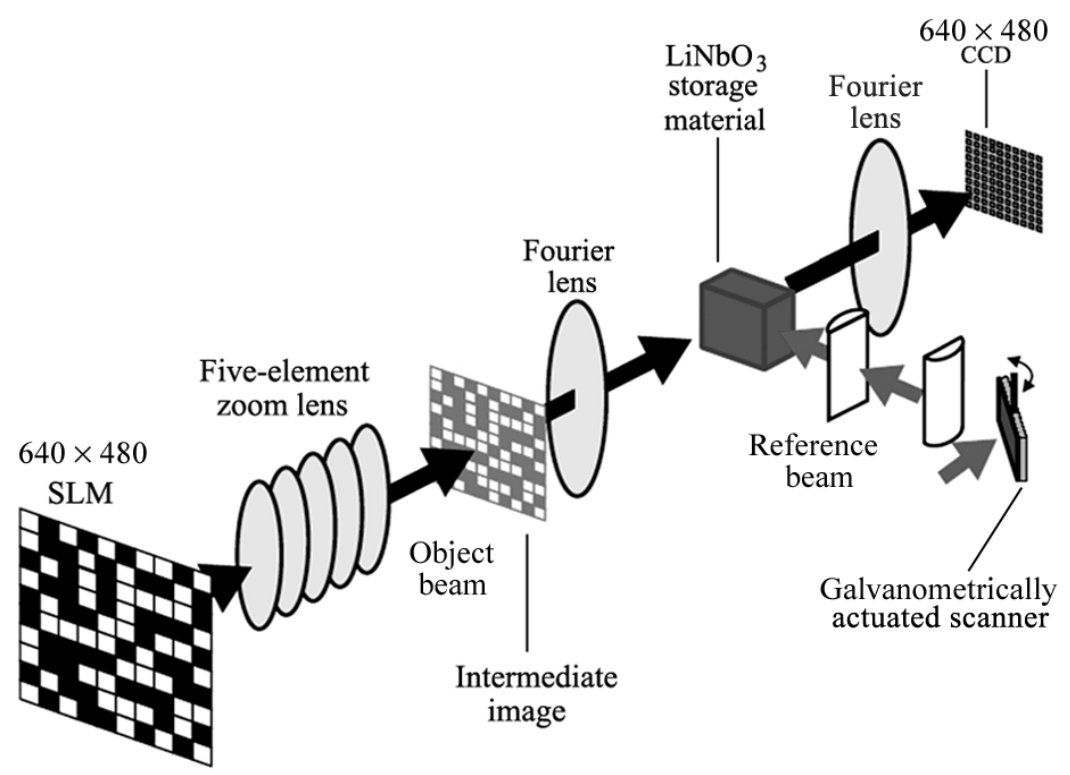

Fig. 69. Salient features of the DEMON I holographic digital data storage engine. A five-element zoom lens demagnifies the SLM image to an intermediate image plane, which is then imaged onto the $\mathrm{CCD}$ detector array with a pair of lenses. This tester was designed for use with $\mathrm{LiNbO}_{3}$ media (Courtesy IBM, with permission).

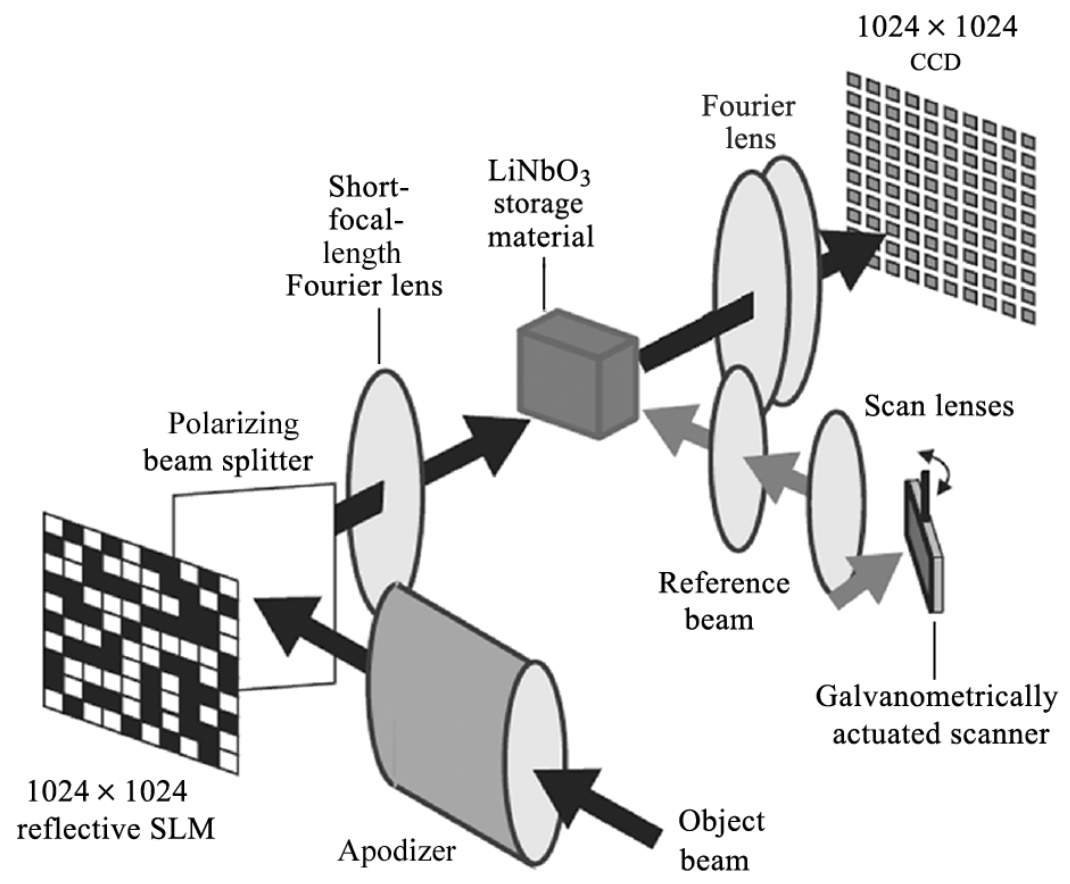

Fig. 70. Primary features of the DEMON II holographic digital data storage engine. This system has demonstrated over $100 \mathrm{~b} / \mu \mathrm{m}^{2}$ (courtesy IBM, with permission).

increase quadratically with thickness, but as a result of these tradeoffs, in practice the useful dynamic range is often much smaller. At higher media thicknesses, keeping optical distortions to a small fraction, typically $1 / 4$ to $1 / 10$ of the wavelength of light, presents a challenge. InPhase indicates that a proprietary technique allows them to manage optical distortions efficiently during the manufacturing process. A complete system demonstration incorporating media with thicknesses exceeding $1 \mathrm{~mm}$ has not yet been reported, however.

The InPhase media has been tested in a demonstration platform, indicated in Fig. 78, where a typical rotating disk ge- ometry is used, as shown. The reference beam is modulated in phase to produce a speckle pattern at the disk surface, giving rise to small required displacement for multiplexing, as described above in the section on speckle multiplexing.

Using a $750-\mu \mathrm{m}$-thick medium, a user bit density of $35 \mathrm{~b} / \mu \mathrm{m}^{2}$ been reported. Media capable of higher densities are under development at InPhase, but no published data describing media performance are avaialble. The reported recording density of $\sim 45 \mathrm{~b} / \mu \mathrm{m}^{2}$ was achieved in $750-\mu$ m-thick media and correlation shift multiplexing at relatively low data rates (a few megabits per second) [116]. 


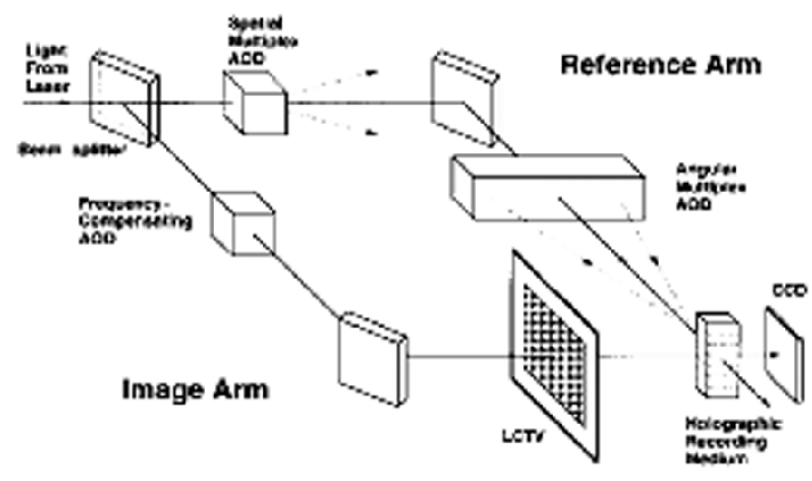

Fig. 71. Basic architecture as used by Rockwell for demonstrating access times around $30 \mu \mathrm{s}$ (with permission).

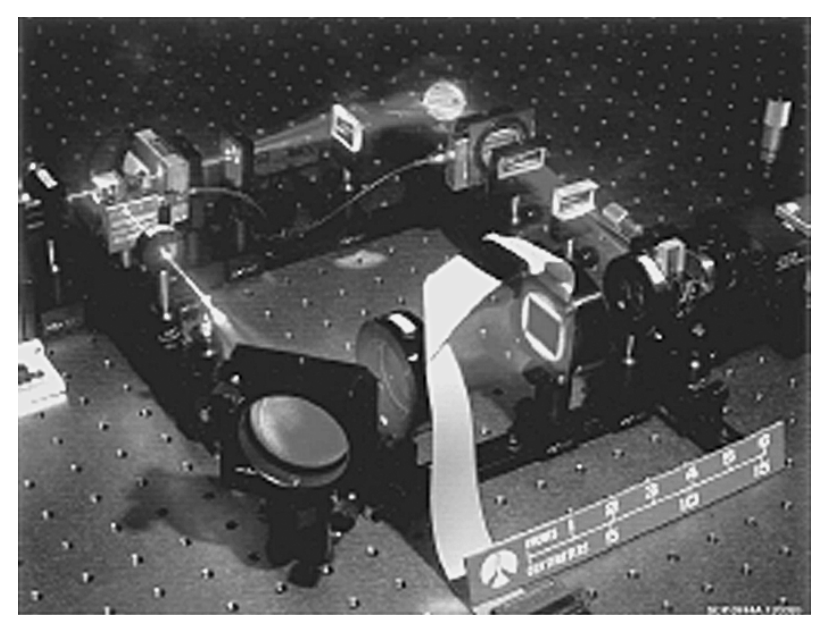

Fig. 72. Photograph of the Rockwell holographic fast access storage demonstrator.

Issues related to improvement of material shrinkage and media recording sensitivity are currently under investigation for this photopolymer material system.

\section{N. Single-Bit Holographic Storage}

Storing single bits inside a holographic medium in layers at various depths has been demonstrated in $\mathrm{LiNbO}_{3}$ and photopolymers. In analogy with the multilayer CD and DVD optical systems, bits are stored inside the medium on physical layers [139] or virtual layers [140] and read out with a focused laser beam capable of addressing layers in depth. Data are stored by interfering two counterpropagating focused object and reference laser beams, as schematically shown in Fig. 79, as reported by [141].

In the recording region, small reflection holograms are formed, which can be read out using a single laser beam. Each hologram can represent a bit, or multiple bits can be represented by elongated holographic marks in the direction perpendicular to the optical axis to produce the equivalent of a DVD pit, where the pit length encodes data by measuring readout signal transitions from low to high reflectivity, and vice versa. Multiple single-bit holograms can be superimposed to record multiple bits in the same physical location. By combining wavelength, angle multiplexing, and depth multiplexing (i.e., recording holograms at different depth locations), total storage capacity $S$ is equal to

$$
S=m_{L} \cdot m_{\lambda} \cdot m_{\theta} \cdot S_{A}
$$

where $m_{L}$ denotes the number of layers, $m_{\lambda}$ represents the number of wavelength-multiplexed holograms, $m_{\theta}$ is the number of angle-multiplexed holograms, and $S_{A}$ represents the surface bit density. A capacity of $100 \mathrm{~GB}$ is, in principle, achievable using two layers, ten wavelengths and 16 different angles for multiplexing a total of 160 holograms per location, using a bit spot size of $1 \mu \mathrm{m}$ radius [141]. A widely tunable laser is needed, as the wavelength selectivity is relatively small, requiring tens of micrometers of wavelength separation between multiplexed holograms. Such semiconductor lasers do not currently exist, but, for example, dye lasers can be scanned over a wide range of wavelengths. The angular separation for good Bragg selectivity is also quite large, as angular selectivity is at a minimum for the reflection geometry, allowing only a few tens of holograms to be multiplexed. In practice, storage densities not much higher than a few gigabytes per disk have been obtained.

Siros Technologies investigated an alternative approach using only a single hologram per location per layer, on multiple virtual layers in a homogeneous medium, and by using confocal detection for readout. The layers are called virtual because they are created during the recording process by writing bits at particular depths. A schematic arrangement is shown in Fig. 80.

Data are recorded as show in Fig. 79 using a green laser. A second red laser is used for servoing and focusing. The two laser systems are dynamically linked using a fast acting optoelectronic servoing system. The servo layer is similar to a grooved DVD recordable medium layer, which is used to guide the writing beam during recording of the information. A split focusing and tracking detector is combined with a pinhole to achieve confocal detection allowing a layer spacing of less than $10 \mu \mathrm{m}$ for light with a wavelength of $532 \mathrm{~nm}$. This arrangement allows a maximum of 10-15 layers to be packed closely together without the need for spherical aberration correction using an NA $\sim 0.5$ for the recording and readout beam. Embedded data in each layer allows fast in-plane and vertical seeks [142]. Experimental results for a nine virtuallayer disk using Aprilis photopolymer media and a green 532-nm laser are shown in Fig. 81. As signal strength is typically 10x smaller than for DVD systems, i.e., $1 \%$ versus $20 \%$, respectively, high-gain low-noise amplifiers are used for data readout. Quantitative system performance measurements have not been reported, but initial results appear rather promising [142].

Theoretical calculations indicate that a medium containing 40-50 stratified layers might be possible using a red 658-nm laser and an NA $\sim 0.5$. Shorter laser wavelengths allow closer layer spacing and increased capacity. For example, a blue laser having a wavelength of $405 \mathrm{~nm}$ and a focusing lens of NA $=0.85$ allows 25 channel $\mathrm{b} / \mu \mathrm{m}^{2}$ to be recorded on BlueRay DVD disks. Assuming a reduced density of 15 channel $\mathrm{b} / \mu \mathrm{m}^{2}$, as holographic bits will not have the same sharp edges as data bits recorded on phase change media, 


\section{REPLICATION}

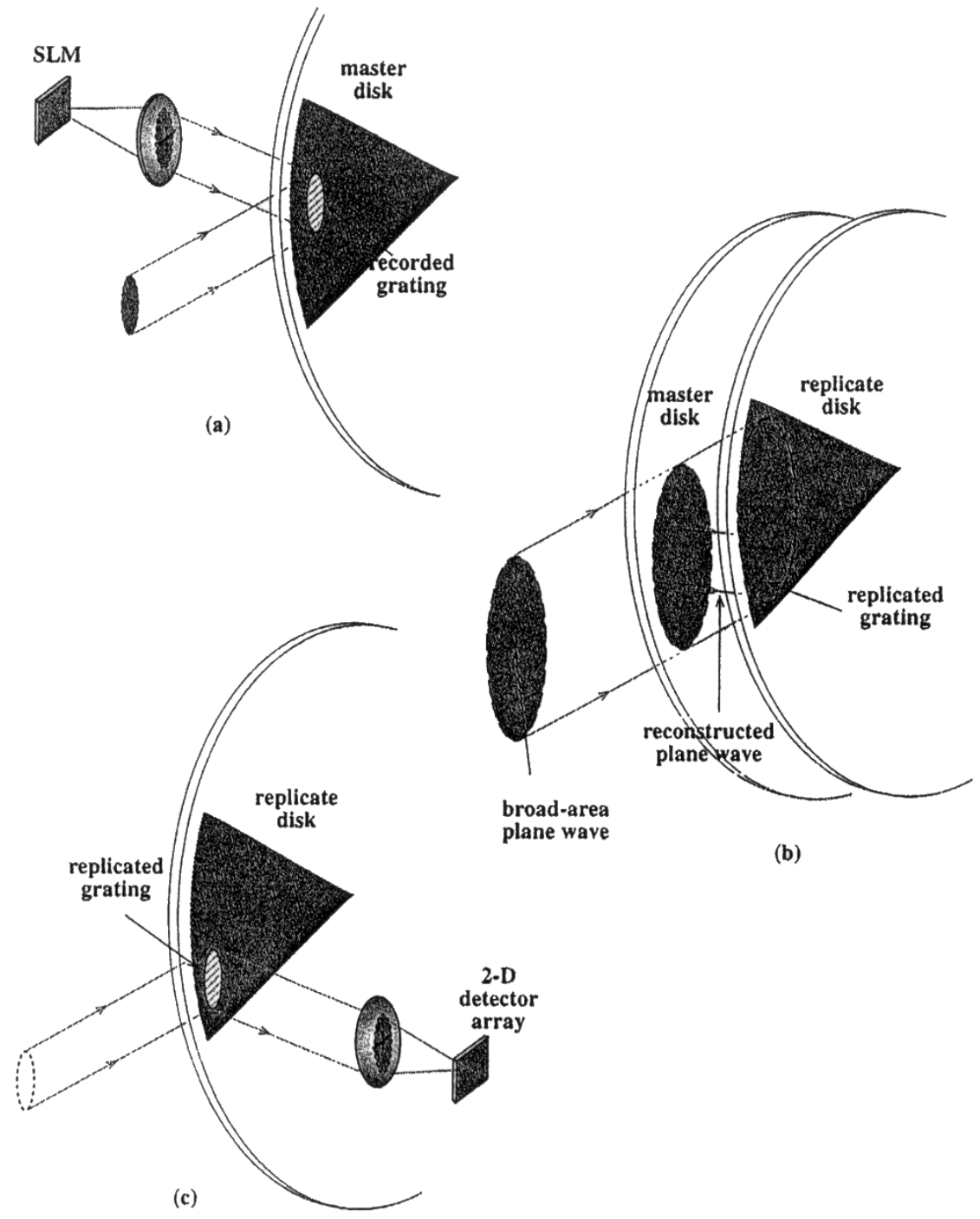

Fig. 73. Schematic of Holoplex repliactor for recording, replicating, and reading a hologram (with permission).

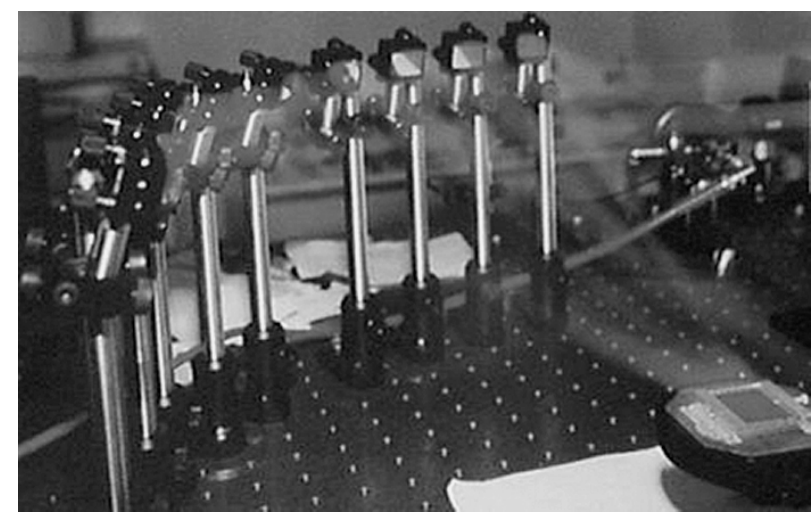

Fig. 74. Photograph of ten-beam Holoplex replicator (courtesy Holoplex, with permission).

and allowing for lower signal strength of the holograms (around $1 \%$ ) versus $20 \%$ for DVD signals, a density of at least 150 channel $\mathrm{b} / \mu \mathrm{m}^{2}$ or $150 \mathrm{~GB}$ per disk should be achievable. Theoretical calculations show that for a signal having a $>40-\mathrm{dB}$ SNR, 40-50 layers might be possible in a single medium, giving rise to capacities of approaching 1 TB per disk. Data transfer rates can be increased by using multiple beam readout, a technique developed by Zen Research, Inc. Seven to ten bits can be read out simultaneously, providing data transfer rates approaching $1 \mathrm{~Gb} / \mathrm{s}$.

\section{OUTLOOK}

While there has been tremendous progress in research into HDSSs over the last decade, there is still no mass-produced or custom-built product available based on this technology. The primary reason is that continued incremental advances in magnetic and optical storage have met the needs for most applications and have generated whole industries in which 


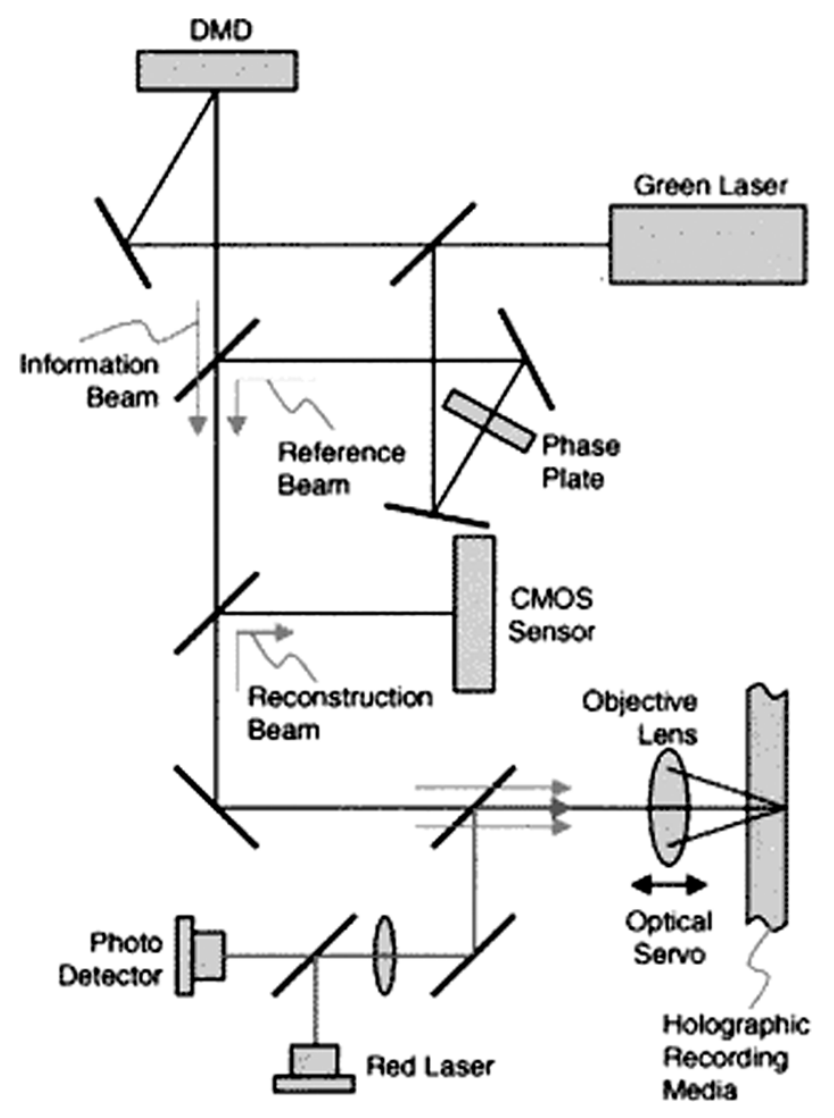

Fig. 75. Optical arangement of the Optware media tester (courtesy Optware, with permission).

component vendors are strongly synchronized to the needs of the system integrators that provide the commercial products.

Although we continue to see advances in the components and materials for HDSSs, the bar for market entry is raised each year as the incremental advances in traditional storage systems continue. As far as mass production is concerned, until we begin to see a significant plateau in the advancement of the traditional technologies, they will continue to dominate. There may, however, be custom applications in which the high performance of HDSSs can be a technology discriminator. One such example is associative data retrieval, in particular for image-rich databases. Traditional search approaches require some form of content indexing, which is difficult to do with pictures of objects that vary in size, orientation and completeness of description throughout the database. To automatically find a person, from an incomplete or fuzzy description, among thousands of people in a 60-min video movie with better than $80 \%$ accuracy, for example, is currently beyond the capability of commercial search engines. Holography is inherently suited for solving this problem, and it might provide the impetus for further commercialization efforts. The ultra short access times of a solid-state holographic system are also attractive for improving computer performance by reducing latency.

A leading challenge in commercializing HDSSs is developing suitable materials. Compact crystals generally require sophisticated components and optical system engineering; these materials have not yet been reduced to a technology that results in repeatable fabrication of media having the properties required for a system. Tens of tons of $\mathrm{LiNbO}_{3}$, however, are annual produced at relatively low cost for signal processing applications. At the same time, however, recent demonstrations by Aprilis of their HMD product indicate that capacities of over $250 \mathrm{~GB}$ per disk are feasible. This benchmark provides an important milestone toward a serious commercialization effort. InPhase also has made good progress in creating thick, high-quality optical media. Photopolymers lend themselves well to disk-based architectures that could leverage much of the mechanical technology used in current disk drives and lend themselves well to mass production. Although at present photopolymers have not yet matured to the point where they can consistently provide the desired properties and reliability, a well-designed and executed commercialization effort could provide the know-how needed to achieve commercial quality media in a relatively short period.

Materials used for holographic data storage are generally specifically developed for this type of system. To date, HDSSs have not been able to leverage the development of materials for other purposes due to the tight material requirements enforced by system requirements. Increasingly, however, research into crystals and photopolymers for photonic networking and interconnection has received significant interest, and may result in materials that, with appropriate modification, are suitable for holographic data storage.

The various optical and electrical HDSS components used are adapted from components used in other industries. SLMs are a significant driver in the display industry. The Texas Instruments DMD is an extremely high-performance device capable of allowing data rates of $1 \mathrm{~Gb} / \mathrm{s}$ with very high contrast digital data pages. CCD and CMOS detector arrays have numerous applications in image capture, including digital photography and manufacturing inspection. Size, data rate, and performance of these detectors is close to what is required for a holographic system. Advances in electronics in the telecom and storage industries may also support HDSSs. Laser sources continue to improve incrementally and new technology for tunable lasers developed for the telecom industry may be applicable to holographic data storage. These may enable significant progress in research into wavelength multiplexing architectures. Additionally, the optical storage industry is developing high power blue lasers that would be excellent enablers for holographic storage. Once significant, tangible commercial opportunities mature that current technologies cannot economically capture and for which HDSSs are genuine candidate solutions, we can expect significant synchronization of these various efforts to mature the technology for mass production and custom development.

What can we then expect in the future? Several different scenarios come to mind, all taking advantage of the unique properties of holographic systems, namely, the high data storage density, short access times, superior data transfer rates, and ultrafast search capability. Schematically, four potentiality different scenarios might play out for future 


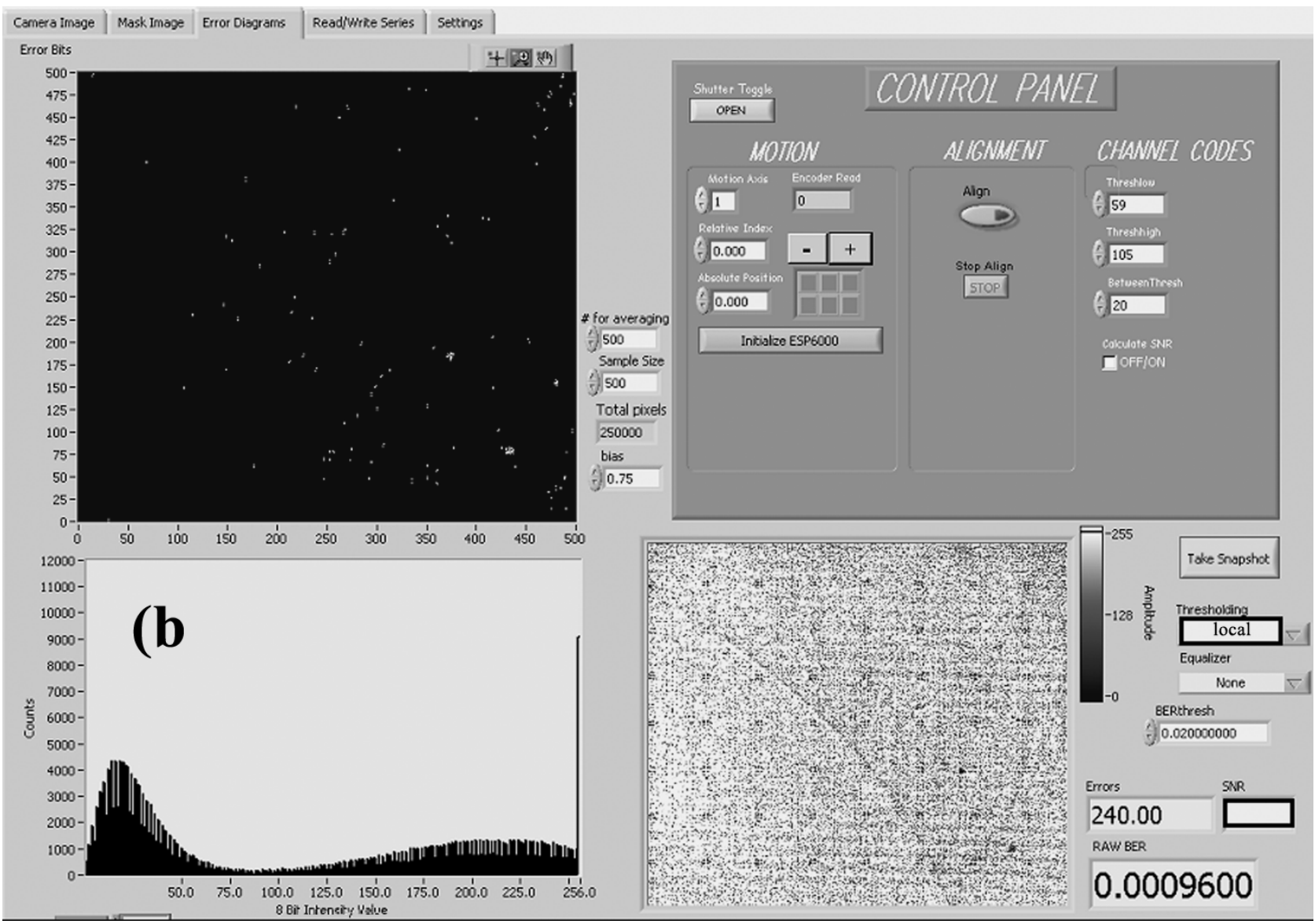

Fig. 76. Reconstructed $262-\mathrm{kb}$ digital data page on CCD detector with $2 / 1$ oversampling, direct error map of page, histogram distribution of ones and zeros with application of local threshold ontensities, and raw BER of the 60th of 155 colocationally multiplexed holograms, each recorded at a fractional Fourier transform plane using peristrophic and planar-angular multiplexing in Aprilis HMD CROP media with 400- $\mu$ m-thick material using an Nd: YAG laser at $532 \mathrm{~nm}$ (courtesy Aprilis, with permission).

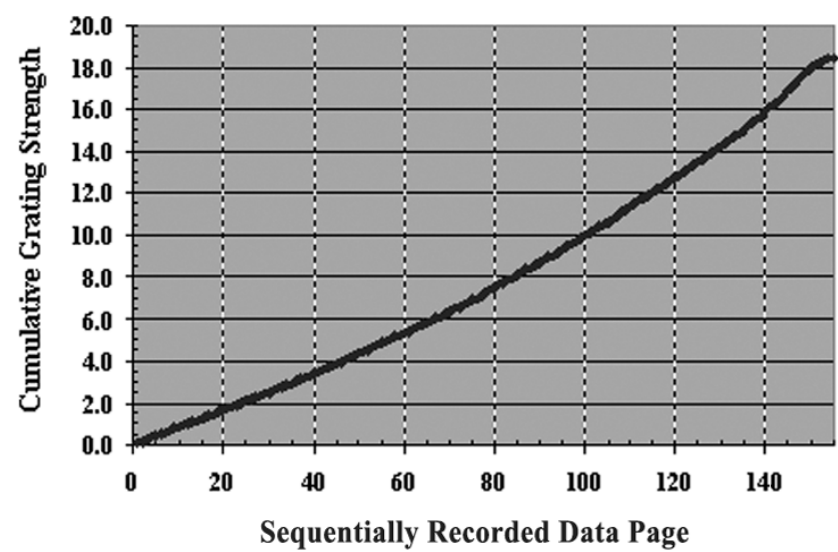

(a)

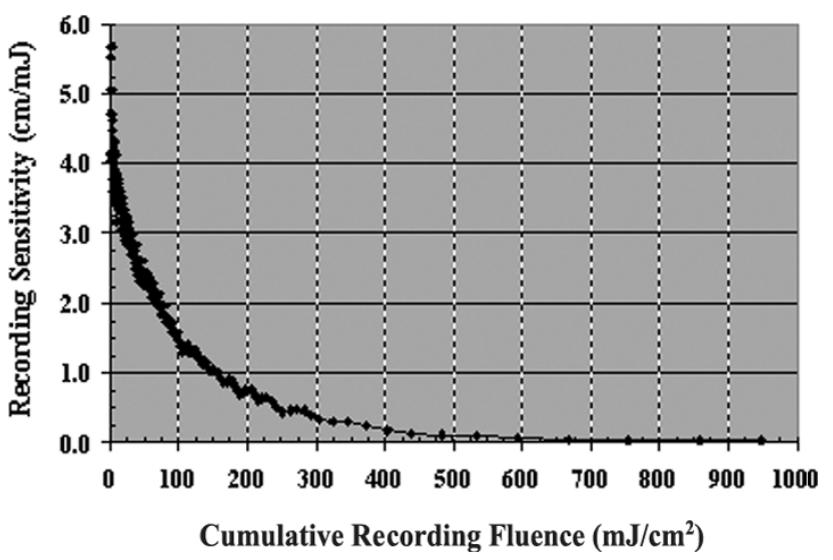

(b)

Fig. 77. (a) Growth of accumulative grating strength in Aprilis HMD CROP material. (b)

Reduction in sensitivity as function of recording fluence in Aprilis HMD CROP material (courtesy

Aprilis, with permission).

commercialization efforts [1], as shown in Fig. 82, published by IBM.

Photopolymer systems, either based on the CROP technology from Aprilis or the two-chemistry technology from InPhase, appear destined to be potential candidates for DVD follow-on products. Both materials exhibit characteristics that make them close to suitable for commercialization. Capacities of over $250 \mathrm{~GB}$ per disk were demonstrated by Aprilis using their HDM media, and at Stanford University, transfer rates exceeding $10 \mathrm{~Gb} / \mathrm{s}$ were measured in a fully functional testbed using an earlier developed Aprilis CROP photopolymer medium. There are, however, critical tests that 


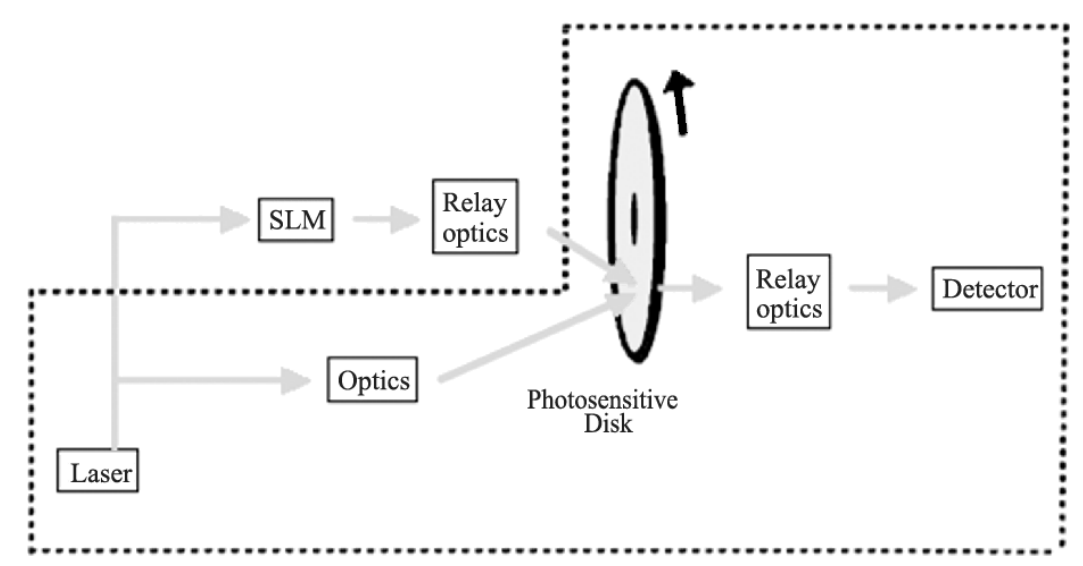

Fig. 78. Block diagram of the InPhase holographic data storage demonstrator (courtesy InPhase, with permission).

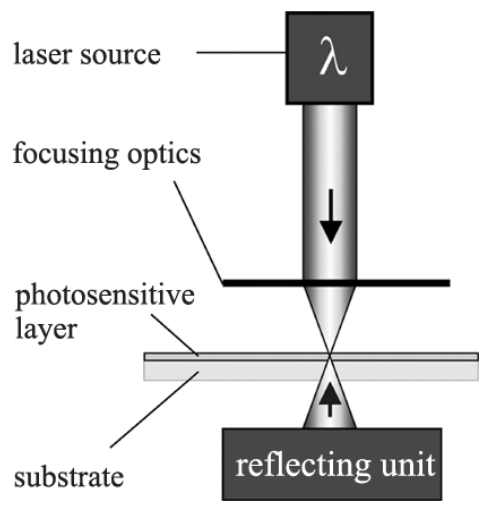

Fig. 79. Single-bit hologram recording configuration (courtesy Eichler, with permission).

still must be performed. Even although media development has made significant progress over the past ten years, there is still no definitive test in which both density and transfer rates are measured in a working prototype, having performance characteristics that are commensurate with the optical data storage roadmap shown in Fig. 1, i.e., capacities exceeding $200 \mathrm{~GB}$ on a disk and transfer rates of $500 \mathrm{Mb} / \mathrm{s}$ or higher. As holographic diffraction efficiency is inversely proportional to the square of the number of superimposed holograms, capacity and transfer rate are tradeoff parameters. Increasing one goes at the expense of the other one. Additionally, tolerances and systems issues require tradeoffs that lower the attainable capacity and transfer rates of the system further. It is, therefore, critical that in future system tests, total system performance is measured, similar to what was accomplished in the Stanford HDSS consortium demonstration, as a prerequisite to full scale commercialization.

Another critical question in HDSS development is the amount of laser power that will be available at a reasonable cost. Currently all demonstration testbeds have used powerful, expensive pulsed solid-state laser sources. Solid-state lasers are costly and require large amounts of electrical power. Fundamentally, the large required laser power is caused by low diffraction efficiency and high data rates. When normalized to photons per bit, almost all optical storage systems require about the same energy on the detector per bit, for writing and reading, $\sim 100 \mathrm{fJ} / \mathrm{b}$ for $\mathrm{CCD}$ arrays. The strategic question that surfaces is: How large should the page size really be? In CD or DVD systems, the detected signal is from $50 \%$ to $20 \%$ of the incident power, while in holographic storage this usually does not exceed the range of $0.1 \%$ to $1 \%$. On the other hand, single bits are recorded in DVD systems having projected data rates of a few hundred megabits per second; not too far from the 500 $\mathrm{Mb} / \mathrm{s}$ required for follow-on systems. There is, therefore, an opportunity to use holography with very small page sizes (or even single-bit holograms), as has been researched by Eichler in Germany and Siros Technologies in the United States, among others [141], [142], and earlier at IBM. [143]. Small page sizes have significant advantages. The SLM and detector arrays can be made cheap and small, but they require very fast switching times, approaching a few nanoseconds. Wavelength multiplexing and or combined with spatial multiplexing of a few holograms would be sufficient to achieve data rates exceeding 1 $\mathrm{Gb} / \mathrm{s}$ and densities commensurate with capacities of $>500$ GB on a $120-$ mm disk.

Small page size simplifies and makes cheaper the optical design of high NA imaging optics, where a high NA is critical for achieving high density per hologram. The latter is important, since it results in higher diffraction efficiency of recorded holograms due to their reduced number (perhaps as few as 10-50) per multiplexed location. Higher bit density per hologram means that fewer holograms have to be superimposed, which improves diffraction efficiency and SNR. The high NA approach was successfully exploited in the Stanford/HDSS system and most later designed demonstration platforms described above. For small page sizes, Bragg degeneracy is less of an issue and tolerances are increased, improving manufacturability. Additionally, a small page size holographic system would afford a smoother transition from the single-bit DVD system to a holographic optical storage system. On the other hand, associative search would lose its attractiveness, as the number of pixels under the head will be small; therefore, such a holographic system would not have the advantage of fast search capabilities 


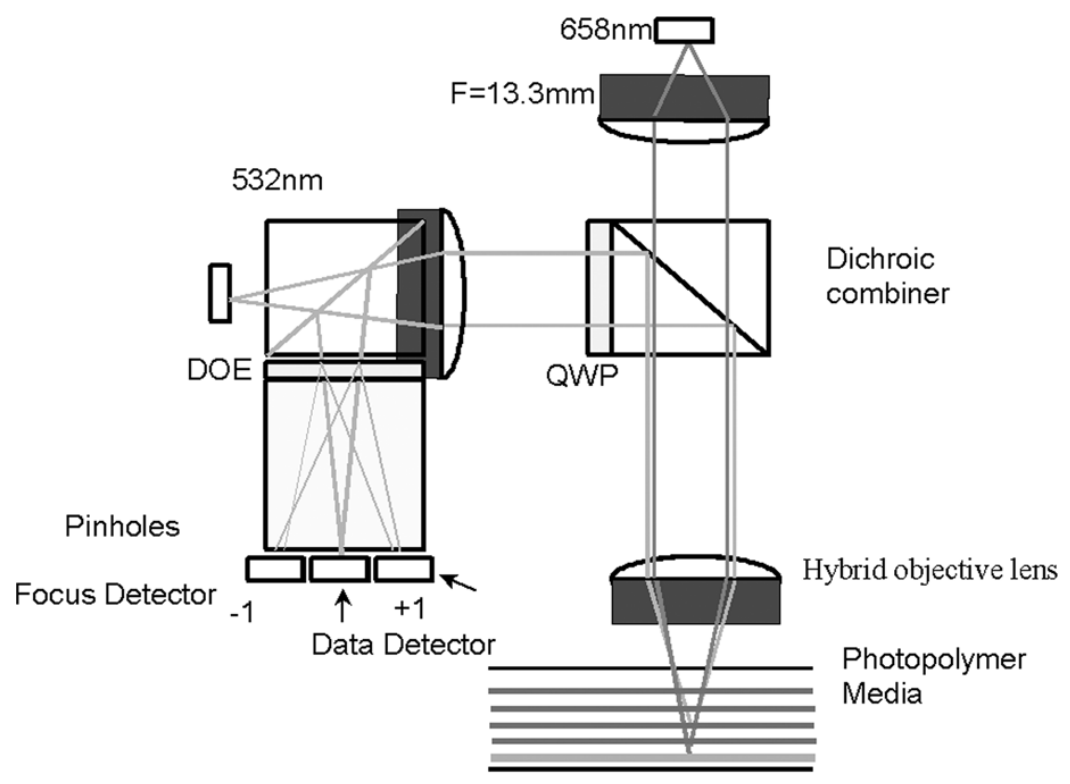

Fig. 80. Dual laser system for recording and readout in green and servoing in red. The single-servo layer is located below the virtual holographic data layers (courtesy Siros, with permission).

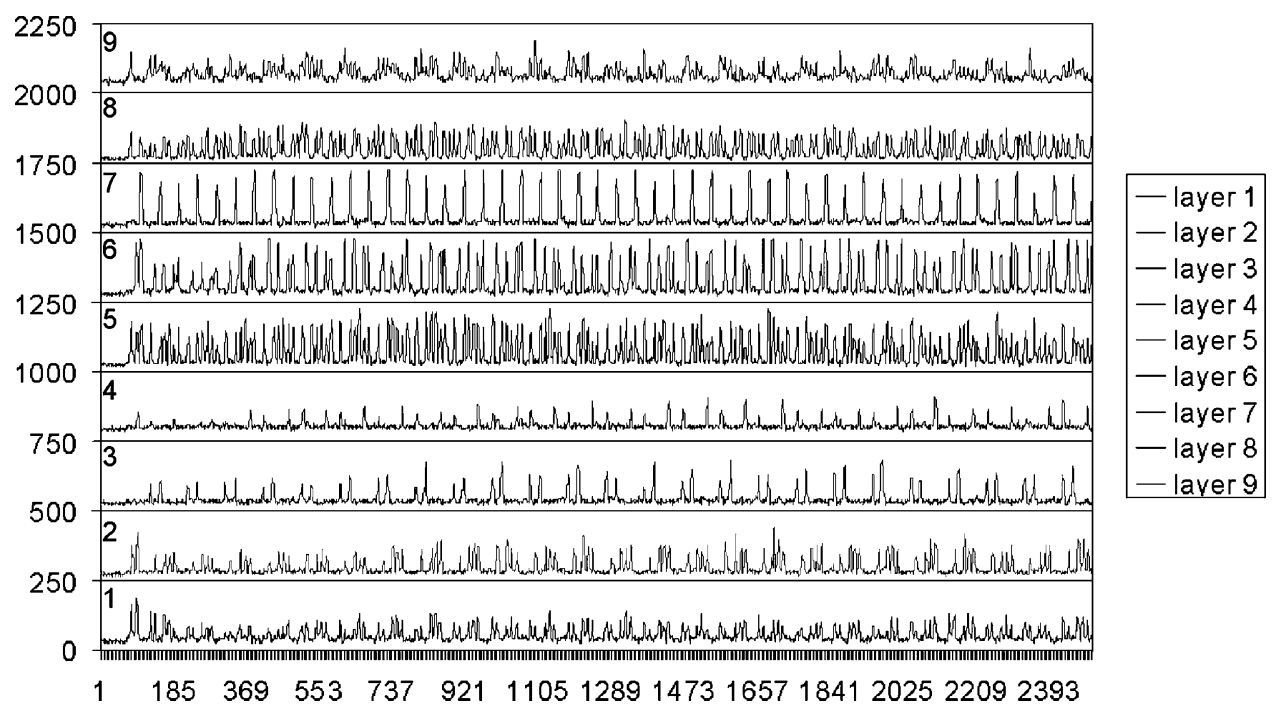

Fig. 81. Experimental results for reading nine layers of holographic single bit data recorded in Aprilis CROP photopolymer media using an NA 0.5 lens and 532-nm laser light (courtesy Siros, with permission).

over other forms of single bit storage, such as magnetic or solid-state memories. Finally, paged based systems require massively parallel data processing for ECC and channel coding, potentially adding significantly to the cost of holographic systems. Encoding and decoding chips for Reed-Solomon and, more recently, turbocodes are commercially available and can be used in parallel to achieve the desired data transfer rates. Smaller page sizes require less image processing steps and, therefore, encoding and decoding of data is simplified.

In short, when the page size becomes smaller, there is a higher tolerance to shrinkage, the tolerances for media flatness and wedge are greatly reduced, the tolerances on high NA imaging optics and the cost are reduced, and simpler electronic control systems can be utilized for servoing and tracking, as well as smaller lasers. These benefits are at the expense of reduced data transfer rate. Single-bit holograms provide an evolutionary path to improvement in the performance of optical data storage systems, versus a revolutionary (and much more challenging) improvement of larger size holographic page based storage systems. One distinct advantage of the latter approach is the enormously high-performance associative search capabilities which are not feasible with holographic systems having small page sizes.

Finally, the issue of replication needs to be considered. The great commercial success of CD-ROM and DVD-ROM technology has been partially due to the low cost of replication. CD- and DVD-ROM and recordable disks are stamped out at a rate of one disk every few seconds at a cost that is so low that customers use them as disposable media-very 


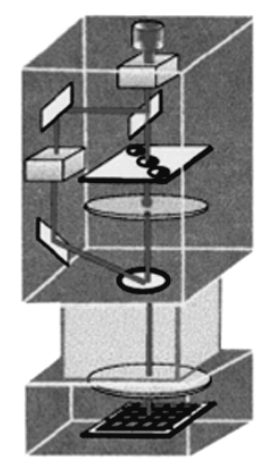

Extended DRAM product

- Total capacity: $25 \mathrm{~GB}$

- Access time: 10 ns

- Nonvolatile cache

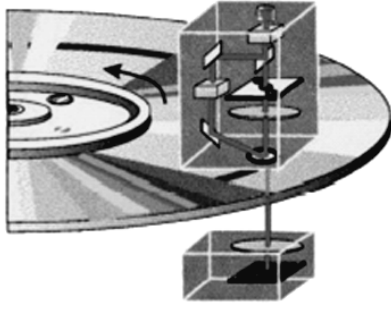

Hard disk drive (DASD) product

- High capacity: $1 \mathrm{~TB}$

- Access time: $10 \mathrm{~ms}$

- High reliability/availability

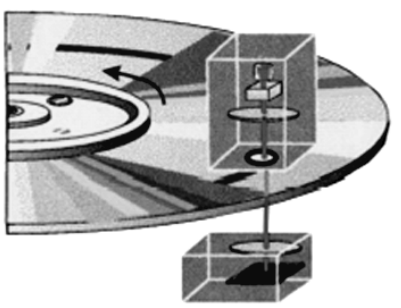

Hard disk drive (CDROM-type) product

- High capacity: 1 TB

- Access time: $10 \mathrm{~ms}$

- High capacity/low cost

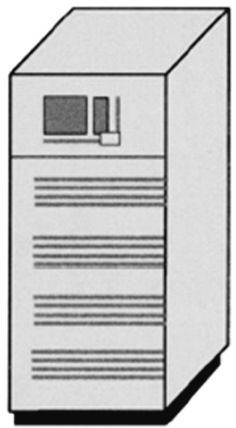

Rack-mounted archive product

- High capacity: 1 PB

- Access time: $10 \mathrm{~s}$

- High volumetric density

Fig. 82. Four possible holographic storage commercialization scenarios: an all-solid-state memory module having extremely short access times, two rotating disk geometries, with either erasable or WORM type media, and a data warehouse with removable media. With its high volumetric density, holographic storage has the potential to affect all types of data storage (courtesy IBM, with permission).

much the same way as previous floppy disks were used, but with 10000 times improved capacity. The removability and interchangeability of DVD media is a key to its success and these attributes need to be preserved in future generation optical removable storage technology. Although page-based holographic data storage technology provides far-field optical recording and readout, unfortunately it does not lend itself to simple copying procedures. As a result of multiplexing many holograms in the same location through either wavelength or angle multiplexing, a single-step replication process is not practical, as all reference beam angles and wavelengths must be simultaneously present to record data on the copied disk. So far, no good solution has been found for this problem [144], and holographic system demonstrations to date have been all of the WORM or rewritable kind. Single-bit virtual multilayer holographic recording may have an advantage in this respect over large page based holographic recording, as replication techniques can be envisioned. The single bits are recorded in the counterpropagating reflection geometry and a single, properly designed reference beam can be used to create a copy of a master hologram in a manner similar to techniques used for making copies of display holograms [145].

\section{SUMMARY}

Research on HDSSs has progressed significantly since the mid-1990s. New organic and inorganic materials have been developed with much improved properties. $\mathrm{LiNbO}_{3}$ is still the best quality optical medium for data storage as it does not shrink. Today, the best results in terms of density have been demonstrated in this medium. Recording in $\mathrm{LiNbO}_{3}$, however, is slow, and it is difficult to store large amounts of data due to size limitations. A jukebox approach could overcome this problem, but access times would be long, hundreds of milliseconds at best. For a single crystal, however, access times to any stored data can be on the order of mi- croseconds, and the $90^{\circ}$ configuration is ideally suited for associative retrieval at rates in excess of tens to hundreds of gigabits per second, and for direct data readout rates exceeding $1 \mathrm{~Gb} / \mathrm{s}$. Recording speeds are typically two orders of magnitude slower. Fixing of information requires elevated temperatures, but this might be acceptable in server applications, for example, where data are recorded occasionally, with readout by multiple users being the dominant use of the stored data. Cost of the medium could be brought down to $\$ 10-\$ 20$ per cubic centimeter $(\sim 10 \mathrm{~GB})$, produced in large volume. $\mathrm{LiNbO}_{3}$, therefore, is still a very attractive medium for holographic data storage for certain niche applications, such as for extremely rapid searches and in certain airborne and hostile environments where all-solid-state operation is required.

A jukebox approach, however, is not attractive as replacement for DVD technology. A rotating disk architecture provides shorter access times than a jukebox and "looks and feels" like a conventional disk drive. A significant effort has, therefore, been expended to realize such a system with superior performance over current optical drives. The HDSS demonstration, with $10 \mathrm{~Gb} / \mathrm{s}$ as the highest data transfer rate of any storage system, was made with this approach. The density achieved in this demonstration, however, was still too low. Due to material shrinkage and relatively high scatter levels, and limitations in shift multiplexing, the maximum density achieved so far (and published for verification) is 20 $\mathrm{b} / \mu \mathrm{m}^{2}$. This is about an order of magnitude too low for commercial viability of holographic technology. Unfortunately, for holographic data storage, density and transfer rate require a tradeoff of one against the other. As such, achieving either high density or transfer rate in isolation is not sufficient, as both need to be demonstrated in the same experimental setup. The challenge for holographic data storage technology to be a viable commercial product, as we see it, is to achieve a capacity of exceeding $200 \mathrm{~GB}$ on a 5.25-in disk, with a transfer 
rate in excess of $500 \mathrm{Mb} / \mathrm{s}$. The best photopolymer medium we have tested is made by Aprilis. With this medium, data densities corresponding to $250 \mathrm{~GB}$ on a DVD disk have been demonstrated. The challenge is now to combine the high data storage capacity and the ultrahigh transfer rate in a single testbed demonstration having a capacity of $250 \mathrm{~GB}$ and a transfer rate exceeding $500 \mathrm{Mb} / \mathrm{s}$. The outlook for such a demonstration is very good and can reasonably be expected within the next few years. Achieving that goal would go a long way toward establishing holographic data storage as a viable follow-on technology to current DVD systems.

\section{ACKNOWLEDGMENT}

The authors would like to thank Dr. L. N. Durvasula of DARPA for his pioneering view of HDSS and for his support of the NSIC/DARPA/Industry HDSS/PRISM consortia. Without his support, many of the improvements in holographic data storage would not have happened. The authors would also like to thank all the members of the two DARPA consortia for their contributions to the HDSS demonstration. The authors would also like to thank the Japanese Science and Technology Program.

\section{REFERENCES}

[1] J. Ashley, M.-P. Bernal, G. W. Burr, H. Coufal, H. Guenther, J. A. Hoffnagle, C. M. Jefferson, B. Marcus, R. M. Macfarlane, and R. M. Shelby, "Holographic data storage," IBM J. Res. Develop., vol. 44, pp. 341-368, 2000.

[2] L. Hesselink and M. C. Bashaw, "Optical memories implemented with photorefractive media," Opt. Quantum Electron., vol. 25, pp. S611-S661, 1993.

[3] A. J. Daiber, R. Snyder, J. Colvin, R. Okas, and L. Hesselink, "Fully functional digital video holographic storage system," presented at the Optical Soc. Amer. Annu. Meeting, Long Beach, CA, 1997, Paper ThR3.

[4] R. M. Shelby, J. A. Hoffnagle, G. W. Burr, C. M. Jefferson, M.-P. Bernal, H. Coufal, R. K. Grygier, H. Gunther, R. M. Macfarlane, and G. T. Sincerbox, "Pixel-matched holographic data storage with megabit pages," Opt. Lett., vol. 22, pp. 1509-1511, 1997.

[5] J. H. Hong, I. McMichael, T. Y. Chang, W. Christian, and E. G. Paek, "Volume holographic memory systems: Techniques and architectures," Opt. Eng., vol. 34, pp. 2193-2203, 1995.

[6] D. Psaltis and G. W. Burr, "Volume holographic memory systems: Techniques and architectures," Computer, vol. 31, pp. 52-60, 1998.

[7] K. Curtis, "Digital holographic data storage prototype," in Conf. Rec. 2000 Optical Data Storage, pp. 164-166.

[8] F. H. Mok, "Angle-multiplexed storage of 5000 holograms in lithium niobate," Opt. Lett., vol. 18, pp. 915-917, 1993.

[9] G. Barbastathis and D. Psaltis, "Volume holographic multiplexing methods," in Holographic Data Storage, H. Coufal, D. Psaltis, and G. T. Sincerbox, Eds. Berlin, Germany: Springer-Verlag, 2000, pp. 21-62.

[10] L. Hesselink, "Digital holographic demonstration systems by Stanford University and Sires Technologies," in Holographic Data Storage, H. Coufal, D. Psaltis, and G. T. Sincerbox, Eds. Berlin, Germany: Springer-Verlag, 2000, pp. 383-397.

[11] D. Psaltis, M. Levene, A. Pu, G. Barbastathis, and K. Curtis, "Holographic storage using shift multiplexing," Opt. Lett., vol. 20, pp. 782-784, 1995.

[12] A. M. Darskii and V. B. Markov, "Shift selectivity of holograms with a reference speckle wave," Opt. Spectmsc. (USSR), vol. 65, pp. 392-395, 1988

[13] T. Bieringer, "Photoaddressable polymers," in Holographic Data Storage, H. Coufal, G. T. Sincerbox, and D. Psaltis, Eds. Berlin, Germany: Springer-Verlag, 2000, pp. 209-230.
[14] S. S. Orlov, E. Bjornson, W. Phillips, Y. Takashima, X. Lee, L. Hesselink, R. Okas, and R. Snyder, "High transfer rate $(1 \mathrm{Gbit} / \mathrm{sec})$ high capacity holographic disk digital data storage system," in Proc. Conf. Lasers and Electro-Optics (CLEO 2000), pp. 190-191.

[15] D. A. Waldman, H.-Y. S. Li, and M. G. Horner, "Volume shrinkage in slant fringe gratings of a cationic ring-opening volume hologram recording material," J. Imag. Sci. Technol., vol. 41, pp. 497-514, 1997.

[16] L. Dhar, K. Curtis, M. Tackitt, M. Schilling, S. Campbell, W. Wilson, A. Hill, C. Boyd, N. Levinos, and A. Harris, "Holographic storage of multiple high-capacity digital data pages in thick photopolymer systems," Opt. Lett., vol. 23, pp. 1710-1722, 1998.

[17] S. S. Orlov, W. Phillips, E. Bjornson, L. Hesselink, and R. Okas, "10 Gigabit/second sustained optical data transfer rate from a holographic disk digital data storage system," presented at the OSA Annual Meeting 2000, Paper MK3.

[18] S. S. Orlov, "Volume holographic data storage," Commun. ACM, vol. 43, pp. 46-55, 2000.

[19] L. d'Auria, J.-P. Huignard, C. Slezak, and E. Spitz, "Experimental holographic read-write memory using 3-D storage," Appl. Opt., vol. 13, pp. 808-818, 1974.

[20] J. B. Thaxter and M. Kestigian, "Unique properties of SBN and their use in a layered optical memory," Appl. Opt., vol. 13, pp. 913-924, 1974.

[21] A. Mikaeliane et al., "Holographic bulk memories using lithium niobate crystals for data recording," in Optical Information Recording, E. S. Barrekette et al., Eds. New York: Plenum, 1978, vol. 2, pp. 217-233.

[22] P. J. van Heerden, "Theory of optical information storage in solids," Appl. Opt., vol. 2, pp. 393-400, 1963.

[23] S. Redfield, "Tamarack optical head holographic storage," in Holographic Data Storage, H. Coufal, G. T. Sincerbox, and D. Psaltis, Eds. Berlin, Germany: Springer-Verlag, 2000, pp. 343-357.

[24] J. F. Heanue, M. C. Bashaw, and L. Hesselink, "Volume holographic storage and retrieval of digital data," Science, vol. 265, pp. 749-752, 1994.

[25] D. Psaltis, D. Brady, and K. Wagner, "Adaptive optical networks using photorefractive crystals," Appl. Opt., vol. 27, pp. 1752-1759, 1988.

[26] P. Yeh, Introduction to Photorefractive Nonlinear Optics. New York: Wiley, 1993.

[27] M. C. Bashaw, J. F. Heanue, and L. Hesselink, "Organization of data for monochromatic multiplex volume holography," J. Opt. Soc. Amer. A, vol. 13, pp. 2174-2186, 1996.

[28] C. X.-G. Gu, "Optical neural networks using volume holography," $\mathrm{PhD}$ dissertation, California Inst. Technol., Pasadena, 1990.

[29] M. A. Neifeld and W. C. Chou, "Information theoretic limits to the capacity of volume holographic optical memory," Appl. Opt., vol. 36, pp. 514-517, 1997.

[30] G. W. Burr, C. M. Jefferson, H. Coufal, M. Jurich, T. A. Hoffnagle, R. M. Macfarlane, and R. M. Shelby, "Volume holographic data storage at an areal density of 250 gigapixels/in2," Opt. Lett., vol. 26, pp. 444-446, 2001.

[31] C. Gu, J. Hong, I. McMichael, R. Saxena, and F. Mok, "Cross-talklimited storage capacity of volume holographic memory," J. Opt. Soc. Amer. A, vol. 9, pp. 1978-1983, 1992.

[32] M. C. Bashaw, J. F. Heanue, A. Aharoni, J. F. Walkup, and L. Hesselink, "Crosstalk considerations for angular and phase-encoded multiplexing in volume holography," J. Opt. Soc. Amer. B, vol. 11, pp. 1820-1836, 1994.

[33] E. N. Leith, A. Kozma, J. Upatnieks, J. Marks, and N. Massey, "Holographic data storage in three-dimensional media," Appl. Opt., vol. 5, pp. 1303-1311, 1966.

[34] A. Yariv, G. Rakuljic, A. Yariv, and V. Leyva, "High resolution volume holography using orthogonal data storage," presented at the Tech. Dig. Photorefractive Materials, Effects, and Devices 1991, Paper MD-3.

[35] X. Yi, P. Yeh, C. Gu, and S. Campbell, "Crosstalk in volume holographic memory," Proc. IEEE, vol. 87, pp. 1912-1930, Nov. 1999.

[36] C. Gu, P. Yeh, X. Yi, and J. Hong, "Fundamental noise sources in volume holographic storage," in Holographic Data Storage, H. Coufal, D. Psaltis, and G. T. Sincerbox, Eds. Berlin, Germany: Springer-Verlag, 2000, pp. 63-88.

[37] K. Curtis, C. Gu, and D. Psaltis, "Cross talk in wavelength-multiplexed holographic memories," Opt. Lett., vol. 18, pp. 1001-1003, 1993. 
[38] D. Lande, J. F. Heanue, M. C. Bashaw, and L. Hesselink, "Digital wavelength-multiplexed holographic data storage system," Opt. Lett., vol. 21, pp. 1780-1782, 1996.

[39] T. F. Krile, R. J. Marks II, J. F. Walkup, and M. O. Hagler, "Holographic representations of space-variant systems using phase-coded reference beams," Appl Opt., vol. 16, pp. 3131-3135, 1977.

[40] T. F. Krile, M. O. Hagler, W. D. Redus, and J. F. Walkup, "Multiplex holography with chirp-modulated binary phase-coded reference-beam masks," Appl. Opt., vol. 18, pp. 52-56, 1979.

[41] V. N. Morozov, "Theory of holograms formed using coded reference beams," Kvantovaya Elektron., vol. 4, pp. 1694-1701, 1977. English transl.: Sov. J. Quantum. Electron., vol. 7, pp. 961-964, 1977.

[42] D. Gabor, "Associative holographic memories," IBM J. Res. Develop., pp. 156-159, Mar. 1969.

[43] E. L. Kral, J. F. Walkup, and M. O. Hagler, "Correlation properties of random phase diffusers for multiplex holography," Appl. Opt., vol. 21, pp. 1281-1290, 1982

[44] A. E. Krasnov, "Thick-film phase holograms recorded by means of coded reference waves," Kvantovaya Elektron., vol. 4, pp. 2011-2013, 1977. English transl.: Sov. J. Quantum. Electron., vol. 7, pp. 1147-1148, 1977.

[45] C. Denz, K. O. Miller, F. Visinka, and T. Tschudi, "Demonstration platform for phase-coded multiplexing," in Holographic Data Storage, H. Coufal, D. Psaltis, and G. T. Sincerbox, Eds. Berlin, Germany: Springer-Verlag, 2000, pp. 419-428.

[46] G. Barbastathis, M. Levene, and D. Psaltis, "Shift multiplexing with spherical reference waves," Appl. Opt., vol. 35, pp. 2403-2417, 1996.

[47] L. Hesselink, "Photorefractive fibers for optical data storage and processing," Intl. J. Optoelectron., vol. 5, pp. 103-124, 1990.

[48] S. Wu, A. Mayers, S. Rajan, and F. T. S. Yu, "Use of photorefractive fiber in optical interconnections and switching," Appl. Opt., vol. 29, pp. 1059-1061, 1990.

[49] F. Ito, K.-I. Kitayama, and H. Oguri, "Compensation of fiber holographic image distortion caused by intrasignal photorefractive coupling by using a phase-conjugated mirror," Opt. Lett., vol. 17, pp. 215-217, 1992.

[50] M. C. Bashaw, A. Aharoni, and L. Hesselink, "Alleviation of image distortion due to striations in a photorefractive medium by using a phase-conjugated reference wave," Opt. Lett., vol. 17, pp. 1149-1151, 1992.

[51] A. Aharoni, M. C. Bashaw, and L. Hesselink, "Distortion-free multiplexed holography in striated photorefractive media," Appl. Opt., vol. 32, pp. 1973-1982, 1993.

[52] E. Chuang, W. Liu, J. P. Drolet, and D. Psaltis, "Holographic random access memory (HRAM)," Proc. IEEE, vol. 87, pp. 1931-1940, Nov. 1999.

[53] G. W. Burr and I. Leyva, "Multiplexed phase-conjugate holographic data storage with a buffer hole gram," Opt. Lett., vol. 25, pp. 499-501, 2000.

[54] G. W. Burr, S. Kobras, H. Hanssen, and H. Coufal, "Content-addressable data storage using volume holograms," Appl. Opt., vol. 38, pp. 6779-6784, 1999.

[55] X. Li, F. Dimov, W. Phillips, L. Hesselink, and R. McLeod, "Parallel associate search by use of volume holographic memory," in Proc. 29th Applied Imagery and Pattern Recognition Workshop (AIPR 2000), J. Aanstoos, Ed., pp. 78-83.

[56] S. S. Orlov, "Overview of holographic recording materials for major system architectures in holographic data storage applications," presented at the National Storage Industry Consortium Int. Workshop Holographic Data Storage, Nice, France, 1999.

[57] M.-P. Bernal, H. Coufal, R. K. Grygier, J. A. Hoffnagle, C. M. Jefferson, R. M. Macfarlane, R. M. Shelby, G. T. Sincerbox, P. Wimmer, and G. Wittmann, "A precision tester for studies of holographic optical storage materials and recording physics," Appl. Opt., vol. 35, pp. $2360-2374,1996$.

[58] R. M. Shelby, "Media requirements for digital holographic data storage," in Holographic Data Storage, H. Coufal, D. Psaltis, and G. T. Sincerbox, Eds. Berlin, Germany: Springer-Verlag, 2000, pp. 101-112.

[59] P. Günter and J.-P. Huignard, "Photorefractive effects and materials," in Photorefractive Materials and Their Applications I, P. Günter and J.-P. Huignard, Eds. Berlin, Germany: Springer-Verlag, 1988, pp. $7-73$.

[60] H. W. Kogelnik, "Coupled wave theory for thick hologram gratings," Bell Syst. Tech. J., vol. 48, pp. 2909-2947, 1969.
[61] V. L. Vinetskii and N. V. Kukhtarev, "Theory of the conductivity induced by recording holographic gratings in nonmetallic crystals," Fiz. Tverd. Tela, vol. 16, pp. 3714-3716, 1974. English transl.: Sov. Phys. Solid State, vol. 16, p. 2414, 1975.

[62] N. V. Kukhtarev, "Kinetics of hologram recording and erasure in electrooptic crystals," Pis'ma Zh. Tekh. Fiz., vol. 2, pp. 1114-1119, 1976. English transl.: Sov. Tech. Phys. Lett., vol. 2, p. 438, 1976.

[63] N. V. Kukhtarev, V. B. Markov, S. G. Odulov, M. S. Soskin, and V. L. Vinetskii, "Holographic storage in electrooptic crystals. I. Steady state," Ferroelectrics, vol. 22, pp. 949-960, 1979.

[64] G. C. Valley, "Erase rates in photorefractive materials with two photoactive species," Appl. Opt., vol. 22, pp. 3160-3164, 1983.

[65] G. C. Valley and J. F. Lam, "Theory of photorefractive effects in electro-optic crystals," in Photorefractive Materials and Their Applications I, P. Günter and J.-P. Huignard, Eds. Berlin, Germany: Springer-Verlag, 1988, pp. 75-98.

[66] A. Yariv, S. S. Orlov, and G. A. Rakuljic, "Holographic storage dynamics in lithium niobate: theory and experiment," J. Opt. Soc. Amer. B, vol. 13, pp. 2513-2523, 1996.

[67] B. Kippelen, "Overview of photorefractive polymers for holographic data storage," in Holographic Data Storage, H. Coufal, D. Psaltis, and G. T. Sincerbox, Eds. Berlin, Germany: Springer-Verlag, 2000, pp. 159-169.

[68] D. L. Staebler, W. J. Burke, W. Phillips, and J. J. Amodei, "Multiple storage and erasure of fixed holograms in Fe-doped $\mathrm{LiNbO}_{3}$," Appl. Phys. Lett., vol. 26, pp. 182-184, 1975.

[69] M. Carrascosa and F. Agulló-Löpez, "Theoretical modeling of the fixing and developing of holographic gratings in $\mathrm{LiNbO}_{3}, " \mathrm{~J}$. Opt. Soc. Amer. B, vol. 7, pp. 2317-2322, 1990.

[70] G. Montemezzani, M. Zgonik, and P. Günter, "Photorefractive charge compensation at elevated temperatures and application to $\mathrm{KNbO}_{3}$, J. Opt. Soc. Amer. B, vol. 10, pp. 171-185, 1993.

[71] A. Yariv, S. Orlov, G. Rakuljic, and V. Leyva, "Hologram fixing, readout, and storage dynamics in photorefractive materials," Opt. Lett., vol. 20, pp. 1334-1336, 1995.

[72] J. J. Amodei and D. L. Staebler, "Holographic pattern fixing in electro-optic crystals," Appl. Phys. Lett., vol. 18, pp. 540-542, 1971.

[73] S. S. Orlov and W. Phillips, "Hologram fixing and nonvolatile storage in photorefractive materials," in Holographic Data Storage, H. Coufal, D. Psaltis, and G. T. Sincerbox, Eds. Berlin, Germany: Springer-Verlag, 2000, pp. 127-148.

[74] H. Kurz, "Lithium niobate as a material for holographic information storage," Philips Tech. Rev., vol. 37, pp. 109-120, 1977.

[75] _ - "Photorefractive recording dyanmics and multiple storage of volume holograms in photorefractive $\mathrm{LiNbO}_{3}$, , Optica Acta, vol. 24, pp. 463-473, 1977.

[76] W. J. Burke, D. L. Staebler, W. Phillips, and G. A. Alphonse, "Volume phase holographic storage in ferroelectric crystals," Opt. Eng., vol. 17, pp. 308-316, 1978.

[77] J. F. Heanue, M. C. Bashaw, A. J. Daiber, R. Snyder, and L. Hesselink, "Thermal fixing for digital holographic data storage," Opt. Lett., vol. 21, pp. 1615-1617, 1996.

[78] X. An, D. Psaltis, and G. W. Burr, "Thermal fixing of 10000 holograms in $\mathrm{LiNbO}_{3}$, , Appl. Opt., vol. 38, pp. 386-393, 1999.

[79] D. von der Linde, A. M. Glass, and K. F. Rodgers, "High-sensitivity optical recording in KTN by two-photon absorption," Appl. Phys. Lett., vol. 26, pp. 22-24, 1975.

[80] —, "Optical storage using refractive index changes induced by two-step excitation,” J. Appl. Phys., vol. 47, pp. 217-220, 1976.

[81] J. P. Wilde, "Spectroscopic characterization of photorefractive materials for holographic storage applications," in Proc. SPIE, Fluorescence Detection Conf., 1996, pp. 82-92.

[82] Y. S. Bai, R. R. Neurgaonkar, and R. Kachru, "Resonant two-photon photorefractive gating in praeseodymium-doped strontium barium niobate with cw lasers," Opt. Lett., vol. 21, pp. 567-569, 1996.

[83] S. S. Orlov, A. Akella, L. Hesselink, and R. R. Neurgaonkar, "High sensitivity two-photon nonvolatile recording in lithium niobate," presented at the Conf. Lasers and Electro-Optics (CLEO), 1997, Postdeadline Paper CPD29.

[84] L. Hesselink, S. S. Orlov, A. Liu, A. Akella, D. Lande, and R. R. Neurgaonkar, "Photorefractive materials for nonvolatile volume holographic data storage," Science, vol. 282, pp. 1089-1094, 1998.

[85] D. von der Linde, A. M. Glass, and K. F. Rodgers, "Multiphoton photorefractive processes for optical storage in $\mathrm{LiNbO}_{3}$," Appl. Phys. Lett., vol. 25, pp. 155-157, 1974. 
[86] D. von der Linde and A. M. Glass, "Photorefractive effects for reversible holographic storage of information," Appl. Phys., vol. 85, pp. 85-100, 1975

[87] H. Vormann and E. Krätzig, "Two step excitation in $\mathrm{LiTaO}_{3}$ : Fe for optical data storage," Solid State Commun., vol. 49, pp. 843-847, 1984.

[88] D. L. Staebler and W. Phillips, "Hologram storage in photochromic $\mathrm{LiNbO}_{3}$," Appl. Phys. Lett., vol. 24, pp. 268-270, 1974.

[89] K. Buse, A. Adibi, and D. Psaltis, "Non-volatile holographic storage in doubly doped lithium niobate crystals," Nature, vol. 393, pp. 665-668, 1998.

[90] D. Lande, S. S. Orlov, A. Akella, D. Lande, and R. R. Neurgaonkar, "Digital holographic storage system incorporating optical fixing," Opt. Lett., vol. 22, pp. 1722-1724, 1997.

[91] D. Tontchev, S. Zhivkova, and M. Miteva, "Holographic interferometric microscope on the basis of a $\mathrm{Bi}_{12} \mathrm{TiO}_{20}$ crystal," Appl. Opt., vol. 29, pp. 4753-4756, 1990.

[92] S. Zhivkova and M. Miteva, "Image subtraction using fixed holograms in photorefractive $\mathrm{Bi}_{12} \mathrm{SiO}_{20}$ crystals," Opt. Lett., vol. 16, pp. 750-751, 1991.

[93] M. C. Bashaw, T.-P. Ma, R. C. Barker, S. Mroczkowski, and R. R. Dube, "Introduction, revelation, and evolution of complementary holograms in photorefractive bismuth silicon oxide," Phys. Rev. B, vol. 42, pp. 5641-5648, 1990.

[94] S. Zhivkova and M. Miteva, "Investigations of the characteristics of fixed holograms in $\mathrm{Bi}_{12} \mathrm{TiO}_{20}$ photorefractive crystals," Opt. Commun., vol. 86, pp. 449-453, 1991.

[95] N. A. Vainos, S. L. Clapham, and R. W. Eason, "Multiplexed permanent and real time holographic recording in photorefractive BSO," Appl. Opt., vol. 28, pp. 4381-4385, 1989.

[96] - "Applications of multiplexed real time and permanent holographic recording in photorefractive BSO," Appl. Opt., vol. 28, pp. 4386-4392, 1989.

[97] V. Leyva, A. Agranat, and A. Yariv, "Fixing of a photorefractive grating in $\mathrm{KTa}_{1-x} \mathrm{Nb}_{\mathrm{X}} \mathrm{O}_{3}$ by cooling through the ferroelectric phase transition," Opt. Lett., vol. 16, pp. 554-556, 1991.

[98] F. Micheron and G. Bismuth, "Field and time thresholds for the electrical fixation of holograms recorded in $\left(\mathrm{Sr}_{0.75} \mathrm{Ba}_{0.25}\right) \mathrm{Nb}_{2} \mathrm{O}_{6}$ crystals," Appl. Phys. Lett., vol. 23, pp. 71-72, 1973.

[99] - "Electrical control of fixation and erasure of holographic patterns in ferroelectric materials," Appl. Phys. Lett., vol. 20, pp. 79-81, 1972.

[100] S. Orlov, D. Psaltis, and R. R. Neurgaonkar, "Dynamic electronic compensation of fixed gratings in photorefractive media," Appl. Phys. Lett., vol. 63, pp. 2466-2468, 1993.

[101] J. Ma, T. Chang, J. Hong, R. Neurgaonkar, G. Barbastathis, and D. Psaltis, "Electrical fixing of 1000 angle-multiplexed holograms in SBN:75," Opt. Lett., vol. 22, pp. 1114-1116, 1997.

[102] E. K. Gulanyan, I. R. Dorosh, V. D. Iskin, A. L. Mikaelyan, and M. A. Maiorchuk, "Nondestructive readout of holograms in irondoped lithium niobate crystals," Kvantovaya Elektron., vol. 6, pp. 1100-2097, 1979. English transl.: Sov. J. Quantum Electron., vol. 9 , pp. $647-649,1979$.

[103] H.-C. Külich, "Transfer function for image formation of objects reconstructed from volume holograms with different wavelengths," Appl. Opt., vol. 31, pp. 2461-2477, 1992.

[104] D. Psaltis, F. H. Mok, and H.-Y. S. Li, "Nonvolatile storage in photorefractive crystals," Opt. Lett., vol. 19, pp. 210-212, 1994

[105] A. Aharoni, M. Jeganathan, M. C. Bashaw, and L. Hesselink, "Prolonged readout of photorefractive holograms by replay at a longer wavelength," presented at the Conf. Lasers and Electro-Optics, 1994, Paper CTuJ4.

[106] D. Brady, K. Hsu, and D. Psaltis, "Periodically refreshed multiply exposed photorefractive holograms," Opt. Lett., vol. 15, pp. 817-819, 1990

[107] H. Sasaki, Y. Fainman, J. E. Ford, Y. Taketomi, and S. H. Lee, "Dynamic photorefractive optical memory," Opt. Lett., vol. 16, pp. 1874-1876, 1991

[108] Y. Qiao, D. Psaltis, C. Gu, J. Hong, P. Yeh, and R. R. Neurgaonkar, "Phase-locked sustainment of photorefractive holograms using phase conjugation," J. Appl. Phys., vol. 70, pp. 4646-4648, 1991.

[109] D. Brady and D. Psaltis, "Control of volume holograms," J. Opt. Soc. Amer. A, vol. 9, pp. 1167-182, 1992.

[110] S. Boj, G. Pauliat, and G. Roosen, "Dynamic holographic memory showing readout, refreshing, and updating capabilities," Opt. Lett., vol. 17, pp. 438-440, 1992.
[111] Y. Qiao and D. Psaltis, "Sampled dynamic holographic memory," Opt. Lett., vol. 17, pp. 1376-1378, 1992.

[112] H. Rajbenbach, S. Bann, and J.-P. Huignard, "Long-term readout of photorefractive memories by using a storage/amplification twocrystal configuration," Opt. Lett., vol. 17, pp. 1712-1714, 1992.

[113] S. Odoulov, T. Tarabrova, A. Shumelyuk, L. I. Naumova, and T. O. Chaplina, "Photorefractive response of bulk periodically poled $\mathrm{LiNbO}_{3}: \mathrm{Y}: \mathrm{Fe}$ at high and low spatial frequencies," Phys. Rev. Lett., vol. 84, pp. 3294-3297, 2000.

[114] R. De Vré, J. F. Heanue, K. Gürkan, and L. Hesselink, "Transfer functions based on Bragg detuning effects for image-bearing holograms recorded in photorefractive crystals," J. Opt. Soc. Amer. A, vol. 13, pp. 1331-1344, 1996.

[115] L. Dhar, M. G. Schnoes, H. E. Katz, A. Hale, M. L. Schilling, and A. L. Harris, "Photopolymers for digital holographic data storage," in Holographic Data Storage, H. Coufal, D. Psaltis, and G. T. Sincerbox, Eds. Berlin, Germany: Springer-Verlag, 2000, pp. 199-208.

[116] K. Curtis, W. L. Wilson, M. C. Tackitt, A. J. Hill, and S. Campbell, "High-density, high-performance data storage via volume holography: the Lucent Technologies hardware platform," in Holographic Data Storage, H. Coufal, D. Psaltis, and G. T. Sincerbox, Eds. Berlin, Germany: Springer-Verlag, 2000, pp. 359-368.

[117] A. Vander Lugt, "Design relationships for holographic memories," Appl. Opt., vol. 12, pp. 1675-1685, 1973.

[118] A. L. Mikaelian, V. L. Bobrinev, S. M. Naumova, and L. Z Sokolova, "Design principles of holographic memory devices," IEEE. J. Quantum Electron., vol. QE-6, pp. 193-198, Apr. 1970.

[119] W.-H. Lee, "Effect of film-grain noise on the performance of holographic memory," J. Opt. Soc. Am., vol. 62, pp. 797-801, 1972.

[120] J. W. Goodman, Statistical Optics. New York: Wiley, 1985.

[121] J. F. Heanue, M. C. Bashaw, and L. Hesselink, "Channel coding for digital holography," J. Opt. Soc. Amer. A, vol. 12, pp. 2432-2439, 1995.

[122] B. Marcus, "Modulation codes for holographic recording," in Holographic Data Storage, H. Coufal, D. Psaltis, and G. T. Sincerbox, Eds. Berlin, Germany: Springer-Verlag, 2000, pp. 283-292.

[123] G. W. Burr, J. Ashley, H. Coufal, R. Grygier, J. Hoffnagle, C. M. Jefferson, and B. Marcus, "Modulation coding for pixel-matched holographic data storage," Opt. Lett., vol. 22, pp. 639-641, 1997.

[124] R. Snyder and A. Daiber, "Method for holographic data storage and retrieval," U.S. Patent 6064 586, May 16, 2000.

[125] G. W. Burr and T. Weiss, "Compensation for pixel misregistration in volume holographic data storage," Opt. Lett., vol. 26, pp. 542-544, 2001.

[126] J. F. Heanue, K. Gurkan, and L. Hesselink, "Signal detection for page-access optical memories with intersymbol interference," Appl. Opt., vol. 35, pp. 2431-2438, 1996.

[127] V. Vadde and B. V. K. V. Kumar, "Channel modeling and estimation for intrapage equalization in pixel-matched volume holographic data storage," Appl. Opt., vol. 38, pp. 4374-4386, 1999.

[128] G. W. Burr, H. Coufal, R. K. Grygier, J. A. Hoffnagle, and C. M. Jefferson, "Noise reduction of page-oriented data storage by inverse filtering during recording," Opt. Lett., vol. 23, pp. 289-291, 1998.

[129] F. Mok, D. Psaltis, and G. Burr, "Spatially- and angle-multiplexed holographic random access memory," in Proc. SPIE, Photonic Neural Networks, vol. 1773, 1992, pp. 334-345.

[130] R. Brauer, U. Wojak, F. Wyrowski, and O. Bryngdahl, "Digital diffusers for optical holography," Opt. Lett., vol. 16, pp. 1427-1429, 1991.

[131] S. S. Orlov, R. Snyder, and M. C. Bashaw, "Optical relay for pixelbased holographic storage and retrieval,", Aug. 22, 2000.

[132] W. Phillips, S. S. Orlov, E. Bjornson, L. Hesselink, and R. Okas, "Video demonstration of high data rate holographic disk data storage system," presented at the OSA Annu. Meeting 2000, Paper MK2.

[133] C. M. Jefferson, G. W. Burr, and J. A. Hoffnagle, "IBM holographic data storage test platforms," in Holographic Data Storage, H. Coufal, D. Psaltis, and G. T. Sincerbox, Eds. Berlin, Germany: Springer-Verlag, 2000, pp. 369-381.

[134] J. A. Hollhagle and C. M. Jefferson, "Design and performance of a refractive optical system that converts a Gaussian to a flattop beam," Appl. Opt., vol. 39, pp. 5488-5499, 2000.

[135] J. Ma, T. Chang, S. Choi, and J. Hong, "Ruggedized digital holographic data storage with fast access," Opt. Quantum Electron., vol. 32 , pp. 383-392, 2000 
[136] F. Mok, G. Zhou, and D. Psaltis, "Holographic read-only memory," in Holographic Data Storage, H. Coufal, D. Psaltis, and G. T. Sincerbox, Eds. Berlin, Germany: Springer-Verlag, 2000, pp. 399-407.

[137] D. A. Waldman, C. I. Butler, and D. H. Raguin, "CROP holographic storage media for optical data storage at greater than $100 \mathrm{bits} / \mathrm{sq}$. micron," in Proc. SPIE, Organic Holographic Materials and Applications, vol. 5216, 2003, pp. 10-25.

[138] M. Schnoes, B. Ihas, A. Hill, L. Dhar, D. Michaels, S. Setthachayanon, G. Schomberger, and W. L. Wilson, "Holographic data storage media for practical systems," in Proc. SPIE, Practical Holography XVII and Holographic Materials IX, vol. 5005, 2003, pp. 29-37.

[139] S. Yagi, T. Imai, Y. Kurokawa, M. Endo, K. Ishihara, R. Yoshiyama, H. Kubo, and Y. Miwa, "Multilayered waveguide holographic ROM card," Trans. Inst. Electron. Inf. Commun. Eng. C, vol. J84-C, pp. 635-643, 2001.

[140] L. Hesselink, "Three dimensional recording (3DR) technology," in Tech. Dig. 2000 Optical Data Storage (ODS) Conf., pp. 149-151.

[141] H. J. Eichler, P. Kuemmel, S. Orloc, and A. Wappelt, "High-density disk storage by multiplexed microholograms," IEEE J. Select. Topics Quantum Electron., vol. 4, pp. 840-848, 1998.

[142] R. McLeod and L. Hesselink, Virtual Multilayer Holographic Data Storage, 2004, submitted for publication.

[143] A. W. Bidwell and G. T. Sincerbox, "Holographic storage system," IBM Tech. Disclosure Bull., vol. 12, pp. 1659-1660, 1970.

[144] F. Mok, G. Zhou, and D. Psaltis, "Holographic read-only memory," in Holographic Data Storage, H. Coufal, D. Psaltis, and G. T. Sincerbox, Eds. Berlin, Germany: Springer-Verlag, 2000, pp. 399-407.

[145] S. A. Benton, "Hologram reconstructions with extened incoherent sources," J. Opt. Soc. Amer., vol. 59, pp. 1545-1546A, 1969.

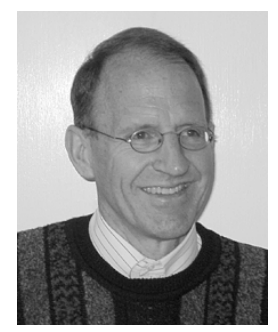

Lambertus Hesselink received the Ph.D. degree from the California Institute of Technology, Pasadena, in 1977.

He was the Principal Investigator of two large $\$ 52$ million Defense Advanced Research Projects Agency/industry/university consortia on the development of holographic data storage systems (HDSS) and photorefractive information storage media (PRISM) from 1995 to 2000. He founded Siros Technologies and Senvid, Inc., where he has held the positions of Chairman, Chief Executive Officer (CEO), and Chief Technology Officer (CTO). He was also a Visiting Professor in China, Europe, and Japan. He is currently Professor of Electrical Engineering and, by courtesy, Applied Physics at Stanford University, Palo Alto, CA. He is considered a worldwide leader in the development of ultrahigh-performance optical data storage systems and a pioneer in Internet-assisted learning. He has authored over 350 papers in archival journals and presented over 250 keynote and invited lectures at scientific meetings worldwide. He was Editor of Applied Optics and Applied Scientific Research, and he has over 50 patents and over 15 pending applications. His current research interests span the areas of ultrahigh density optical data storage and nanophotonics.

Dr. Hesselink is a Fellow of the Optical Society of America, a Fellow of the Society of Photo Instrumentation Engineers (SPIE), and a Member of the Royal Dutch Academy of Arts and Sciences. Among other honors, he was a Member of the Hubble Space Telescope Committee, a Member of the Scientific Advisory Board of the U.S. Air Force, a Codesigner of the official astronaut memorial at Space Port USA, a Fulbright Scholar, and a Stheeman Prize recipient. He has organized over 70 scientific meetings. He was an Editior of the IEEE TRANSACTIONS ON VISUALIZATION AND COMPUTER GRAPHICS.

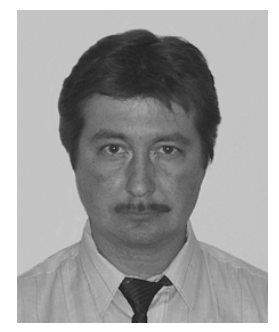

Sergei S. Orlov received the M.S. degree in physics and applied mathematics from Moscow Institute of Physics and Technology in 1991 and the the M.S.E.E. and Ph.D. degrees in electrical engineering from the California Institute of Technology, Pasadena, in 1993 and 1996, respectively.

From 1996 till 1999, he was a Senior Research Scientist at Optitek, Inc. and Siros Technologies, working on development of holographic data storage systems (HDSSs) and advanced holographic recording materials within the Defense Advanced Research Projects Agency HDSS and photorefractive information storage media (PRISM) programs. He is currently a Senior Research Scientist in the Solid State Photonics Lab of Electrical Engineering Department at Stanford University, Palo Alto, CA. He has published over 30 scientific articles, contributed several book chapters, and holds several patents in the fields of optical design, holographic recording materials, and volumetric optical storage. His research interests include holographic data storage, ferroelectric and photorefractive materials, volumetric optical storage materials, optical phase conjugation, and interaction of laser light with matter.

Dr. Orlov is a Member of the Optical Society of America. In 2001, he received a technical achievement award from National Storage Industry Consortium (INSIC) for "outstanding contributions to the development of holographic storage technology and to the NSIC HDSS and PRISM Research programs."

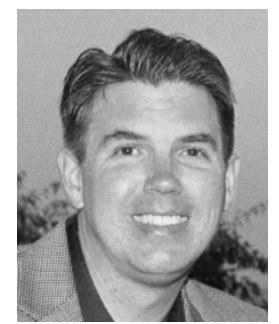

Matthew C. Bashaw received the B.S. degree in physics from the University of Notre Dame, South Bend, IN, in 1985 and the M.S., M.Phil., and $\mathrm{Ph} . \mathrm{D}$. degrees in engineering and applied science from Yale University, New Haven, CT, in 1987, 1988, and 1991, respectively.

He served as a Research Scientist at Stanford University, where he led several research teams focused on holography, nonlinear optics, optical signal processing, optical encryption, and other optical technologies. His team accomplishments include the first reported end-to-end digital holographic data storage system. He contributed significantly to the Stanford University Center for Nonlinear Optical Materials, the Photorefractive Information Storage Materials Consortium (PRISM), and the Holographic Data Storage Systems Consortium (HDSS), which included Defense Advanced Research Projects Agency sponsorship and industry collaboration. After Stanford, he held key positions at several startup companies developing leading-edge enabling technologies for information system products. His roles included research and development, technology transfer, product development, business development, and intellectual property strategy. In these capacities, he provided critical leadership in fiber optic networking, optical data storage, photonics technology, speech recognition tools, and online media distribution technology. He is currently a Senior Staff Engineer at Lockheed Martin Corporation. He is also a registered patent agent. He has authored or coauthored over 100 papers and formal presentations, including 32 peer-reviewed papers cited over 900 times. He holds nine patents and has managed company patent portfolios as an intellectual property strategist. 